\title{
Advanced Curation of Astromaterials for Planetary Science
}

\author{
Francis M. McCubbin ${ }^{1}$ (D) Christopher D.K. Herd ${ }^{2}$. Toru Yada ${ }^{3}$ Aurore Hutzler ${ }^{1}$. \\ Michael J. Calaway ${ }^{4}$. Judith H. Allton ${ }^{1}$ - Cari M. Corrigan ${ }^{5}$ - Marc D. Fries ${ }^{1}$. \\ Andrea D. Harrington ${ }^{1}$ - Timothy J. McCoy ${ }^{5}$ Julie L. Mitchell ${ }^{1} \cdot$ Aaron B. Regberg $^{1}$. \\ Kevin Righter ${ }^{1}$. Christopher J. Snead ${ }^{6} \cdot$ Kimberly T. Tait $^{7} \cdot$ Michael E. Zolensky $^{1}$. \\ Ryan A. Zeigler ${ }^{1}$
}

Received: 11 May 2019 / Accepted: 17 October 2019 / Published online: 7 November 2019

(C) The Author(s) 2019

\begin{abstract}
Just as geological samples from Earth record the natural history of our planet, astromaterials hold the natural history of our solar system and beyond. Astromaterials acquisition and curation practices have direct consequences on the contamination levels of astromaterials and hence the types of questions that can be answered about our solar system and the degree of precision that can be expected of those answers. Advanced curation was developed as a cross-disciplinary field to improve curation and acquisition practices in existing astromaterials collections and for future sample return activities, including meteorite and cosmic dust samples that are collected on Earth. These goals are accomplished through research and development of new innovative technologies and techniques for sample collection, handling, characterization, analysis, and curation of astromaterials. In this contribution, we discuss five broad topics in advanced curation that are critical to improving sample acquisition and curation practices, including (1) best practices for monitoring and testing of curation infrastructure for inorganic, organic, and biological contamination; (2) requirements for storage, processing, and sample handling capabilities for future sample
\end{abstract}

Role of Sample Return in Addressing Major Questions in Planetary Sciences

Edited by Mahesh Anand, Sara Russell, Yangting Lin, Meenakshi Wadhwa, Kuljeet Kaur Marhas and Shogo Tachibana

\section{F.M. McCubbin}

1 NASA Johnson Space Center, Mailcode XI2, 2101 NASA Parkway, Houston, TX 77058, USA

2 Department of Earth and Atmospheric Sciences, University of Alberta, 1-26 Earth Sciences Building, Edmonton, Alberta, T6G 2E3, Canada

3 JAXA, Sagamihara, 252-5210, Japan

4 Jacobs, NASA Johnson Space Center, Mail Code XI2/JETS, 2101 NASA Parkway, Houston, TX 77058, USA

5 Department of Mineral Sciences, National Museum of Natural History, Smithsonian Institution, Washington, DC, USA

6 Texas State University - Jacobs JETS Contract, NASA Johnson Space Center, Mail Code XI2/JETS, 2101 NASA Parkway, Houston, TX 77058, USA

7 Department of Natural History, Royal Ontario Museum, 100 Queen's Park, Toronto, Ontario M5S 2C6, Canada 
return missions, along with recent progress in these areas; (3) advancements and improvements in astromaterials acquisition capabilities on Earth (i.e., the collection of meteorites and cosmic dust); (4) the importance of contamination knowledge strategies for maximizing the science returns of sample-return missions; and (5) best practices and emerging capabilities for the basic characterization and preliminary examination of astromaterials. The primary result of advanced curation research is to both reduce and quantify contamination of astromaterials and preserve the scientific integrity of all samples from mission inception to secure delivery of samples to Earth-based laboratories for in-depth scientific analysis. Advanced curation serves as an important science-enabling activity, and the collective lessons learned from previous spacecraft missions and the results of advanced curation research will work in tandem to feed forward into better spacecraft designs and enable more stringent requirements for future sample return missions and Earth-based sample acquisition.

\section{Introduction}

Human fascination with the night sky and with celestial objects that fall to the Earth from the sky is as old as our species, and use of these astromaterials as a natural resource occurred at least as early as the Bronze Age (Jambon 2017; McCoy 2018; McCoy et al. 2017). However, the initial curation of astromaterials as objects of scientific interest to understand our universe began more recently (Marvin 2006) and in earnest with the curation of meteorite samples in museums starting in the year 1748 at the Natural History Museum Vienna (Brandstätter 2006). Meteorites have remained objects of fascination by scientists and the public alike with the establishment of many meteorite collections across the world. Meteorite recovery and curation practices vary widely and are highly dependent on many factors, including the knowledge and resources of the finder and the financial and technical support available for the collection in which the sample is curated. The scientific importance of the sample can also be a determining factor, but this is predicated on the aforementioned factors. All meteorites, regardless of how they were handled from recovery to curation, have experienced uncontrolled entry and exposure to the terrestrial environment, including, at minimum, the terrestrial atmosphere and the ground. This exposure results in terrestrial contamination, the amount of which is typically dependent on the physicochemical properties of the meteorite, the conditions at the fall site, and the amount of exposure time to the terrestrial environment. Consideration of these factors can also be determining factors in how a meteorite sample is curated. An overview of meteorite collections, their contents, and curation practices is available in McCall et al. (2006).

Until the 1960's, delivery of all astromaterials to Earth were unplanned events that required reactionary responses for recovery and curation. However, with the initiation of the Apollo program, direct return of pristine astromaterials from another body became possible, and with it, established the need to design a facility to keep those samples in a pristine state for an indefinite period of time. Planning for the Lunar Receiving Laboratory (LRL) began in 1964, and the facility was completed in 1967 (Calaway et al. 2017; McLane et al. 1967). As part of this planning, stringent protocols in the handling, storage, and processing of samples were developed. These protocols ensured that portions of the samples remained pristine or as close to an "as returned" state as possible in perpetuity to enable future scientific discoveries from the returned samples. The delivery of Apollo 11 samples to Earth occurred on July 24, 1969 at 12:50 EDT, four days after the first successful human landing on the Moon. This round-trip journey marked a transformative milestone in 
Table 1 Planetary sample return missions

\begin{tabular}{|c|c|c|c|}
\hline Program/Mission & Returned to Earth & Destination & $\begin{array}{l}\text { Returned } \\
\text { sample }\end{array}$ \\
\hline Apollo 11 (USA, NASA) & July 24, 1969 & Moon: Mare Tranquillitatis & $21.55 \mathrm{~kg}$ \\
\hline Apollo 12 (USA, NASA) & November 24, 1969 & Moon: Oceanus Procellarum & $34.30 \mathrm{~kg}$ \\
\hline Luna 16 (USSR) & September 24, 1970 & Moon: Mare Fecunditatis & $101 \mathrm{~g}$ \\
\hline Apollo 14 (USA, NASA) & February 9, 1971 & Moon: Fra Mauro Highlands & $42.80 \mathrm{~kg}$ \\
\hline Apollo 15 (USA, NASA) & August 7, 1971 & Moon: Hadley-Apennine & $76.70 \mathrm{~kg}$ \\
\hline Luna 20 (USSR) & February 25, 1972 & Moon: Apollonius Highlands & $30 \mathrm{~g}$ \\
\hline Apollo 16 (USA, NASA) & April 27, 1972 & Moon: Descartes Highlands & $95.20 \mathrm{~kg}$ \\
\hline Luna 24 (USSR) & August 22, 1976 & Moon: Mare Crisium & $170.1 \mathrm{~g}$ \\
\hline Apollo 17 (USA, NASA) & December 19, 1972 & Moon: Taurus-Littrow & $110.40 \mathrm{~kg}$ \\
\hline Genesis (USA, NASA) & September 8, 2004 & Earth-Sun Lagrange 1 & $\begin{array}{l}\text { Implanted Solar } \\
\text { Wind Atoms }\end{array}$ \\
\hline Stardust (USA, NASA) & January 15, 2006 & Comet Wild 2/Interstellar & $\begin{array}{l}\text { Small Particles } \\
\text { Captured in } \\
\text { Aerogel }\end{array}$ \\
\hline Hayabusa (Japan, JAXA) & June 13, 2010 & Asteroid 25143 Itokawa & $\begin{array}{l}\text { Tens of } \\
\text { thousands of } \\
\text { recovered small } \\
\text { particles }\end{array}$ \\
\hline Hayabusa2 (Japan, JAXA) & $\begin{array}{l}\text { December } 2020 \\
\text { (Planned; in Flight) }\end{array}$ & Asteroid 162173 Ryugu & $\begin{array}{l}0.1 \text { to } 10 \mathrm{~g} \\
\text { Planned }\end{array}$ \\
\hline OSIRIS-REx (USA, NASA) & $\begin{array}{l}\text { September } 2023 \\
\text { (Planned; in Flight) }\end{array}$ & Asteroid 101955 Bennu & $\begin{array}{l}0.06-2 \mathrm{~kg} \\
\text { Planned }\end{array}$ \\
\hline
\end{tabular}

human history and as the first sample return mission, provided the initial fuel to drive the burgeoning field of planetary sample science.

The planning process for curation prior to the return of the Apollo 11 samples set the precedent that curation involvement and planning begins at the inception of a sample return mission, and this founding principle has guided sample return missions subsequent to Apollo (e.g., Allen et al. 2011; Yada et al. 2014). There have been a total of 13 successful sample return missions, including six manned Apollo missions from NASA, three unmanned lunar sample return missions from the Union of Soviet Socialist Republics (USSR), the NASA Long Duration Exposure Facility that exposed various materials to the low-Earth orbit environment for approximately 6 years, the NASA Genesis mission that returned solar wind from the Earth-Sun Lagrange point 1 (L1), the NASA Stardust mission that returned particles embedded in aerogel from the coma of Comet Wild 2 and from interstellar space, and JAXA's Hayabusa mission that returned material from the surface of asteroid Itokawa (Table 1). In addition, there are two sample return missions in flight, including JAXA's Hayabusa2 mission that will return samples from the asteroid Ryugu and NASA's OSIRIS-REx mission that will return material from the asteroid Bennu (Table 1). Further details about each of these missions are provided in Table 1. With each successive sample return mission comes with it an important set of lessons learned that are used to inform subsequent sample return missions, and these lessons learned extend to curation standards and practices.

The Apollo program offered the first set of lessons learned and set forth the modern era of curation practices for astromaterials from the solar system. With the exception of USSR Luna missions, all sample return missions in the last two decades have built upon the 
legacy of Apollo. While recent missions have contributed to lessons learned, the majority of lessons learned and established practices can be linked to Apollo. The Apollo program actively sought out a wide range of scientists and eventually levied the scientific community at large to influence mission conception and design. Mission decisions and laboratory research on returned samples, at least peripherally, were focused substantially in maximizing science obtained from samples in laboratory research. The majority of these sample scientists were found in the field of geological sciences. The management environment was an integration of human spaceflight mission objectives, engineering constraints, sample scientists, and those responsible to prevent back contamination of the Earth. In this management structure, conflicts routinely arose and were not only turf battles, but were rooted in basic technical conflicts to balance crew safety, lunar sample preservation, and potential hazard containment for unknown biological pathogens. Since Apollo was a series of missions, it was possible to improve sampling hardware and laboratory handling devices using experience and samples from the lunar surface. For example, regolith drive tube function was greatly improved through redesign for Apollo missions 15-17 to allow deep penetration with minimal distortion of stratigraphy. Knowledge gained from examination of the first samples (Apollo missions 11-14) allowed the switch from a high-vacuum gloved handling environment to pure gaseous nitrogen positive pressure gloveboxes, which better preserved sample cleanliness and ease of use. A mission series like Apollo allows fine tuning of sample collection and returned sample handling as knowledge is acquired. Building upon Apollo and later sample return missions, a series of lessons learned and best practices for future sample return missions were developed and listed as follows:

- World-class scientific expertise: Integration of planetary sample scientists as advisors on science issues through formal organizations such as the historical Lunar Sample Analysis Planning Team (LSAPT) and Lunar and Planetary Science Team (LAPST) and as well as today's Curation and Analysis Planning Team for Extraterrestrial Materials (CAPTEM). CAPTEM currently presents findings to NASA on sample allocations ensuring best science and fair access to samples, current curation facilities, and inspection of laboratory operations, capabilities, capacity needs, and staffing. CAPTEM also provides findings for publicizing sample characterization information and service to the community. In addition, CAPTEM provides NASA with findings on design review of sample receiving and curation facilities as well as material restrictions/suggestions to preserve science value of samples.

- The integration of planetary science and geology training for astronauts, mission managers, and engineers involved in sample return missions.

- The integration of sample scientists into landing site selection, traverse planning, and sample acquisition.

- The integration of sample scientists into mission control operations and advisors during missions.

- The integration of Earth receiving and curation operations personnel into mission conception and engineering spacecraft design is critical for any sample return mission.

- Selection of materials that have low to zero particulate shedding mechanical properties for spacecraft, primary sample containment, handling, and storage equipment to preserve sample integrity.

- Selection of materials that have low to zero outgassing mechanical properties for spacecraft, primary sample containment, handling, and storage equipment to preserve sample integrity. 
- Selection of a diversity of materials for primary sample containment, handling, and storage equipment to enable scientific investigations of the entire periodic table, organic compounds, and biological matter.

- Sample return missions should establish a concept of sample segregation for primary mission goals (e.g., segregation of samples in different containment/isolation used for inorganic, organic, and biological investigations as well as focused goals of the mission). Sample acquisition and containment must always focus on prohibiting cross-contamination and preservation of the scientific integrity of each sample.

- The integration of curation, proper material selection, and cleaning into mission contamination control requirements and implementation during Assembly, Test, and Launch Operations (ATLO) is critical for sample return.

- Use of inert and/or vacuum environments or environments close to native collection environments for processing and storage of astromaterials. Develop standard practices to mitigate contamination from terrestrial atmosphere, pressures, and temperatures.

- Use of environmental monitoring methods, cleanroom technology, and biological safety isolation to maintain desired processing and storage environments.

Lessons learned not only inform our best practices, but they also help to identify strategic knowledge gaps that require new research to fill. Furthermore, if we look only at improving upon our current curation capabilities, we will not be prepared when returned samples require care that is very different from those within our current collections. At present, most returned samples are geological in nature, with the exception of the Genesis solar wind atoms that are implanted within a number of high purity material substrates. Most of the samples are kept close to room temperature and, when kept in the pristine environments of a clean laboratory, will maintain their fidelity indefinitely. However, future sample return missions could bring back samples that require storage and handling conditions outside of current capabilities, including gases, liquids, ices, or biological materials. To successfully curate these sensitive materials also requires new research, and we describe here a field of research that we refer to as advanced curation.

Advanced Curation is a cross-disciplinary field that seeks to improve curation practices in existing astromaterials collections, including meteorite and cosmic dust samples that are collected on Earth. Specifically, advanced curation has two primary goals that include (1) expansion of the sample processing and storage capabilities of astromaterials facilities to prepare for future sample return missions and Earth-based collection of astromaterials and (2) to maximize the science returns of existing astromaterials sample collections. These goals are accomplished through research and development of new innovative technologies and techniques for sample collection, handling, characterization, analysis, and curation of astromaterials. In addition, advanced curation includes testing and evaluation of new technologies and operational procedures for future sample return missions through human and robotic analog studies. Here we outline best practices and procedures and highlight new results, capabilities, and ongoing activities in the field of advanced curation of astromaterials. In particular, we outline (1) the best practices for monitoring and testing of curation infrastructure for contamination, (2) the development of new storage, processing, and sample handling capabilities, (3) the development and improvement of new astromaterials acquisition capabilities on Earth (i.e., the collection of meteorites and cosmic dust), (4) the importance of contamination knowledge strategies for maximizing the science returns of sample-return missions, (5) best practices and emerging capabilities for the preliminary examination and initial characterization of astromaterials, and finally (6) a summary of the biggest challenges that lie ahead as we look toward future sample-return initiatives. 


\section{Monitoring and Testing of Curation Infrastructure}

All sample return curation facilities are designed and built to meet specific controlled environment and cleanliness standards for the curated samples. Curation infrastructure is defined as all engineering systems that control the sample's storage and processing environment. This definition incorporates brick and mortar, temporary, modular, and mobile facilities. In addition, specialized equipment is included such as isolation chambers, gloveboxes, and desiccators that have the ability to alter the atmospheric chemistry, temperature, and pressure of the environment. During the Apollo program, curation infrastructure borrowed many innovative technologies from handling radioactive materials and biological quarantine practices. Today, curation infrastructure is derived from many industries including the nuclear, biotechnology, pharmaceutical, and semiconductor industries (USP 2013; Whyte 2001; Ramstorp 2000). Methods and techniques are either borrowed, augmented, or invented to maintain the controlled environment to mitigate terrestrial cross-contamination. Contamination covers any element that could compromise sample integrity. To quote the definition of pristine from Dworkin et al. (2018), it means that "no foreign material is introduced to the sample in an amount that hampers the ability to analyze the chemistry and mineralogy of the sample". While sample return missions designate contamination limits on specific elements and compounds at time of launch with focused science goals, samples are effectively allocated over time to study everything on the periodic table. Therefore, the implementation of curation infrastructure should be mindful that everything could be a contaminant to some research group. Modern cleanroom facilities have substantial infrastructure footprints that require continual monitoring to ensure they operate within the defined strict contamination control guidelines. This requires continuous monitoring and testing of the labs to verify that the sample processing environments remain clean from the standpoint of inorganic, organic, and biological contamination. As it is unrealistic to eliminate all contamination, careful monitoring and contamination knowledge must be conducted. To this end, curation laboratories that house astromaterials have developed numerous protocols and methods to monitor curation facilities and we outline those practices below.

\subsection{Real-Time Continuous Monitoring and Testing of Curation Cleanroom Laboratories}

Cleanrooms are a specialized controlled environment that must be continually monitored to verify whether they are working to defined parameters and specifications. The international standards organization (ISO) have developed fundamental standards for cleanrooms, namely ISO 14644. Curation cleanroom laboratories follow this standard as well as many adopted recommend practices from several industries (e.g., IEST, SEMI, GSA, etc.). For curation facilities, cleanroom measurements are regularly made to ensure that the heating ventilation, and air conditioning (HVAC) system is creating the appropriate cascade of positive or negative pressure and that Fan Filter Units (FFU), in conjunction with the HVAC system, are delivering the proper level of airborne particles to accepted limits for the planned ISO class. Temperature and humidity are also kept within pre-specified limits within the intended operational parameters of the HVAC system.

Ideally, real-time remote monitoring can track airborne particulates, room-to-room differential pressures, temperature, humidity, and HVAC operations. Remote airborne particle counters have either internal or external pumps with a flow rate of 0.1 CFM (2.83 LPM) or 1.0 CFM (28.3 LPM) dependent on ISO Class and desired statistics. Many of them can output up to 6 channels of simultaneous data within the range of $0.3-25.0 \mu \mathrm{m}$. For ISO 
Class 4 and below, a dedicated $0.1 \mu \mathrm{m}$ particle counter is desired to improve particle count statistics. While real-time remote monitoring is ideal, hand-held manual particle counters are sometimes used for spot checking spaces and annual ISO Class audits. For ISO Class 5 and above, these handheld particle counting instruments are typically set-up for a 2 minute measurement with a total sampling volume of $5.68 \mathrm{~L}$ and particle channels set at $0.3,0.5$, $0.7,1.0,5.0$, and $10.0 \mu \mathrm{m}$. In lieu of real-time continuous remote monitoring, weekly particle counts of all curation labs are desirable of key areas with a full ISO audits conducted annually or bi-annually.

Curation cleanroom laboratories primarily use a positive pressure differential barrier to reduce contamination. A pressure differential barrier is based on the concept of using positive pressure air flow cascade to create a cleaner zone towards a less clean zone as a first line of defense to prevent cross-contamination between two adjacent spaces. The pressure differential should be of significant magnitude and stability to prevent any reversal of air flow between barriers including when barrier thresholds are crossed and/or doors are opened. However, the pressure differential should not be too high as to create turbulent air flow that could compromise the clean zone. In addition, too high of pressure between zones can also prevent doors from opening. For example, at $0.10 \mathrm{inH}_{2} \mathrm{O}$ (inches of water), a $3 \times 7 \mathrm{ft}$. door requires $11 \mathrm{lbs}$. of force to open and close. Furthermore, this pressure results in unwanted turbulent air flow. ISO 14644-4, the design, construction, and start-up of cleanrooms and associated controlled environment, contains the international standard for cleanroom air-flow monitoring. ISO 14644-4 Section A.5.3 states that the pressure between clean zones should be set at: $\Delta P=0.02$ to 0.08 inH $_{2} \mathrm{O}$ (5 to $20 \mathrm{~Pa}$ ).

The cleanroom technology literature generally recommends a pressure differential of $0.04 \mathrm{inH}_{2} \mathrm{O}(10 \mathrm{~Pa})$ between two cleanrooms and a pressure differential of $0.06 \mathrm{inH}_{2} \mathrm{O}$ (15 Pa) between the cleanroom and an unclassified room (Sakraida 2008; Whyte 2001). Whyte (2010) discusses the reason for ISO 14644-4 acceptable minimum of $0.02 \mathrm{inH}_{2} \mathrm{O}$ ( $5 \mathrm{~Pa}$ ) pressure between adjacent rooms. This acceptable minimum was established for processing facilities that handle products that can be adversely affected from greater pressures. These low pressure differentials can sometimes be found in long tunnels between processing cleanrooms that contain air flow sensitive products. Whyte (2010) further discusses if 0.02 $\mathrm{inH}_{2} \mathrm{O}(5 \mathrm{~Pa})$ must be used; confirmation of the air flow direction must be verifiable with routine observable smoke flow tests (assuming such tests would not be a source of contamination). Sakraida (2008) discusses recent experimental studies that have tested the optimal pressure differential between clean zones. Pressure differentials between 0.03 to $0.05 \mathrm{inH}_{2} \mathrm{O}$ were determined to be optimal for mitigating cross-contamination. The study further suggested that clean zones with pressures above $0.05 \mathrm{inH}_{2} \mathrm{O}$ showed little increased benefit to mitigate contamination compared to increased energy costs of operating the air handling unit.

Based on ISO 14644-4 standards and available cleanroom technology literature, astromaterials curation laboratories should ideally maintain $\geq 0.05 \mathrm{inH}_{2} \mathrm{O}$ between interior "dirty" hallways to laboratory anterooms and a minimum of 0.03 to $0.05 \mathrm{inH}_{2} \mathrm{O}$ in most adjacent rooms between anteroom and main laboratory. For primary astromaterials storage areas and processing laboratories, ideally 0.05 to $0.08 \mathrm{inH}_{2} \mathrm{O}$ should be maintained to mitigate the long-term infiltration of contaminates. However, it is important to note that higher pressures may be desired to create a buffer to mitigate the risk of dropping below $0.05 \mathrm{inH}_{2} \mathrm{O}$ based on air flow stability from the HVAC and laboratory layout.

Real-time continuous remote monitoring is common for modern cleanrooms with a desired differential pressure accuracy of about $\pm 0.001 \mathrm{inH}_{2} \mathrm{O}$ or better. For older cleanroom laboratories, manual magnehelic differential pressure gauges are sometimes still used for 
monitoring differential pressures. In addition, annual or biannual differential pressure audits are conducted between each room doorway threshold with a handheld manometer and data placed on a building map to verify proper cascade of pressures.

HVAC ON/OFF and velocity $(\mathrm{m} / \mathrm{s})$ are continually monitored in real-time. The data displayed are also used to check air changes per hour towards the as-built of the cleanroom and ISO standards. The FFUs are biannually or annually checked to be running at $90 \pm$ $10 \mathrm{fpm}$. While FFUs are typically not monitored in real-time, this is an important routine check to assess failing blower motors and the efficiency of the ULPA or HEPA filters to determine when they need to be replaced.

Electrostatic charging and discharging in curation laboratories has the potential to cause damage to samples and equipment. In addition, electrostatic discharges are a serious safety hazard to laboratory personnel. Most curation cleanrooms maintain a temperature between 24 to $15{ }^{\circ} \mathrm{C} \pm 1.0{ }^{\circ} \mathrm{C}$ and relative humidity (RH\%) of $<65 \%$ to $>35 \% \pm 1.0 \% \mathrm{RH}$. These ranges are based on ISO 14644-4 and ISO 14644-5 standards and are only for laboratory environments and do not reflect the environment of containment, such as in gloveboxes where moisture $\left(\mathrm{H}_{2} \mathrm{O}\right)$ is commonly measured below $1 \mathrm{ppm}$.

A deviation in any of these parameters or over a certain threshold (per curation protocol specific to the collection) triggers an investigation to understand the source of the problem and mitigate any faults. In case the issue cannot be resolved in a timely manner, samples are securely placed into storage and work stops in the lab, especially for samples processed outside of gloveboxes.

\subsection{Real-Time Continuous Monitoring of Curation Infrastructure Systems}

\subsubsection{Inert Environments}

Most pristine astromaterials benefit from not being stored and processed in terrestrial atmosphere. Since Earth's atmosphere is an oxidizing environment, preservation of astromaterials are preferred to be placed in an indigenous, vacuum, or inert environment. Most astromaterials on Earth are stored and processed in an inert gas such as nitrogen, argon, or helium, with the exception of JAXA's vacuum receiving glovebox used for the Hayabusa mission. Of these three inert gases, nitrogen is the most cost effective and is often chosen over argon and helium for routine storage. However, nitrogen analysis of astromaterials samples are compromised by processing in nitrogen, so nitrogen is not used exclusively.

At NASA Johnson Space Center (JSC), building 31 and $31 \mathrm{~N}$ has a dedicated 15000 gallon liquid nitrogen $\left(\mathrm{LN}_{2}\right)$ tank and tank farm that converts high purity $\mathrm{LN}_{2}$ to gaseous nitrogen $\left(\mathrm{GN}_{2}\right)$ for the entire building infrastructure. This nitrogen gas system provides an inert environment for processing and storing all NASA extraterrestrial sample collections where gloveboxes and desiccators consume $\sim 3500 \mathrm{scfh}$ of $\mathrm{GN}_{2}$. After gas production, the $\mathrm{GN}_{2}$ is filtered for particulates by the use of sintered 316 stainless steel filters ( 1 micron filtration at the tank farm and $3 \mathrm{~nm}$ point-of-use filters connected to all devices). In addition to $3 \mathrm{~nm}$ particulate filtration, the Genesis lab uses point of use Pall gas purifiers that reduces any $\mathrm{H}_{2} \mathrm{O}, \mathrm{CO}_{2}, \mathrm{O}_{2}$, and $\mathrm{CO}$ in the $\mathrm{GN}_{2}$ to $<1 \mathrm{ppb}$. The $\mathrm{LN}_{2}$ is a modified Grade $\mathrm{C}$ per MILPRF-27401G [ $\left[\mathrm{LN}_{2}\right.$ purity $99.995 \% ; \mathrm{H}_{2} \mathrm{O}<10$ ppm; Total Hydrocarbons as $\mathrm{CH}_{4}<1.0 \mathrm{ppm}$; $\mathrm{O}_{2}<10$ ppm; $\mathrm{H}_{2}<10$ ppm; $\mathrm{Ar}<20$ ppm; $\mathrm{CO}_{2}<10$ ppm; $\mathrm{CO}<10$ ppm; and particulates $<1.0 \mathrm{mg} / \mathrm{L}] . \mathrm{LN}_{2}$ is delivered to JSC weekly and the Curation Office periodically tests the purity of the liquid nitrogen beyond the NASA contract audits. For periodic sampling of the $\mathrm{LN}_{2}$, a cryogenic liquid sampler is connected directly to the $\mathrm{LN}_{2}$ tanker truck with the sampler hose. The $\mathrm{LN}_{2}$ sample is taken to an outside laboratory for analysis. The boil-off 
of this $\mathrm{LN}_{2}$ at the tank farm produces high purity gaseous nitrogen $\left(\mathrm{GN}_{2}\right)$. JSC currently tests the purity of the delivered $\mathrm{GN}_{2}$ by conducting airborne molecular organic sampling and SEM triage of inorganic particulates captured in $3 \mathrm{~nm}$ sintered stainless steel filters. Adsorbent sample tubes are used for sample collection and sent outside to Balazs Nanoanalysis for TD-GC-MS analysis. $\mathrm{GN}_{2}$ results routinely show no infiltration particulates past the filters and organic compounds and all hydrocarbon loads are below the reporting limit of $<0.1 \mathrm{ng} / \mathrm{L}$ for $>\mathrm{C} 7$. The $\mathrm{GN}_{2}$ is also tested monthly for the nitrogen isotopic ratio in a Finnigan MAT 253 IR-MS to ensure that no fractionation occurs over time or within the line. K-bottles of $\mathrm{GN}_{2}$, Ar, and $\mathrm{He}$ are also supplied at high purity research grade when required for certain processing activities or experiments. For example, the Subzero Facility for Curation of Astromaterials at the University of Alberta (see Cold Curation section) uses high-purity (99.998\%) Ar as a source, which is then further refined using a purification system to bring oxygen $\left(\mathrm{O}_{2}\right)$ and moisture $\left(\mathrm{H}_{2} \mathrm{O}\right)$ levels to $<0.1$ ppmv (Herd et al. 2016).

\subsubsection{High Purity Cleaning Agents}

Cleaning curation sample handling tools, containers, and other equipment (such as gloveboxes, isolation chambers, and desiccators) is required for the curation of astromaterials. Precision cleaning is typically required where equipment is cleaned to a specified cleanliness and the cleanliness is measured and verified to a standard. These precision cleaning facilities are not a small foot-print and use substantial consumables and equipment for operations. During final precision cleaning, specialized equipment is needed to purify the aqueous cleaning solutions. Historically, Apollo used Freon 113 as the final cleaning agent. The Freon 113 recycled in-house by distillation to achieve the required high purity. Today, NASA JSC uses ultrapure water (UPW) as the final cleaning agent and requires substantial initial investment ( $>$ \$3M USD) and monthly maintenance cost. For JSC, UPW is not only used for precision cleaning, but is also used to decontaminate Genesis solar wind materials contaminated by macro particles during the hard landing (see Genesis section).

The UPW purity is maintained and monitored in continuously flowing production lines. The JSC UPW plant produces 10 gallons/minute of UPW serving 5 laboratories throughout the building within a continuous flowing final loop connected to a 1000 gallon supply tank. Future upgrades to the system will increase the capacity to a 5000 gallon tank producing 15 gallons/minute serving 7 laboratories. Once UPW leaves the final flowing loop, within $<5$ seconds, $\mathrm{CO}_{2}$ and other compounds in the air quickly dissolve into the highly deionized water and resistivity is immediately lowered from $\sim 18.18 \mathrm{M} \Omega-\mathrm{cm}$ to $<1.0 \mathrm{M} \Omega$-cm. Therefore, UPW cannot be stored or transported in containers for use and UPW must be used directly from the flowing final loop for the maximum cleaning effectiveness. The UPW system is outfitted with a continuous real-time monitoring of critical components of the system as well as final water quality. The system monitors flow rate, pressure, resistivity, conductivity, temperature, particulates, total organic carbon, and tank levels. The UPW system conforms to ASTM D 5127-13, Standard Guide for Ultra-Pure Water Used in the Electronics and Semiconductor Industries and produces E-1.1 or better quality of water with a resistivity of $18.18 \mathrm{M} \Omega-\mathrm{cm}$ and total organic carbon (TOC) between 1 to $3 \mathrm{ppb}$. The quality of the water is routinely tested at least once a year or more for the following: (1) Anions by IC ranging from $>0.05$ to $0.02 \mathrm{ppb}(\mu \mathrm{g} / \mathrm{L})$ of Fluoride $\left(\mathrm{F}^{-}\right)$, Chloride $\left(\mathrm{Cl}^{-}\right)$, Nitrite $\left(\mathrm{NO}^{2-}\right)$, Bromide $\left(\mathrm{Br}^{-}\right)$, Nitrate $\left(\mathrm{NO}^{3-}\right)$, Phosphate $\left(\mathrm{HPO}_{4}^{2-}\right)$, and Sulfate $\left(\mathrm{SO}_{4}^{2-}\right)$; (2) Monovalent \& Divalent Cations by IC ranging from $>0.02$ to $0.01 \mathrm{ppb}(\mu \mathrm{g} / \mathrm{L})$ of Lithium $\left(\mathrm{Li}^{+}\right)$, Sodium $\left(\mathrm{Na}^{+}\right)$, Ammonium $\left(\mathrm{NH}_{4}^{+}\right)$, Potassium $\left(\mathrm{K}^{+}\right)$, Magnesium $\left(\mathrm{Mg}^{2+}\right)$, and Calcium $\left(\mathrm{Ca}^{2+}\right)$; (3) 30 elements Ultra Low Level in UPW by ICP-MS ranging from $>10$ to $0.02 \mathrm{ppt}$ (ng/L) of Aluminum (Al), Antimony (Sb), Arsenic (As), Barium (Ba), Bismuth (Bi), Boron (B), Cadmium 
(Cd), Calcium (Ca), Chromium (Cr), Cobalt (Co), Copper (Cu), Gallium (Ga), Germanium (Ge), Iron (Fe), Lead (Pb), Lithium (Li), Magnesium (Mg), Manganese (Mn), Mercury (Hg), Molybdenum (Mo), Nickel (Ni), Potassium (K), Silver (Ag), Sodium (Na), Strontium (Sr), Tin (Sn), Titanium (Ti), Tungsten (W), Vanadium (V), and Zinc (Zn); (4) Low-level Dissolved Silica at $>0.1 \mathrm{ppb}(\mu \mathrm{g} / \mathrm{L})$; (5) Bacteria-ASTM Method-F1094—87 $48 \mathrm{Hr}$ Incubation reported in $>1$ Bacteria per $100 \mathrm{~mL}$ cfu.

\subsubsection{Gloveboxes and Desiccators}

The inert environments of gloveboxes and desiccators that house astromaterials in storage or during processing should also be monitored. These environments are typically monitored continuously in real-time for their pressure, temperature, and known contaminates. For Apollo lunar material stored and processed in inert $\mathrm{GN}_{2}$, as well as the Subzero Facility used for processing Tagish Lake and other pristine astromaterials (Herd et al. 2016), gloveboxes are continuously monitored for $\mathrm{O}_{2}$ and $\mathrm{H}_{2} \mathrm{O}$ at a resolution of $\pm 1 \mathrm{ppm}$. For the lunar sample collection at JSC, these environments are required to be at $1 \mathrm{inH}_{2} \mathrm{O}$ positive pressure, room temperature, $<25 \mathrm{ppm}$ of $\mathrm{O}_{2}$, and $<50 \mathrm{ppm}$ of $\mathrm{H}_{2} \mathrm{O}$; but actual achievable can be $<1.0$ ppmv for $\mathrm{H}_{2} \mathrm{O}$ and $<15 \mathrm{ppmv}$ for $\mathrm{O}_{2}$ with the current system. It should be noted that the vast majority of $\mathrm{H}_{2} \mathrm{O}$ and $\mathrm{O}_{2}$ levels in gloveboxes do not originate from the $\mathrm{GN}_{2}$ supply lines, but from the isolator gaskets and gloves through molecular infiltration of terrestrial atmosphere even under $1.0 \mathrm{inH}_{2} \mathrm{O}$ positive pressure.

\subsection{Inorganic and Organic Testing of Curation Clean Labs}

Since 1998, the NASA JSC Curation Office has contracted Air Liquide Balazs Nanoanalysis to analyze airborne molecular inorganic and organic contaminates in cleanrooms and laboratory suites (Calaway et al. 2014). Following sampling protocols developed for the semiconductor industry, vertical exposure of $8^{\prime \prime}$ and 6 " diameter high purity silicon semiconductor wafers are exposed for 24-hours on a work surface or inside gloveboxes to better understand the airborne molecular contamination (AMC). The AMC data is also used to calculate the rate of deposition of surface molecular contamination (SMC). The inorganic and organic AMC for cleanroom monitoring is reported using ISO 14644-8 Classification of Air Cleanliness by Chemical Contamination (ACC) and the SMC for ISO 14644-10 Classification of Surface Cleanliness by Chemical Concentration.

For routine inorganic lab and glovebox monitoring, pre-cleaned $8^{\prime \prime}$ silicon wafers are packaged in two separate polypropylene wafer carriers; one for sample exposure and one for control, which is not opened. After a 24 or 48 hour of vertical exposure, Vapor Phase Decomposition Inductively Coupled Plasma Mass Spectrometry (VPD ICP-MS) is conducted at Balazs laboratories in Freemont, CA. The VDP-ICP-MS analyses report 35 elements (Al, As, B, Ba, Be, Ca, Cd, Ce, Co, Cr, Cu, Fe, Ga, Ge, Hf, In, K, La, Li, Mg, Mn, Mo, Na, $\mathrm{Ni}, \mathrm{Pb}, \mathrm{Sb}, \mathrm{Sn}, \mathrm{Sr}, \mathrm{Ta}, \mathrm{Ti}, \mathrm{W}, \mathrm{V}, \mathrm{Y}, \mathrm{Zn}$, and $\mathrm{Zr}$ ) with reporting limits ranging from $10^{8}$ to $10^{10}$ atoms $/ \mathrm{cm}^{2}$.

For routine organic lab and glovebox monitoring, two sets of prebaked 8 " silicon wafers are sandwiched together and triple-wrapped in baked-out aluminum foil; two for sample exposure and two for control, which are not opened. After a 24 or 48 hour vertical exposure on an aluminum stand, Thermal Desorption Gas Chromatography Mass Spectroscopy (TDGC-MS) is conducted at Balazs laboratories. The TD-GC-MS measures organic compounds from C6 to $\mathrm{C} 28$ with a reporting limit of $0.1 \mathrm{ng} / \mathrm{cm}^{2}$. 
In addition to organic wafer exposure, which collect airborne molecular and particulate contaminants well, proprietary air absorbent tests are routinely conducted to better understand hydrocarbon and volatile organic compound (VOC) load in cleanroom air or glovebox gaseous nitrogen environments. This test is implemented with an adsorbent tube with a pump running at $100 \mathrm{~mL} / \mathrm{min}$, for 6 hours that is exposed to the cleanroom or glovebox. The adsorbent tube is analyzed using the same TD-GC-MS method as the organic wafer, but with a reporting limit of $0.1 \mathrm{ng} / \mathrm{L}$.

Besides these traditional methods of monitoring, the JSC Curation Office also employs the use of optical microscopy and Scanning Electron Microscopy (SEM) as a basic method of direct analysis for inorganic and organic contaminates for the cleanroom laboratory and infrastructure. Cleanroom construction materials, surfaces, sample handling tools, containers, and unknown visible material are analyzed directly or with tape-pulls or polyester wipes. Optical microcopy and SEM typically are used as an initial screening before using other methods of analysis. The following methods have been used in the past at the NASA JSC Curation office, on an as-needed basis, on witness plates, test coupons, millipore filters, and other material samples: (1) Optical Stereomicroscopy/Microscopy for macro particulate/other contamination, (2) FEG-SEM/EDX for micro particulate identification, (3) FT-IR and Raman Spectroscopy for surface contamination, (4) XPS for complete surface/thinfilms/oxidation, (5) LA-HR-ICP-MS for gross surface inorganics, (6) VPD-HR-ICP-MS for molecular airborne inorganics, (7) TD-GC-MS with GL Sciences SWA-256 wafer analyzer for molecular airborne organics/outgassing, (8) DART-qTOF-MS for gross surface organics, (9) LC-MS for amino acids, and (10) AFM (Atomic Force Microcopy) for surface roughness/thin-films/cleaning changes. Although not continuously monitored, the Subzero Facility for the Curation of Astromaterials used solid phase microextraction (SPME) fiber GC-MS methods to characterize the glovebox atmosphere during commissioning (Herd et al. 2016); this method shows potential for use in continuous monitoring, although its use requires the assessment and selection of appropriate SPME fibers for the airborne organic compounds of interest.

\subsection{Biological Testing of Curation Cleanlabs}

Biological testing of clean labs is important in many commercial and academic settings, and biological testing in aerospace and medical settings, like spacecraft assembly facilities, hospital cleanrooms, and pharmaceutical production labs are discussed here in the context of the best practices for monitoring astromaterials curation facilities. The monitoring methods differ among these labs, but the overall goal, to reduce or eliminate contamination, is always the same. A key difference for curation facilities is the need to identify contaminants. Identification is not always a monitoring plan requirement in other industries. Microorganisms like bacteria and fungi are capable of physically and chemically altering astromaterials (Toporski and Steele 2007). Since the nutrient levels in cleanrooms are purposely kept at very low levels, it is likely that microorganisms will seek out nutrient bearing phases in the astromaterials themselves (e.g. phosphorous rich minerals, organic carbon). Therefore, it is important to identify organisms in cleanrooms and understand how they might affect samples stored within the cleanrooms. The most common monitoring method for any cleanroom is cultivation of viable microorganisms like bacteria and fungi. The implementation of a variety of culture-independent analysis techniques that are employed more sporadically are also discussed.

In the aerospace industry, biological testing is most commonly performed to meet planetary protection requirements for individual pieces of hardware and entire missions. The 
goals are defined by Article IX of the 1967 United Nations Treaty on, "Principles Governing the Activities of States in the Exploration and Use of Outer Space, Including the Moon and Other Bodies". More detailed policies are outlined by COSPAR (Committee on Space Research) (COSPAR 2011). The sampling and testing methods are implemented by NASA (NASA 2010, 2017) and/or ESA (ECSS 2008).

NASA requirements for sampling an aerospace cleanroom to meet planetary protection requirements are described in the "Handbook for the Microbial Examination of Space Hardware" (NASA 2010). Briefly, samples are collected with sterile swabs or wipes made of cotton or preferably a synthetic material like polyester. The samples are exposed to $80{ }^{\circ} \mathrm{C}$ for 15 minutes and any surviving microorganisms are transferred to Petri dishes filled with Tryptic Soy Agar (TSA) and incubated at $32{ }^{\circ} \mathrm{C}$ for 72 hours. Cultured organisms are counted but not necessarily identified. ESA requirements are similar, but require cultivation on Reasoners 2 Agar (R2A) for oligotrophic bacteria, Thioglycolate Agar (TGA) for anaerobic bacteria, and Potato Dextrose Agar (PDA) for fungi in addition to TSA (ECSS 2008). Only one set of R2A plates are heat shocked, while the remaining samples are incubated without being exposed to heat. The ESA standards also include provisions for collecting air samples with an impactor style sampling device. Sampling to meet planetary protection requirements is conducted with the assumption that all of the hardware will be exposed to DHMR (dry heat microbial reduction) or an equivalent process to sterilize the spacecraft. Organisms that survive the heat-shock treatment are counted as a proxy for what might be capable of surviving DHMR. These are fit for purpose assays that are not designed or intended to capture the total diversity of the cleanroom environment. While some facilities and/or missions do identify and archive isolates, this is not required or routine in every instance. Similar culture-based assays have been used to monitor Chinese and Russian space craft assembly facilities as well (Novikova 2004; Zhang et al. 2018).

Cleanrooms used to manufacture pharmaceuticals and package food are also monitored for biological contamination. There are no detailed methods for how to monitor these types of cleanrooms, but cultivation-based techniques are generally the norm. ISO 14698-1 sets out very general principles and methods for biocontamination control in cleanrooms. The document states, "The appropriate sampling method and related procedures shall be selected and performed to reflect the complexity and variety of situations. Sampling shall be carried out using a device and method selected in accordance with the written procedure and in accordance with the instructions provided by the device manufacturer," (ISO14698 2003). The United States Pharmacopeial Convention also relies on cultivation based methods without specifying a particular set of sampling tools, growth conditions, or nutrients (USP 2013). For example, air samples can be collected with a variety of tools, including: slit to agar samplers, centrifugal samplers, gelatin filter samplers, sieve impactors, impingers, and settle plates (USP 2013). However, the USP document does make several important points regarding sampling methods and data analysis: (1) Total particulate counts from air sampling do not correlate to microbial abundance, although this is an area of open research (Raval et al. 2012). (2) Microbial monitoring is semi-quantitative at best. (3) Colony counts (i.e. the number of culturable organisms) are highly variable from sample to sample and from day to day. Recovery rate is a more reliable statistic for defining a microbial baseline. Recovery rate is defined as: $\frac{\# \text { of samples with }>0 \text { CFU }}{\text { \# total number of samples collected during a sampling event }}$ where CFU is a colony forming unit. For example, an aseptic ISO 7 cleanroom should have a baseline recovery rate $<10 \%$ (USP 2013). The USP document also emphasizes the importance of identifying cleanroom isolates and taking action when new isolates appear and or when an individual sample contains $>15 \mathrm{CFU}$. In general, sampling of pharmaceutical cleanrooms 
is focused on cultivating mesophilic organisms from surface swabs, air samples, and cleanroom personnel (Sandle 2012; Whyte 2010). A variety of media types and growth conditions are acceptable as long as they are suitable for enumerating the organisms of concern.

Standard efforts to monitor microbial contamination in cleanrooms rely on cultivation based techniques across all industries. Cultivation based techniques are relatively cheap and easy to perform on a regular basis. However, they can be highly variable and even the most comprehensive culture-based sampling campaign is guaranteed to under-sample the environment (e.g., Hug et al. 2016; Lynch and Neufeld 2015; Rappe and Giovannoni 2003). The community recognizes the need to assess these, "unculturable," organisms and has employed a variety of techniques to do so.

Next generation DNA sequencing is the most common culture-independent method. Amplification and sequencing of marker genes (tag or amplicon sequencing) like the ribosomal $16 \mathrm{~S}$ gene for bacteria and archaea and the ITS region for fungi is one promising method for monitoring unculturable organisms in the cleanroom environment. This measurement has changed as the sequencing platforms have improved. Initial tag sequencing was performed using clone libraries and Sanger type sequencing, which only generates about 1000 base pairs of data for a single organism at a time (Shokralla et al. 2012). The 454 platform generates $10^{2}-10^{4}$ sequences per sample and has allowed researchers to identify hundreds of OTU's (operational taxonomic unit) or organisms per sample (La Duc et al. 2014; MoisslEichinger et al. 2015; Vaishampayan et al. 2013). Using this technology, archaea were found to be persistent, viable (Moissl-Eichinger 2011) members of some cleanroom communities (Moissl-Eichinger 2011; Moissl-Eichinger et al. 2015; Moissl et al. 2008). The Illumina sequencing platforms are the current standard for tag sequencing (Mahnert et al. 2015; Minich et al. 2018; Mora et al. 2016). These sequencers can generate $10^{5}-10^{6}$ sequences per sample, allowing researchers to identify even more organisms. A recent tag sequencing survey of the SAF (spacecraft assembly facility) at JPL identified $>16000$ OTU's. Tag sequencing is a powerful monitoring tool, but it does have several important biases. PCR (polymerase chain reaction) based amplification of DNA is required for most low biomass samples. This amplification step does not amplify DNA from every organism equally. For example Moissl-Eichinger et al. (2015) were able to cultivate organisms that they did not detect using, "universal" PCR primers for amplification and subsequent tag sequencing. Secondly, sequencing of any type cannot distinguish DNA from viable organisms from relict environmental DNA inside dead organisms. Several researchers have started treating their samples with compounds like PMA (propidium monoazide) to destroy DNA from nonviable organisms prior to sequencing (e.g., Mahnert et al. 2015; Moissl-Eichinger et al. 2015; Mora et al. 2016; Weinmaier et al. 2015; Zhang et al. 2018). Due to variations in primer choice, sequence length, error rate, and total number of sequences produced, it is very difficult to quantitatively compare data generated by different sequencing platforms (Tremblay et al. 2015). Care should be taken to keep these variables as consistent as possible during monitoring. When changes are made, they should be directly compared to previous methods.

Rather than amplifying and sequencing specific marker genes, it is also possible to sequence all of the DNA in a sample (with or without amplification) using the same types of DNA sequencers discussed above. This sequencing technique is commonly referred to as shotgun metagenomics (e.g., Bashir et al. 2016; Minich et al. 2018; MoisslEichinger et al. 2015; Weinmaier et al. 2015). Shotgun metagenomics provides more information about the function of abundant organisms in the environment but often fails to detect rare members of the community (Tessler et al. 2017). Additionally, this technique generates large amounts of data that can be very challenging and time-consuming to interpret. At present, metagenomics is a powerful research tool, but it is probably not yet 
suitable for routine monitoring. New DNA sequencers, like the MinIon platform (Reuter et al. 2015), that generate longer reads may eventually be able to generate metagenomic data that are easier to assemble and interpret, but they are still being developed and improved.

DNA sequencing can be used to inform the design of more rapid assays for biological monitoring. qPCR (quantitative polymerase chain reaction) can be used to assess the number of copies of genes in a sample that directly correlates to microbial abundance (Cooper et al. 2011; Hubad and Lapanje 2013; Kwan et al. 2011; Mahnert et al. 2015; Moissl-Eichinger 2011; Moissl-Eichinger et al. 2015; Schwendner et al. 2013; Vaishampayan et al. 2013; Zhang et al. 2018). When interpreting qPCR data, care must be taken to account for organisms that have multiple copies of the $16 \mathrm{~S}$ or other marker gene (Větrovský and Baldrian 2013). DNA microarrays like the Phylochip have also been investigated as potential monitoring tools (Cooper et al. 2011; Jimenez 2011; La Duc et al. 2009, 2014; Probst et al. 2010; Vaishampayan et al. 2013). Both of these techniques show promise as monitoring solutions, but they probably require initial investigation with culturing and DNA sequencing in order to ensure that probes and primers are designed to capture the communities present inside the cleanroom in question.

Techniques that do not involve sequencing DNA are also being tested in cleanroom settings. All living organisms on Earth produce a compound called ATP (adenosine triphosphate) for energy storage. Measuring the concentration of ATP in a cleanroom sample provides information about the total number of viable cells (Benardini and Venkateswaran 2016; La Duc et al. 2007; Mahnert et al. 2015; Venkateswaran et al. 2003), but it is not useful for identifying what organisms are present. MALDI-TOF (Matrix assisted laser desorption time of flight) mass spectrometry is now commonly used in the medical field to identify organisms, and it is being applied in aerospace cleanrooms as well (Andrade et al. 2018; Moissl-Eichinger et al. 2015). However, this technique is still dependent on culturing organisms. Fluorescence based monitoring systems can detect airborne cells but cannot identify them (Hallworth 2012).

Biological testing of clean labs suffers from a lack of repetition. Outside the NASA standard assay, very few measurements are routinely replicated between labs. In some respects, this is good and appropriate. Monitoring methods should be modified to suit the environment and the questions being asked. The microbial profile of cleanrooms will be different in different environments (e.g., La Duc et al. 2009). For example, cold curation facilities should explicitly test for the presence of psychrophilic organisms (Sandle and Skinner 2013). It would be a waste of resources to look for psychrophiles in labs maintained at room temperature. However, variations in sample collection, DNA extraction, DNA sequencing, and data processing methods make inter-lab comparisons very difficult. Testing new methods and techniques is an important area of research, but more effort should be made to relate these new measurements to previously generated data.

Curation labs should design a monitoring plan that is capable of quantifying and identifying the microbes present therein. Unfortunately, there is no single measurement or technique capable of thoroughly describing a microbial community. Each method discussed above has its strengths and weaknesses. Therefore, a selection of culture-based and cultureindependent techniques should be used to monitor cleanroom ecology. Samples should be collected from the air and from surfaces regularly and as frequently as daily when critical operations are being conducted. Special care should be taken to avoid organic or inorganic contamination during sampling. For example, agar filled contact plates used in the pharmaceutical industry are inappropriate for curation labs since they would introduce bioavailable organic compounds and trace metals into the lab. Most importantly, sample collection and 
analysis methods should be as consistent as possible in order to generate a baseline dataset that can be used as a basis of comparison for new techniques. Regular and consistent sampling is the most important feature of any environmental monitoring program.

\section{Development of New Storage, Processing, and Sample Handling Capabilities}

As technological advancements and new ideas expand the variety and scope of scientific questions that can be asked with astromaterials samples, so expands the need for better storage, processing and sample handling capabilities of curation laboratories that house and process astromaterials samples. Here we summarize a number of important advancements and areas of growth in sample storage, processing, and handling techniques that will be important in the coming decades for maximizing science returns on astromaterials samples.

\subsection{Cold Curation of Astromaterials and Associated Gases, Biological Samples, and Hardware}

The ever-expanding plans for the return of samples from volatile-rich solar system targets and/or targets of astrobiological significance necessitates the development of curation at temperatures below that of typical curation facilities $\left(20^{\circ} \mathrm{C}\right)$. Temperature requirements depend primarily on which volatiles are expected within the returned sample, which in turn relate to the conditions under which the material formed and has since been preserved. The term "cryogenic" is defined as relating to temperatures below $-183{ }^{\circ} \mathrm{C}$; the normal boiling points of the noble gases, oxygen, nitrogen, and air lie below this temperature. More generally, "cryogenic" refers to temperatures below approximately $-150{ }^{\circ} \mathrm{C}$ (https://www.nist.gov/mml/acmd/cryogenics/aboutcryogenics). The term "high temperature cryogenic" is used to refer to temperatures from the boiling point of liquid nitrogen, $-196{ }^{\circ} \mathrm{C}$, up to $-50{ }^{\circ} \mathrm{C}$, the generally defined limit of cryogenics (e.g., Zohuri 2017). The curatorial temperatures for terrestrial materials, including tissue samples and ice cores, include: $\leq-20{ }^{\circ} \mathrm{C}$ (the temperature of typical walk-in freezers in which physical processing and documentation takes place); $\leq-40^{\circ} \mathrm{C}$ for archival storage (e.g., of ice cores); and -80 to $-196{ }^{\circ} \mathrm{C}$ (liquid nitrogen) for biological samples (e.g., Anchordoquy and Molina 2007; Rissanen et al. 2010). Thus, with the exception of biological tissue storage, the field of Earth materials curation has not yet entered the realm of cryogenics.

\subsubsection{Past and Present Practices in Cold Curation of Astromaterials}

The expected range of temperatures required to preserve solar system materials spans from those needed for (water) ice cores to cryogenic. Cold curation and sample handling of astromaterials has been done to a limited extent at NASA-JSC over several decades. Several Apollo 17 samples were initially processed under $\mathrm{GN}_{2}$ in a processing cabinet at room temperature for about a month before being transported to cold storage $\left(-20{ }^{\circ} \mathrm{C}\right)$ where they have remained. Furthermore, the US Antarctic meteorite collection utilizes cold storage of new Antarctic meteorites, and initially used cold stages in a nitrogen glovebox for cold sample handling. According to Annexstad and Cassidy (1980) "The specimens are transferred from a small staging freezer to the processing cabinet. A specially constructed stage, cooled by liquid nitrogen, is used to keep the sample frozen while an initial cold chip is removed 
from the meteorite. This chip is immediately returned to freezer storage for future experiments when a frozen piece may be required. The parent meteorite is then allowed to warm to ambient temperature naturally in the cabinet's dry $\mathrm{GN}_{2}$ environment." In the first few years of Antarctic meteorite handling at JSC, the staff gained experience with storing and handling samples frozen, using a cold processing plate in a cabinet and using a cold storage room. Although some hardware was assembled to do this, it became clear after detailed tests that this was not an effective way to handle samples due to the difficulty of keeping samples cold while still allowing dexterity of the sample processor, length of time required to process individual samples, and overall expense. The cold processing approach was abandoned at JSC in 1979, after review and discussion by the Meteorite working Group (MWG) (Righter et al. 2014).

More recently, insights into the benefits of curation and processing under cold conditions have been gained from the collection, curation, and study of the Tagish Lake meteorite (Herd et al. 2016 and references therein). Tagish Lake is a unique carbonaceous chondrite that fell January 18, 2000 onto the frozen surface of the eponymous lake in northern British Columbia, Canada. The meteorite was collected about a week after the fall, and collection was done without direct hand contact; more significantly, the meteorite specimens were kept below $0{ }^{\circ} \mathrm{C}$ after collection and during subsequent transport to curation facilities (Herd et al. 2016). The cold ambient temperatures at the location of the fall, coupled with the care with which the collection and subsequent curation were carried out places Tagish Lake among the most pristine meteorites ever collected (Herd et al. 2016). The meteorite is a type 2 carbonaceous chondrite with affinities to CM and CI meteorites (Blinova et al. 2014; Zolensky et al. 2002), and contains among the highest concentrations of organic matter measured in meteorites (Alexander et al. 2014; Grady et al. 2002; Herd et al. 2011). The pristine nature of the meteorite, coupled with the curation methods used to preserve it, have yielded new insights into the formation of nanoscale organic globules in the coldest regions of the protoplanetary disk (e.g., Nakamura-Messenger et al. 2006) as well as the role of asteroid parent-body aqueous alteration in the modification and synthesis of organic molecules (Herd et al. 2011; Hilts et al. 2014).

The majority of the Tagish Lake meteorite specimens are stored at $-30{ }^{\circ} \mathrm{C}$ and processed within the Subzero Curation Facility for Astromaterials at the University of Alberta; this facility houses an Ar glovebox within a walk-in freezer maintained at temperatures of -10 to $-15^{\circ} \mathrm{C}$ (Herd et al. 2016). While there are no indications that the Tagish Lake meteorite contains water ice or other such volatiles, these conditions of storage and handling are justified by the discovery of especially volatile and/or reactive organic species (e.g., formic acid, naphthalene, and styrene; Hilts et al. 2014). Challenges and limitations of the Subzero Curation Facility include: mitigation of glovebox leaks, user comfort, and the extreme dryness of the Ar atmosphere, which would result in the sublimation of water or other ices from the samples (Herd et al. 2016). However, the facility achieves the goal of enabling documentation and processing of pristine astromaterials under low temperature in an inert atmosphere. The low-temperature curation of the Tagish Lake specimens reduces reaction rates, preserves intrinsic (volatile) organic compounds, and discourages microbial activity (Herd et al. 2016) — requirements that are desirable for returned samples from organic-rich asteroids, cometary nuclei, Mars, or other volatile-rich returned sample targets (lunar poles, icy moons, etc.) as discussed below. 


\subsubsection{Volatile-Rich Samples from the Lunar Poles}

The lunar poles are high-priority targets for sample return due to the possibility of significant quantities of water-ice and other volatiles in permanently shadowed regions (PSRs). Remote sensing data indicates that volatiles comprise up to several weight percent of materials in PSRs; the composition of the volatiles in the crater Cabeus' PSR included $\mathrm{H}_{2} \mathrm{O}, \mathrm{CO}_{2}, \mathrm{CO}$, $\mathrm{H}_{2} \mathrm{~S}, \mathrm{CH}_{4}, \mathrm{OH}, \mathrm{SO}_{2}, \mathrm{NH}_{3}, \mathrm{C}_{2} \mathrm{H}_{4}$, and $\mathrm{CH}_{3} \mathrm{OH}$ (Colaprete et al. 2010; Gladstone et al. 2010). This mix of compounds present a complex curatorial challenge, and even more so in the presence of local regolith/silicates (largely anorthosite or basalt). The volatiles detected by LCROSS have a range of condensation temperatures, and a subset are highly reactive in the presence of silicate minerals. If the solid and volatile components of a lunar PSR sample are stored together, therefore, a mixed-phase, highly reactive sample will likely result. The preservation of a lunar polar sample would therefore be maximized by separating the solid and volatile components and storing them in that configuration for the long-term.

The presence of numerous reactive species presents several additional challenges. First, the corrosive nature of $\mathrm{H}_{2} \mathrm{~S}$ limits the materials to which the sample can be exposed without alteration. Materials will therefore need to be selected that accommodate the curatorial requirements for isolation (materials should not significantly contract under cold temperatures), durability during sample processing/preliminary examination, and particulate contamination. Second, volatile-rich samples often contain gases that are hazardous to humans, even at low concentrations (e.g., $\mathrm{CO}, \mathrm{H}_{2} \mathrm{~S}, \mathrm{SO}_{2}, \mathrm{NH}_{3}$ ). This additional risk-on top of the existing particulate exposure risk from solid samples - may require the use of respirators or special masks during preliminary examination and curatorial operations. The need to minimize leakage from curatorial hardware (gas containers, analytical equipment, gloveboxes, etc.) will be significantly higher for volatile-rich samples; because they will operate at cold temperatures, proper materials selection from the component to the system level will be a top priority.

\subsubsection{Cometary Nucleus Samples}

The preservation of a cometary nucleus sample lies at the extreme end of cold-curation storage requirements because the sample would contain hypervolatiles including noble gases, nitrogen, and oxygen (Bieler et al. 2015; Le Roy et al. 2015), although the retention of these gases would only likely be achieved if they are trapped within solid ices of primarily $\mathrm{H}_{2} \mathrm{O}, \mathrm{CO}$, and $\mathrm{CO}_{2}$. Insights from the ROsetta Spectrometer for Ion and Neutral Analysis (ROSINA) instrument, which measured volatiles in the coma of comet 67P/ChuryumovGerasimenko demonstrate that, while dominated by water, the nucleus of 67P includes a wide range of volatile compounds, including molecular oxygen, $\mathrm{CO}, \mathrm{CO}_{2}, \mathrm{HCN}, \mathrm{H}_{2} \mathrm{~S}, \mathrm{CH}_{4}$, and many others (Bieler et al. 2015; Le Roy et al. 2015). Curation of these ices, which would almost certainly be intimately mixed with non-volatile, fine-grained silicate, oxide, sulfide and more refractory organic materials, would require significant technological development for cryogenic curation - assuming that the sample could be collected and returned to Earth under cryogenic conditions in the first place. Various options for the return of cometary nucleus volatiles have been studied, including cryogenic sample return, for which significant technical challenges exist (Veverka 2010b). Allowing volatile components to be released by warming a comet nucleus sample and capturing them in a separate container removes the need for cryogenic handling (Veverka 2010a), which was the approach proposed for the CAESAR mission concept to comet 67P. No truly cryogenic sample return missions are planned at the time of this writing. 
Fig. 1 The overlapping challenges of cold curation and biological containment present unique challenges for future astromaterials curation efforts

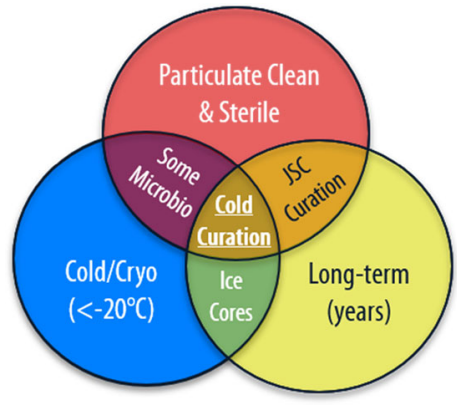

\subsubsection{Biological Samples}

Curation facilities may be required to curate biological samples as part of a contamination knowledge collection from the spacecraft build and sampling of flight hardware. This requirement is currently in place for the Mars2020 mission, which may be the first leg in a Mars Sample Return Campaign. Although there are no requirements that the martian samples be kept cold, biological sampling during the spacecraft build and of the flight hardware includes microbiological samples, including swab samples, liquids, isolated pure cultures of bacteria and fungi, and DNA samples. The requirements for long term preservation of these biological samples varies with sample type and intended use. We will discuss two broad sample types: (1) Samples preserved for later growth and (2) samples intended for molecular analysis like DNA sequencing.

The guidelines for preserving bacteria and fungi for later cultivation are well established (CABRI 1998). Bacteria should be placed in a protective solution of $15-50 \%$ glycerol by volume and frozen at $-80{ }^{\circ} \mathrm{C}$. Commercially available products like cryobeads should be used to improve long-term viability. If viability needs to be maintained for $>5$ years, samples should be frozen at $-130{ }^{\circ} \mathrm{C}$. Some species of bacteria and fungi can be freeze dried with liquid nitrogen and stored at $4-8{ }^{\circ} \mathrm{C}$. It is important to test the survivability of each strain prior to committing to a preservation method.

Preservation of swabs, liquids, witness plates, or extracted DNA for later analysis is less straightforward. As a general rule, colder is better, but there is little consensus on what temperature is best. There is some evidence that storing samples at too low a temperature can cause more damage than it prevents (Anchordoquy and Molina 2007; Vaught and Henderson 2011). Rapid changes in DNA sequencing technology make it very difficult to predict how samples will be handled in the future (Reuter et al. 2015). DNA extraction techniques are also evolving and can have significant effects on sample quality (Dauphin et al. 2009; Mitchell and Takacs-Vesbach 2008; Rose et al. 2011; Zielińska et al. 2017). Barring additional research, the best strategy is to store unprocessed samples alongside extracted DNA so that future researchers have options for what to analyze.

Future sample return missions from icy moons will incorporate both the biological sensitivity of a Mars Sample Return (MSR) campaign and the temperature sensitivity of lunar or cometary samples (Fig. 1). Therefore, even if MSR does not have a low-temperature storage requirement, it is inevitable that biological containment and cold curation will eventually be needed concurrently. The challenges of operating a bio-safety level 4 (BSL-4) facility at cold temperatures are unique to astromaterials curation, and will need to be addressed in the coming years. Many materials suitable for biological containment (e.g., many plastics) become brittle at temperatures at or below the freezing point of water. The additional presence 

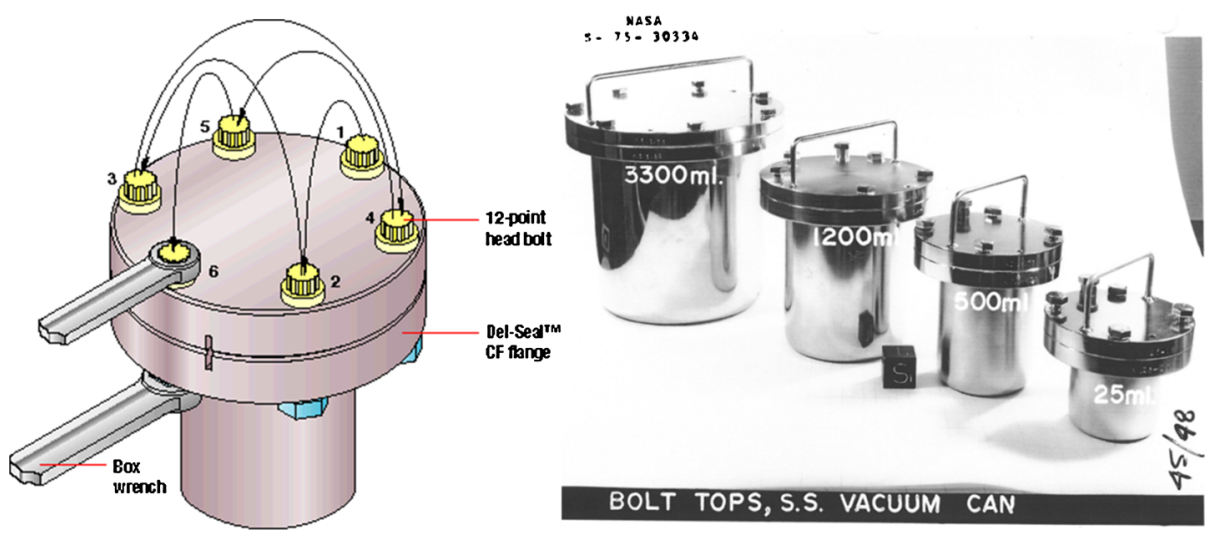

Fig. 2 Sketch of typical assembled conflate flange (left). This design is very similar to the specialty "bolt top containers" used for the Apollo program sample storage in 1969 to present (right), except that these are now commercially available, and in various sizes and materials, as explained above

of salts (chlorides, sulfates, etc.) may pose challenges to the selection/durability of metal components. The overlapping requirements for sterility, particulate cleanliness, temperature control, leak prevention/sample isolation, gas safety, and curator comfort will need to be met in the coming years as exploration efforts at Europa, Enceladus, and other icy moons intensify.

\subsection{Curation of Organics and Organic-Rich Materials at Room Temperature}

Experience with the curation of the organic-rich Tagish Lake meteorite (Sect. 3.1), has provided ample evidence for the value of cold curation in the preservation of organics and organic-rich materials; namely, the retention of volatile organics, the mitigation of volatile organic contaminants, and the suppression of metabolism by any microorganisms in the curation facility (Herd et al. 2016). However, cold curation is not a requirement for the storage of organic-rich materials. For example, curation planning for OSIRIS-REx turned instead towards hermetically sealed storage of samples to preserve organics (Dworkin et al. 2018). This approach had some precedent with the Apollo missions samples (Fig. 2), avoids the potential contamination and time and dexterity intense processing issues associated with cold curation, and is cost effective utilizing known commercially available and tested hardware and approaches.

When handling organic-rich or organic sensitive materials the use of plastics should be extremely limited. PTFE or Teflon is acceptable in some situations, but glass or metal is preferable. Prior to use, tools and sample containers should be combusted at $500{ }^{\circ} \mathrm{C}$ to remove organic contaminants. Long-term storage of organically sensitive samples should use well-characterized glass baked at $\geq 500{ }^{\circ} \mathrm{C}$ wherever possible (e.g., Grosjean and Logan 2007; Peters et al. 2005; Sherman et al. 2007). Furthermore, frequent microbial monitoring of labs where organic-rich samples are stored and processed is critically important. Moreover, metagenomics studies of any microbes recovered from curation labs that house and process organic-rich samples will be important, particularly for microbes that can metabolize under anaerobic conditions. The primary goal of these metagenomic studies would be to characterize the metabolic function of these anaerobic microbes to understand how they might alter the samples if they are inadvertently introduced to the samples. This is particularly important for organic-rich sample collections stored at room temperature. 


\subsection{Future Restricted Earth Return Missions}

In the 50 years since the Apollo 11 launch, advancements in knowledge and technology allow for not only unprecedented scientific investigations of extraterrestrial samples but also a greater understanding of the potential hazards of sample exposure or release into the environment (e.g. extraterrestrial life). However, in the case of a biological health hazard, more precautions are required, not only to protect the samples from Earth, but also to protect Earth from the samples. Under the UN Space Treaty of 1967, the Committee on Space Research (COSPAR) maintains a planetary protection policy at the international level for all space faring nations. The policy provides "international standard on procedures to avoid organic-constituent and biological contamination in space exploration" (COSPAR Planetary Protection Policy March 2017). The policy also promotes the prevention of "adverse changes in the environment of the Earth resulting from the introduction of extraterrestrial matter" as stated in the UN Space Treaty.

For the United States, the NASA Planetary Protection Office in the Office of Safety and Mission Assurance provides the policies and requirements for all NASA exploration missions regarding forward and backward control of biological contamination. NASA Policy Directive (NPD) 8020.7G, Biological Contamination Control for Outbound and Inbound Planetary Spacecraft, complies with the UN Space Treaty and COSPAR planetary protection policy stating "the Earth must be protected from the potential hazard posed by extraterrestrial matter carried by a spacecraft returning from another planet or other extraterrestrial sources”. NASA Procedural Requirements (NPR) 8020.12D, Planetary Protection Provisions for Robotic Extraterrestrial Missions, outlines requirements for meeting the NPD 8020.7G as well as specifies planning documents and reviews for Category V Restricted Earth Returns. The Planetary Protection Office classifies any "samples from solar system bodies that may harbor indigenous life" as Category V: Restricted Earth Return. Although there are currently three bodies with this designation (Mars, Europa, and Enceladus), this number can change in either direction as more information about any particular planetary body is gained. For example, during the first three Apollo sample return missions (Apollo 11, 12, 13, and 14), the Moon was considered Restricted. Consequently, the Apollo 11, 12 , and 14 samples and astronauts were quarantined upon arrival while health assessments and biohazard tests were performed. However by Apollo 15, which launched just over two year after Apollo 11, the Moon was reclassified as Unrestricted and the final three Apollo missions (Apollo 15, 16, and 17) proceeded without the same level of biohazard Planetary Protection precautions.

The scientific community has identified Mars Sample Return (MSR) as a high priority sample return activity for many years, and support for such an endeavor has waxed and waned over the last few decades. Current efforts relating to MSR are focused on a multimission campaign, the first of which is the Mars 2020 rover mission to Jezero Crater. At the time of writing, no space agency has fully committed to returning the samples that will be collected by Mars 2020, but NASA and ESA are discussing the possibility of forming a partnership to complete the campaign and decisions are anticipated to be made in the year 2020 . Due to both Planetary Protection and Science requirements, the Mars 2020 rover mission has the most stringent inorganic, organic, and biological contamination control requirements of any sample return mission in history. Strategies for satisfying these and other requirements related to MSR and Restricted Earth Return in general are described below. 


\subsubsection{Facility Preparation}

Infrastructure Samples returned from any planetary body designated as Restricted must be contained within a Biosafety Level 4 (BSL-4) facility until it can be demonstrated that either (1) the samples do not pose a threat to life on Earth or (2) the samples have been adequately sterilized for release (Rummel et al. 2002, NASA technical publication 211842). The requirements and processes associated with biohazard testing and/or sterilization are developed specifically for each mission and each set of samples. International space treaties with the United States, COSPAR planetary protection policies, and NASA planetary protection policy directives and requirements do not impose any specific design requirements on a biocontainment architecture or BSL-4 facility. The policies simply state that the Earth must be protected from the potential hazard posed by extraterrestrial matter and microbial containment is required on Category V (sample return) Restricted Earth Returns. The U.S. Dept. of Health and Human Services traditionally has jurisdiction of design and operating requirements for a BSL-4 facility in the United States. The "Biosafety in Microbiological and Biomedical Laboratories", 5th Edition (Dec. 2009) authored by the U.S. Department of Health and Human Services: Public Health Service, Centers for Disease Control and Prevention, and the National Institutes of Health; HHS Publication No. (CDC) 21-1112 (hereafter BMBL, 2009), houses the primary recommendations, standards, and design requirements for all BSL labs. Under this regulation, any related agents with unknown risk of transmission are classified to be under BSL-4 containment. Presumably, an extraterrestrial or unknown pathogen would require, at minimum, a BSL-4 containment. At this time, we cannot predict what other federal or international agencies may wish to impose additional guidelines and requirements and/or request jurisdiction of a NASA BSL-4 sample return lab. For example, the National Institute of Health (NIH) imposed additional design requirements at the Galveston National Lab beyond the BMBL requirements. The World Health Organization also has guidelines and requirements for BSL-4 laboratories and the Dept. of Agriculture has claimed some jurisdiction of extraterrestrial soils. For the Apollo Program in January 1966, the Interagency Committee on Back Contamination (ICBC) was established to include the CDC with Dr. David Sencer of the CDC as chairman, Department of Agriculture, Department of the Interior, Department of Health, Education, and Welfare, National Academy of Sciences, and NASA, which imposed strict requirements on the construction of the Lunar Receiving Laboratory (LRL), JSC Bldg. 37. Therefore, historically, other agencies have been involved in the construction and operations of such a BSL-4 type lab.

One of the major challenges in designing a facility for Restricted Earth Return Missions is the integration of the Contamination Control (CC) requirements necessary to protect the samples from terrestrial contamination and Planetary Protection (PP) requirements necessary to protect the Earth and its inhabitants (all life: from humans to animals to plants, etc.) from a possible extraterrestrial pathogen (e.g., microbes, viruses, or prions). While walls can act as physical barriers for protections, developing the proper pressure differentials inside and outside the laboratory is vital (Fig. 3). Unlike the curation of traditional unrestricted samples, which utilizes positive pressure gradients to protect the samples from contamination, BSL-4 facilities rely on negative pressure gradients to protect the scientists and general public from the samples. Although there have been a number of possible iterations demonstrated in the Draft Test Protocol (Rummel et al. 2002; Fig. 3), the presumed baseline requirement is that the samples must be contained within a BSL-4 Facility (BMBL 2009). In order to best protect the samples and Earth, redundancies are built into the design schematic (Fig. 3). Specifically, not only will the entire cleanroom laboratory be constructed within a BSL-4 facility, but the use of a Biosafety Cabinet (BSC) III double walled isolator for 


\section{Standard \\ Astromaterials Curation \\ Sample Containment Positive Pressure Cascade}
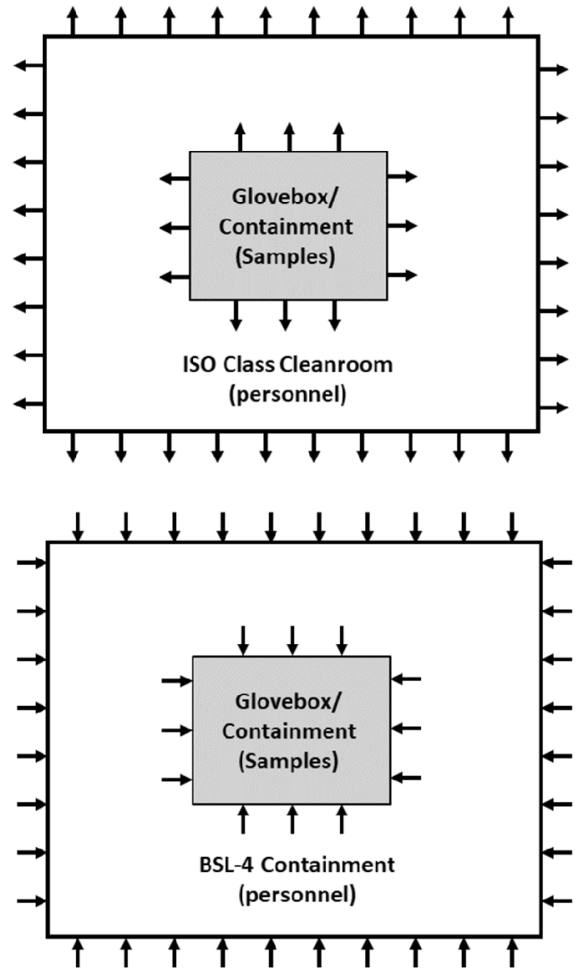

Fig. 3 Comparison of differential pressure gradients used for containment of astromaterials samples from bodies designated as restricted Earth return (adapted from Rummel et al. 2002: 6, Fig. 1): (1) The top schematic represents a standard sample containment design for unrestricted sample return missions. In this configuration, the highest pressure is situated inside a positive pressure glovebox or containment isolation chamber and pushes out to a lower pressurized cleanroom. The cleanroom is also under positive pressure relative to the outside of the lab. This positive pressure cascade is designed to mitigate the infiltration of outside or laboratory contamination to the astromaterials samples. (2) The middle schematic is a standard BSL-4 containment design for working with hazardous biological pathogens. In this particular configuration, the glovebox or containment isolation chamber that houses the biohazard is under negative pressure relative to the laboratory space. In addition, the laboratory is also under negative pressure relative to outside of the lab. This negative pressure cascade is engineered to protect the outside environment from a release of any biohazard. (3) The bottom schematic is the current design concept for a restricted Earth return sample containment that combines both of these technologies. The containment isolation chamber that houses the samples is designed with a double wall and the interstitial space is filled with a high purity gas at a higher pressure relative to the contained isolated samples and the cleanroom. The pressure between the cleanroom and containment is still under a negative pressure differential to maintain BSL-4 containment standards, but any leakage would be the high purity gas that pushes into the containment and out to the laboratory cleanroom. In addition, the cleanroom laboratory would be a positive pressure cascade with a negative differential pressure plenum barrier to maintain BSL-4 containment to the outside environment of the facility

sample processing within the cleanroom will add an additional level of protection with the corresponding differential pressure scheme. For current BSL facilities in the U.S., a Class III BSC glovebox gastight (leak rate) criterion is $<1 \times 10-5 \mathrm{cc} / \mathrm{s}$ with $100 \%$ He tracer gas under $3 \mathrm{inH}_{2} \mathrm{O}$ pressure in the cabinet (Stuart et al. 2012). Dependent on mission science requirements, specialized double walled glovebox or containment seals could be required for maintaining nitrogen or other inert gas environment purity under negative pressure. Nonglove storage isolators can achieve a He leak rate of $<1 \times 10-7 \mathrm{cc} / \mathrm{s}$. However, achieving 


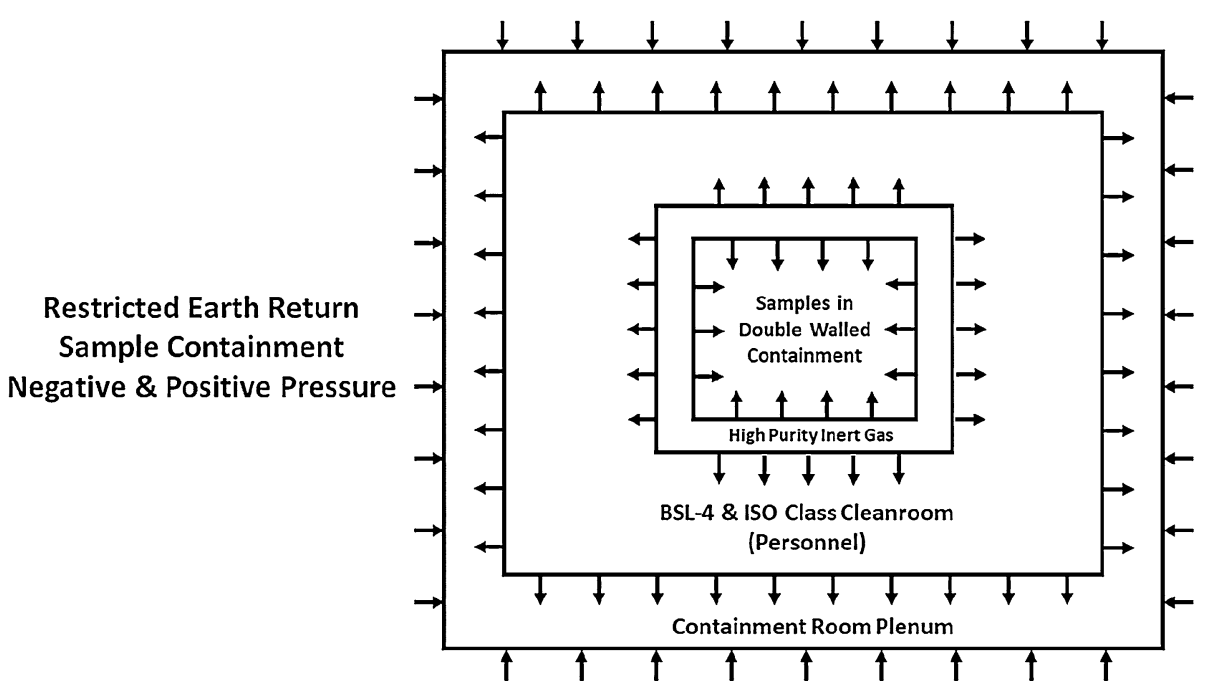

Fig. 3 (Continued)

a better leak rate on double-walled isolated containment may require additional engineering development and challenges. While there have been some studies exploring how these requirements could be implemented (Beaty et al. 2009), these studies need to be updated to reflect some significant shifts in possible facility usage (e.g. no animal studies, long-term use, multi-mission use).

Functional Laboratory Design The verification of extinct or extant life within a sample may require the examination of organic compounds within the samples. As such, not only is it vital to ensure no terrestrial biological contamination occurs during sample handling and storage, but the amount of organic contamination must also be minimized. One of the main ways to do this is through the selection of manufacturing equipment and laboratory space with proper materials that have little to no potential to outgas or shed particles. This will require the use of mainly 300 series stainless steel and Teflon in areas with intimate contact with the samples. For this reason (as well as offering additional differential pressure gradients), double walled isolator cabinets are the likely choice for the handling of these materials since the suits utilized in BSL-4 facilities and the glovebox gloves could contaminate the samples with organic matter and make life detection more difficult (Vrublevskis et al. 2018a; Holt et al. 2019). Although the utilization of a double-walled isolator (DWI) helps to mitigate the contamination risk, it requires a significant advancement in remote or robotically assisted manipulation since manual manipulation via a glove port could compromise the organic CC requirements due to material outgassing. Although there is work being done in Advanced Curation related to small particle handling, there are other groups investigating hepatic feedback remote handling, specifically for Mars Sample Return (Vrublevskis et al. 2018 b). In addition to robotic sample manipulators, and any analytical equipment should be developed to allow scientists to manipulate samples and run analyses remotely.

A further complication of returning samples from a restricted planetary body is the unknown long-term space requirements of the collection. Although Apollo samples were eventually deemed safe and released (two years after the first samples were returned), this will not necessarily be the case for future restricted Earth return missions. If a potential or actual health hazard is found, or if there are too many concerns about unknown unknowns, 
samples may never be released from containment. Therefore, multiple facilities and sample use strategies would need to be developed to conduct science in containment. One way to approach this is to construct a modular BSL-4 facility that has walls that can be shifted to accommodate the addition of new analytical instrument suites and other long-term curation/scientific needs.

Integration of Cleaning and Sterilization Techniques The safety of the samples and the technicians and environment at large will require not only a well-designed facility, but also the integration of cleanliness and sterilization protocols. While there are standard sterilization techniques (e.g. heat, peroxide, formaldehyde) and cleaning procedures (e.g. IPA, UPW), there is not one standard procedure that can do both simultaneously. In the case of MSR, where a major part of "life-detection" will rely on DNA extraction and not viability, any unviable biological matter remaining will compromise the samples and studies. Due to these considerations, strategies for integrating these procedures are underway. A similar strategy as that taken for flight hardware will be employed, clean the materials first and then sterilize without generating contamination. Although the Mars 2020 Mission has integrated the use of vapor phase hydrogen peroxide (VHP), they are using heat sterilization on the sample intimate hardware. However, given the specs of isolator cabinets (e.g. size and mechanics) and the systematic sterilizations needed to avoid cross-contamination and ensure safety, heat sterilization is not a viable option.

The need to integrate the isolator cabinets into the facility's infrastructure will mean that they will have to be cleaned and sterilized in place. For this we can draw upon best practices used in Curation Glovebox Laboratories and BSL-4 Cabinet Laboratories. For initial final cleaning/sterilization, a standard UPW/IPA cleaning procedure would likely be utilized (see Sect. 3.7) followed by sterilization utilizing an ultra-pure hydrogen peroxide solution. Given the harshness of the high concentration peroxide required (35\%), amount of residual moisture after sterilization is complete, and its limitations in sterilizing instrumentation due to unexposed surfaces, a new technique is being considered. Not only does ionized hydrogen peroxide only require a solution of $8 \%$ hydrogen peroxide, it more easily permeates instrumentation, and does not leave a liquid residue (Webb 2011; Grimaldo 2017). Although more research is required to confirm sufficient sterilization without generating long-term corrosion or systemic contamination, the outlook is promising.

\subsubsection{Collection of Baselines for Science and Planetary Protection}

The concurrent requirements for sterility, particulate and organic volatile cleanliness, temperature control, leak prevention/sample isolation, and gas safety will need to be met in the coming years as exploration efforts at Mars, Europa, and Enceladus come to fruition. The classification of a mission as Category V Restricted Earth Return not only adds more CC and PP constraints (https://planetaryprotection.nasa.gov/categories), it also broadens the scope of required CK (Fig. 4) to include biological witness materials. Not only does this require more rigorous sets of samples, unlike other collections, which only require storage in an inert ultra-pure gaseous environments (e.g. nitrogen), biological CK will also require samples to be frozen (see Sect. 3.1).

While all scientific investigations of returned samples are highly sensitive to terrestrial contamination, contamination is especially detrimental where studies of extant or extinct extraterrestrial life are concerned. The proper collection, storage, and cataloging of Contamination Knowledge (CK) associated with the production and assembly of the spacecraft, rover, lander, orbiter, and/or sampling system will be vital to these investigations. Without 


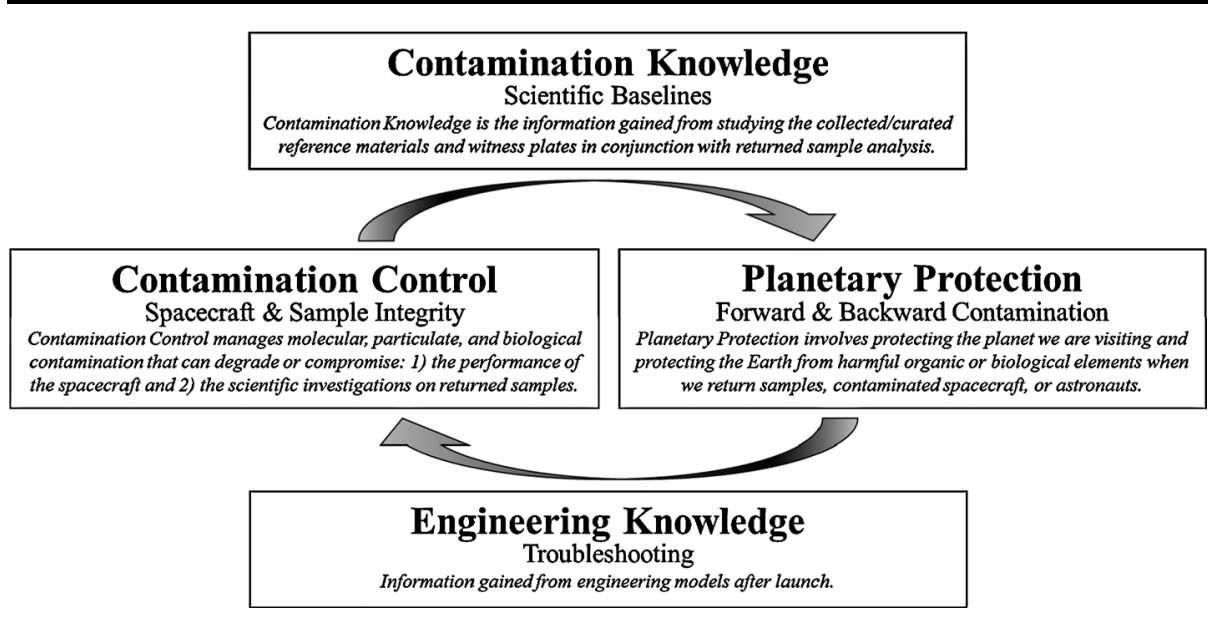

Fig. 4 Cartoon illustrating the categories of samples needed for testing and verification of spaceflight missions. Each type of sample serves a different purpose and hence requirements for each sample collection related to these categories must be defined

a well-constructed and curated CK collection, the baseline for contamination within the returned samples cannot be established. Therefore, after mission inception and design, the development of the CK collection as part of a mission's curation plan (CP) should occur in conjunction with the mission's CC and PP plans. The CP, CC, and PP plans and implementation of these plans during ATLO and Earth receiving operations are paramount to the ultimate value of the returned samples.

Technological advancements to instrumentation are continually progressing with greater precision and accuracy for sample measurements, especially in the field of microbiology. An array of CK samples must be made available to scientists once restricted samples are returned to Earth, and those samples should be preserved in such a manner that they can be analyzed by instrumentation that was not invented at the time of their initial storage. Collecting and curating unanalyzed/unaltered samples will minimize the possibility that current analysis and extraction techniques destroy or alter the samples or otherwise inhibit yet to be developed measurements. Some of the types of biological CK samples the NASA Curation Office requires for restricted Earth return missions include:

(1) Un-analyzed/Un-altered dry swabs and wipes in sterile containers stored at $\leq-80^{\circ} \mathrm{C}$.

(2) All recirculation filters from the clean rooms used for spacecraft and spacecraft hardware assembly and all filters from the laminar flow benches used to assemble sample intimate hardware, packaged in sterile Teflon bags and frozen at $-80{ }^{\circ} \mathrm{C}$.

(3) Witness plates collecting airborne contamination within the assembly cleanrooms stored at $\leq-80{ }^{\circ} \mathrm{C}$.

\subsection{Sample Processing Cabinets Under Vacuum}

Historically, vacuum processing of samples was employed for primary processing of Apollo lunar materials. First envisioned in 1964, the 3 story "High Vacuum Complex" integrated into JSC bldg. 37 Lunar Receiving Laboratory between 1967-1968 was a series of connected glovebox isolation chambers operating at $10^{-6}$ torr vacuum environment to decontaminate, process, and store samples (Calaway et al. 2014; White 1976). Although vacuum processing takes a very direct approach to minimizing sample contamination by avoiding sample 


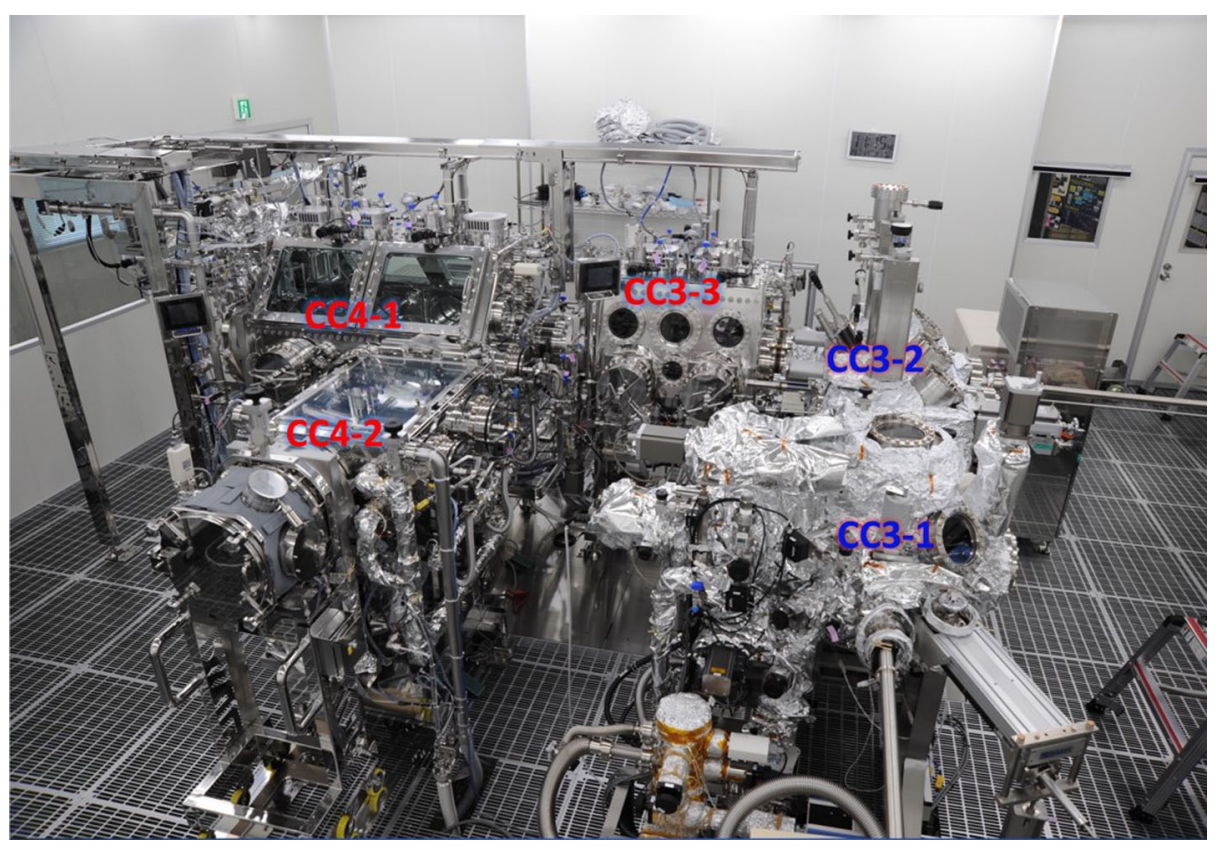

Fig. 5 A bird's-view image of the sample-handling system for Ryugu samples. The system consists of five chambers-CC3-1, CC3-2 CC3-3, CC4-1 and CC4-2

contact with gases, the process is inherently difficult. Maintaining vacuum in large processing cabinets requires constant pumping and the use of cold traps to remove unwanted pumping oils and other contaminants, which renders gloves stiff with attendant processor fatigue, and any leaks in the system tend to introduce relatively humid ambient air. There is also the danger of rapid pressure loss through mechanical failure, which would introduce significant contamination and poses a physical risk to processors. Unfortunately, the F-201 processing glovebox chamber was prone to leaks and glove failures as well as difficulties in using vacuum hardware with an increasingly large volume of lunar samples, which ultimately drove the high vacuum complex to be used only for Apollo 11 and 12. For Apollo 14 onward, the high vacuum complex was replaced by a series of positive pressure gaseous nitrogen gloveboxes called the Sterile Nitrogen Atmospheric Processing (SNAP) Line and Nonsterile Nitrogen Processing Line (NNPL) (Reynolds et al. 1973; Simoneit et al. 1973).

The JAXA Extraterrestrial Sample Curation Center (ESCuC) in Sagamihara, Japan has successfully employed vacuum storage for samples returned from the Hayabusa mission to asteroid Itokawa and is planned for JAXA's Hayabusa2 mission to carbonaceous asteroid Ryugu that is currently in flight (Okazaki et al. 2017; Sawada et al. 2017; Yada et al. 2014). In the case of the Hayabusa samples, all the sample handling processes occurred in purified gaseous nitrogen following the sample container opening process under vacuum conditions (Yada et al. 2014). Installation of a newly-developed sample-handling vacuum processing clean chamber (CC) was completed in October, 2018 at ESCuC (Okazaki et al. 2017; Sawada et al. 2017; Fig. 5) two years prior to sample return. The entire sample-handling system in ESCuC consists of five chambers-CC3-1, CC3-2, CC3-3, CC4-1 and CC4-2. The returned sample container will be first connected to $\mathrm{CC} 3-1$ for opening in a vacuum, and will be transferred to CC3-2 for vacuum-handling of samples. The container will be 
then transferred into CC3-3, where the sample handling environment will be changed from vacuum to purified gaseous nitrogen. Further handling of samples will be done in purified nitrogen in CC4-1 and CC4-2.

\subsection{Sample Processing Cabinets with Remote Participation of Scientists}

It is rather common for scientific investigations with astromaterials to require a specific sample or specific portion of a larger sample for subsampling. Principle investigators will occasionally travel to a curation facility to pick-out samples, provide input on sample subdivision, and/or sample preparation. This requires that the PI arrange a visit to the facility to communicate directly with the curatorial processors on which samples to pull and ultimately choose for study. In some cases, travel can take a significant amount of time and cost. Today, communication technology can almost eliminate the need for the PI to travel to the facility with the integration of "Live" real-time video conferencing sessions with remote scientists.

NASA JSC has experimented with this technology during the Stardust preliminary examination in 2006 and again in 2014 with the retrofit of one of the Apollo 16 Lunar processing cabinets (Calaway 2015). In this most recent technology demonstration, a Leica DMS1000 Macroscope and Axis Pan-Tilt-Zoom (18× optical zoom) IP camera system was integrated into the Lunar processing glovebox. The Axis camera and macroscope were mounted on the outside of the glovebox and focused through glass. This was done to eliminate the concern for cross-contamination from the two systems. The Axis camera was mounted to the top of the glovebox looking through the lighting window and could be used for situational awareness of processing or zoomed in to look at sample splits. The Leica Macroscope was mounted above the PI observation window at the end of the glovebox. A sample was placed onto a jack-stand and the macrosope could focus on the sample through the glass window.

While both of these commercial-off-the-shelf (COTS) products offer video streaming capabilities, the video integration was complicated by the JSC firewall and mandated government IT security requirements. In addition, remote wireless connections were hampered by the thick walls of the curation facility. For both the systems, the video needed to be securely accessed outside of JSC. Therefore, the video stream was required to push through the JSC internal firewall to the JSC public zone and then pass through another firewall to get to the internet for public access. The Axis camera browser software is capable of secure viewing with passwords, and the IP address would be routed accordingly by our internal IT group. For the macroscope video stream, a streaming service like YouTube or USTREAM from the DMZ could be used. For the tech demo, a dedicated USTREAM account was set-up. At the March 2015 Lunar and Planetary Science Conference, we demonstrated this system. We successfully live-streamed the Leica Macroscope and Axis camera real-time images from the NASA JSC Lunar laboratory to the Marriot Hotel in The Woodlands, Texas. However, for this test we used the VPN network access to simplify the test due to time constraints. Lunar curation now has all the equipment and tools needed to set up a permanent video conferencing with external PIs during a video or teleconference. In the future, more collections could integrate this type of COTS technology to reduce time and travel costs where appropriate.

\subsection{Small Particle Handling}

One particular objective of advanced curation efforts is the development of new methods for the collection, storage, handling and characterization of small particles. In this context, "small" refers to microscale particles, typically between one and several hundred microns 
in diameter (though submicron interstellar particles will be analysis targets in the future). Particles in this size range include dust derived from comets and asteroids that is continuously accreted by the Earth, as well as material collected by robotic sampling missions and by astronauts during Apollo missions. The curation of small particles includes the unambiguous identification of particles in/on collection substrates, the transfer of particles between collection, analysis, and storage substrates, sample characterization, sample preparation/subdivision, and the preservation and documentation of samples in a publicly available catalog. Astromaterials Curation facilities in the United States, Russia, and Japan currently maintain several small particle collections (Allen et al. 2011): Lunar regolith fine-grained samples returned by Apollo astronauts and by Soviet Luna robotic spacecraft, Cosmic Dust that has been collected in Earth's stratosphere by ER2 and WB-57 aircraft, Comet 81P/Wild 2 dust returned by NASA's Stardust spacecraft, interstellar dust returned by Stardust, and asteroid Itokawa particles that were returned by the Hayabusa spacecraft. NASA and JAXA Curation offices are currently preparing for the anticipated return of two new astromaterials collections-asteroid Ryugu regolith collected by Hayabusa2 spacecraft in 2019 and returned to Earth in 2020, and asteroid Bennu regolith to be collected by the OSIRIS-REx spacecraft and returned in 2023 (Lauretta et al. 2017). A substantial portion of these anticipated returned samples are expected to consist of small particle components, and mission requirements necessitate the development of new processing tools and methods in order to maximize the scientific yield from these valuable acquisitions.

There are several aspects of microscale astromaterials curation that present challenges that are distinct from macroscopic sample curation. At scales of less than $100 \mu \mathrm{m}$, electrostatic and intermolecular forces dominate the behavior of particles. Particles adhere weakly to glass or tungsten needles via Van der Waals intermolecular forces, usually enabling transfer between analysis and storage substrates. These transfer operations are hindered by transient electrostatic forces. Triboelectric charging due to contact, separation, and frictional electrification (Matsusaka et al. 2010) is the primary mechanism by which particles are lost during transfer operations (although environmental and instrumental vibrations also contribute to sample loss during transfers), and these triboelectric effects become more severe in low-humidity environments. Hayabusa2 and OSIRIS-REx collections will be curated in sample processing cabinets purged with dry $\mathrm{GN}_{2}$, and developing methods for suppressing triboelectric charge accumulation in these dry environments will be critical for successful sample processing.

Sample characterization at the microscale also presents unique challenges. Typically, optical images of submicron to micron-sized particles do not provide sufficient information to investigators to make informed sample selections. Microscale particles are often imaged and characterized in scanning electron microscopes equipped with an energy-dispersive spectroscopic (EDS) detector for elemental characterization; such analyses are useful for investigators requesting samples with desired mineralogy and are necessary to distinguish true extraterrestrial material from terrestrial contamination for samples that are collected in the stratosphere. However, SEM analysis of microscale particles introduces an additional risk of loss due to sample charging from electron beam bombardment; additionally, some fragile organic and mineral phases may be potentially modified by e-beam characterization. In some instances (especially with rare samples), it may be necessary to subdivide a particle via ultramicrotomy or by focused ion beam (FIB) sample preparation. These methods must be carefully considered in order to avoid compromising the scientific integrity of the sample. The objective of advanced microscale astromaterials curation research is to better understand these challenges and to investigate tools, equipment, and methods that facilitate microscale sample processing. 


\subsubsection{Description of Tools and Equipment Used for Small Particle Handling}

Tweezers Commercially available, low-Ni stainless-steel tweezers (e.g. Dumont No. 5 Dumoxel) can be utilized to reliably manipulate samples as small as several hundred microns by hand. Smaller particles $(\geq 50 \mu \mathrm{m})$ may be manipulated by tweezers that are fixed to devices that enable mechanical or electrical actuation, especially when mounted on a micromanipulator. The NASA Curation Office at JSC has acquired two such devices manufactured by Micro Support Co., Ltd.; these devices are being used to investigate methods of particle removal from OSIRIS-REx contact pads. Challenges remain with small particle manipulation via tweezers (either by hand or by electrical/mechanical actuatable devices) due to lack of force feedback, and risk of deforming or fracturing particles with low tensile strength remains significant.

The use of micro-electro-mechanical systems (MEMS) microtweezers for particle manipulation (Keller and Howe 1997) have also been investigated. Initial experiments with these devices revealed similar force feedback limitations to stainless steel tweezers. In addition, silicon devices were more brittle, making removal and placement of particles on rigid substrates precarious operations that often resulted in the shattering of the microtweezers and the loss of the sample. Finally, the low-cost benefits due to mass production on a single wafer have so far not been realized, and the cost of these microtweezers has remained significantly more expensive than their stainless-steel counterparts.

Needles Manipulation of particles by fine-tipped needles is a technique that has been utilized by curators since NASA initiated the cosmic dust collection in 1981. Particles smaller than $20 \mu \mathrm{m}$ are typically transferred from a collection medium to an analytical substrate (e.g. beryllium disk or epoxy bullet) or to a storage container (e.g. concavity slide) using a microneedle made from glass or tungsten. With skill and practice, curation personnel can transfer particles as small as $5 \mu \mathrm{m}$ between substrates by hand using a glass or tungsten needle attached to a pin vise. However, involuntary hand motions on the order of $100 \mu \mathrm{m}$ make routine and reliable transfer tedious and precarious operations. For critical transfers, needles are attached to mechanical or motorized micromanipulators to improve transfer reliability and precision, while minimizing user fatigue. The intermolecular forces between the needles and the particles in this size range are typically sufficient to overcome repulsion due to triboelectric charge accumulation. Larger $(\geq 20 \mu \mathrm{m})$ particles have been more challenging to manipulate. When using the same glass and tungsten microneedles for particles larger than $20 \mu \mathrm{m}$, triboelectric charging effects significantly hinder the reliable manipulation of particles. We have recently observed that, by utilizing tungsten carbide needles with low taper ratios ( 3:1), particles as large as $200 \mu \mathrm{m}$ can be manipulated successfully. We speculate that these needles present greater contact surface area for intermolecular forces to capture particles, and that the needle shape may aid in the rapid redistribution of accumulated triboelectric charge; however, more tests are needed.

Glass and quartz needles are fabricated using micropipette pullers that concentrate heat at the center of a solid core glass rod or capillary tube while applying force to each end; this action creates two needles with submicron tips. When a capillary tube is pulled in such a manner, and the tip is carefully broken off, a micropipette is created. A vacuum can be applied to this tube, creating a microscopic version of vacuum tweezers. We have investigated utilizing such a system to manipulate particles that are too large to be handled by Van der Waals adhesion. Our initial results are that, while the vacuum tweezer system is very efficient at securing larger microscale particles, releasing the particle by removing the vacuum frequently results in sample loss. We speculate that the vacuum action induces strong triboelectric charging effects. 
Microscopes Stereomicroscopes possess imaging characteristics such as long working distance and laterally correct viewing that make them extremely well-suited for freehand and mechanically assisted manual manipulation of microscale particles. Stereomicroscopes from manufacturers such as Nikon, Leica, and Olympus utilize two main optical designsthe Greenough design, which has two optically independent light paths, and the common main objective (CMO) design, in which optically parallel light paths share a common objective (Zimmer 1998). Greenough designs are preferable in environments in which size and weight must be minimized, and where high magnification is not necessary or desired (e.g., suspended over a collector during cosmic dust harvesting). CMO optical designs afford increased magnification compared with Greenough type microscopes, and are utilized in more critical sample operations such as mounting particles onto analysis substrates.

For the manipulation of very small $(<10 \mu \mathrm{m})$ samples, upright microscopes equipped with geared XY stages are utilized. These microscopes are equipped with long-working distance objectives capable of providing up to 500X magnification. The geared, manual XY stage is coupled to the Z-focus mechanism that raises and lowers beneath a stationary objective; this enables movement in $\mathrm{X}, \mathrm{Y}$, and $\mathrm{Z}$ independent of objective position. By placing a needle at the focal point of the objective, it is possible to transfer microparticles between substrates by moving the stage rather than by moving the needle.

Digital microscopes are particularly useful in processing environments where stereo or upright microscopes would be inconvenient-for instance, in a $\mathrm{N}_{2}$ sample cabinet or a temperature-regulated environment. They also introduce the potential to perform curation activities remotely, reducing contamination risks and operator fatigue. JAXA's Hayabusa sample processing cabinet uses three digital microscopes - two mounted inside the cabinet, and one mounted externally - to image particles during transfer operations. Digital microscopes are best utilized with micromanipulator assisted particle transfers, especially if the microscope suffers from image lag.

Micromanipulators Micromanipulators are mechanical, hydraulic, and motorized/ electrical devices that enable the precise handling of microscale samples. Most commercially available micromanipulators have three axes of motion, with motorized versions often providing a virtual fourth axis of motion (which is desirable for performing microfluid injections). Mechanical micromanipulators often use a combination fine-pitched screw mechanisms and linear guide rails to achieve microscale positioning. Singer instruments manufactures a mechanical micromanipulator with a 3D pantograph design (Robert 1951); the user holds a pencil-grip stylus and, through the pantograph mechanism, manipulates a probe with a 4:1 reduction of hand motions.

Motorized micromanipulators employ a combination of precision stepper motors and worm gear mechanisms to achieve microscale positioning and motion. These have the advantage of being able to be operated remotely and, in some cases, can be programmed for autonomous operation. A variety of input mechanisms can be utilized, including joysticks and rotary optical encoders. Motorized manipulators can also be computer controlled.

Integrated Systems In order to achieve reproducible, robust, and reliable particle transfers and processing, a combination of microscopes, micromanipulators, and XY/XYZ stages are required. Motorized micromanipulators and XY stages require bulky power supplies and motor drivers (sometimes one per axis of motion), and microscopes with digital cameras often require desktop computers to operate the imaging software. Such equipment occupies large footprints in cleanrooms with limited space, compromises pristine environments with instrument cooling fans, and leads to unsightly tangled masses of cables and wires. The 
NASA Curation Office has recently obtained an integrated system that includes dual motorized micromanipulators, a motorized XYZ stage, and high-resolution digital microscope. The MicroSupport AxisPro system utilizes a graphic user interface control system, allowing all electromechanical components to be operated independently or simultaneously via computer mouse. A number of manipulation and sampling tools are available for the AxisPro, including an ultrasonic milling tool and a device that enables the electrical actuation of stainless-steel tweezers. The compact, integrated design of the system enables the possibility of placing the AxisPro in a $\mathrm{N}_{2}$ sample cabinet with an operator performing sample processing activities remotely. So far, the AxisPro system has been used extensively for microsample handling technique development (e.g. implanting and extracting particles into polyurethane foam collectors).

In order to minimize the risk of sample contamination (especially for collections that have been returned from extraterrestrial sources via spacecraft), materials restrictions are placed on tools and equipment used in sample processing cabinets.

JAXA manipulates samples within its Hayabusa processing cabinet using an integrated mechanical manipulation system manufactured by Hitachi (Yada et al. 2014). The system consists of an XYZ stage, a left and right micromanipulator, and a sealed digital camera; the system is constructed from T6061 aluminum, 304/316 stainless steel, Teflon, and quartz. No lubrication is used for the bearings, and the entire manipulator is disassembled and serviced annually to maintain performance.

Six-Axis Compact Robot Arms While 3-axis micromanipulators have been extremely successful for activities involving the transfer of isolated particles in the 5-20 $\mu \mathrm{m}$ range (e.g. from microscope slide to epoxy bullet tip, beryllium SEM disk), their limited ranges of motion and lack of yaw, pitch, and roll degrees of freedom restrict their utility in other applications. For instance, curation personnel removing particles from cosmic dust collectors by hand often employ scooping and rotating motions to successfully free trapped particles from the silicone oil coatings. Similar scooping and rotating motions are also employed when isolating a specific particle of interest from an aliquot of crushed meteorite. While cosmic dust curators routinely perform with these kinds of manipulations using handheld tools, operator fatigue limits the number of particles that can be removed during a given extraction session. The challenges for curation of small particles will be exacerbated by mission requirements that samples be processed in $\mathrm{N}_{2}$ sample cabinets. We have been investigating the use of compact robot arms to facilitate sample handling within gloveboxes. Six-axis robot arms potentially have applications beyond small particle manipulation. For instance, future sample return missions may involve biologically sensitive astromaterials that can be easily compromised by physical interaction with a curator; other potential future returned samples may require cryogenic curation (Calaway and Allen 2013). Robot arms may be combined with high resolution cameras within a sample cabinet and controlled remotely by curation personnel. Sophisticated robot arm and hand combination systems can be programmed to mimic the movements of a curator wearing a data glove; successful implementation of such a system may ultimately allow a curator to virtually operate in a nitrogen, cryogenic, or biologically sensitive environment with dexterity comparable to that of a curator physically handling samples in a glovebox.

\subsubsection{Methods for Mitigating Triboelectric Charging}

Developing tools and methods for mitigating the effects of triboelectric charging during small particle processing activities is a major objective of microscale advanced curation 
research. Triboelectric charging results from contact, separation, and frictional charge induction (Matsusaka et al. 2010). Examples include friction between storage substrates and instrument support stages or friction between manipulation tools and particles. Many storage substrates currently in use for small particle curation are fabricated out of glass, quartz, corundum, or other optically transparent material that enables the utilization of transmitted illumination; for example, interplanetary dust particles have traditionally been stored and distributed to investigators between a flat glass slide and a glass concavity slide. However, most transparent materials possess poor electron mobility, and any local accumulated charges are unable to easily redistribute. We have identified friction between these slides, particles, manipulation tools, and instrument support stages as a major source of sample electrification. In cases where substrate transparency is not a curation requirement, the glass support slide may be replaced with a silicon wafer. Particles retain a high level of visibility on such substrates (especially under coaxial illumination), and triboelectric charging is significantly reduced such that particles between 40 and $100 \mu \mathrm{m}$ can be reliably manipulated and arranged in arrays without additional charge-mitigation devices. Recently, we have experimented with producing storage receptacles in silicon using focused ion beam (FIB) milling (Fig. 6). We used an FEI Quanta 3D-FEG Focused Ion Beam (FIB) to mill several shallow $(<20 \mu \mathrm{m})$ depressions between $30 \mu \mathrm{m}^{2}$ and $80 \mu \mathrm{m}^{2}$ into the surface of a silicon chip; material was sputtered using a $65 \mathrm{nA} \mathrm{Ga}^{+}$beam at $30 \mathrm{kV}$. A $10 \mu \mathrm{m}$ particle of CM2 meteorite was placed into one of the FIB-produced wells using a pantograph mechanical micromanipulator. The charge-dissipative nature of the Si chip enabled us to successfully acquire a secondary electron image of the stored particle using a $190 \mathrm{pA}$ beam current at $10 \mathrm{kV}$. Storage substrates that also enable electron beam imaging and characterization are desirable, as they minimize the need for high-risk microscale particle transfers between storage and analysis substrates. We are currently investigating this technique to produce storage wells in other charge-dissipative substrates that could enable in-situ elemental analyses.

For instances where the use of transparent substrates is unavoidable, other steps may be taken to minimize frictional contact between insulating materials. For instance, we have constructed slide support frames out of conducting and electrically grounded materials (e.g. miniature aluminum extrusion framing systems). One effective method for minimizing triboelectric charging effects is to restrict small particle processing to times when the ambient humidity is above 60\% (Guardiola et al. 1995); however, this method is not viable for microscale samples that are processed in dry $\mathrm{GN}_{2}$ sample cabinets.

Another extremely effective method for mitigating triboelectric charging effects is the use of a ${ }^{210}$ Po alpha ionizing source. Companies such as $\mathrm{NRD}^{\circledR}$ manufacture commercially available devices designed to reduce static charge via alpha particle emission. Because alpha particles have a short penetration range in air, the sources are most effective when placed within $25 \mathrm{~mm}$ of the sample. Tools, substrates, and samples can be periodically exposed to the Po-210 source as sample electrification is observed to worsen; alternatively, if working distance permits, the source can be left in place during particle transfer operations to remove any transient charges as they are produced. Due to the short half-life of Po-210 (138 days), sources must be replaced annually to remain effective. Also, the use of radioactive sources may be prohibited in certain facilities and typically requires specific safety training and security protocols.

JAXA has developed an electrostatically controlled particle manipulation system to handle Itokawa particles in an ultrapure $\mathrm{GN}_{2}$ sample cabinet (Yada et al. 2014). Instead of attempting to neutralize the charge that has accumulated on the particle, they use it to an advantage by attracting the particle with an oppositely charged needle. The system utilizes a quartz needle with an embedded platinum wire; the samples rest on a grounded conductive 

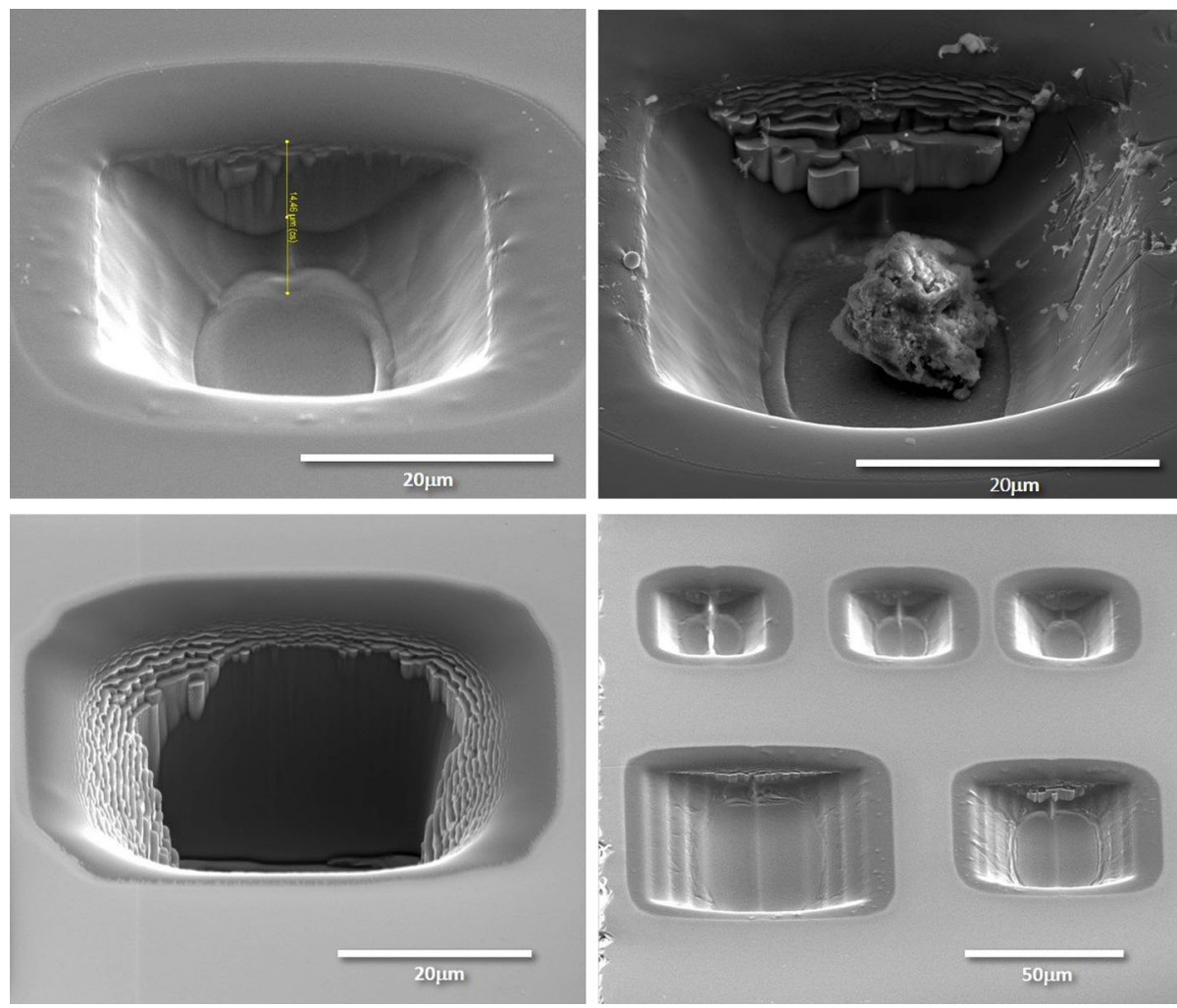

Fig. 6 Secondary electron image of Focus Ion Beam (FIB)-produced wells in Si chip for particle storage and manipulation

surface. When voltage is applied to the system, a charge is induced to the needle by applying a voltage to the platinum wire; this charge is used to attract and release particles and transfer them to custom gold SEM mounts for characterization or to storage wells in quartz slides for allocation and archiving. The NASA Curation Office has reproduced the electromagnetic manipulation system (using needles fabricated at JAXA as part of an ongoing international collaboration between NASA and JAXA curation facilities) and is currently investigating applications for the system for its microscale particle collections.

The tools and methods described here represent only a fraction of the techniques and instrumentation currently utilized and under development for microscale astromaterials sample processing and analysis. An international collective of curators and small-particle scientists at curation facilities, research institutions, and commercial industries continue to collaborate to improve our ability to extract high-quality science from these valuable and unique micro-sized samples.

\subsection{Advanced Precision Cleaning for Storing and Handling Astromaterials}

Precision cleaning of isolation chambers (e.g., gloveboxes and desiccator cabinets), sample containers, and processing tools is important for mitigating terrestrial cross-contamination to pristine astromaterials. Once samples arrive on Earth, the sample environment and how 
it will be handled will begin to alter the pristine nature of the sample. As stated before, the term "precision cleaning" simply means cleaning materials to a prescribed level of cleanliness, which is measured and verified. Aerospace, semiconductor, pharmaceutical, and optics industries are historically concerned with precision cleaning. Standards for precision cleaning are widespread across industrial processes through trade organizations like the Institute of Environmental Sciences and Technology (IEST) (https://www.iest.org), ASTM International (formerly American Society for Testing and Materials; https://www.astm.org), SEMI (https://www.semi.org) and others. Since curatorial precision cleaning does not directly align with a single industry's cleaning standard, curation precision cleaning procedures and protocols for handling astromaterials are derived from many of these established industry standards. NASA also has its own flight hardware precision cleaning standards and are often different and dependent on program and mission. For the NASA Curation Office, precision cleaning standards were mainly derived from the Apollo program and early cleaning recipes and history have been discussed in Calaway et al. (2014).

Currently, precision cleaning at the NASA Curation Office is divided into three categories: PreClean, Final Clean, and Advanced Clean. PreClean is considered gross cleaning when parts arrive from fabrication/machining and/or procurements from a vendor. Final Clean is typically linked to the use of a final cleaning agent, drying, and packaging of the part. During Final Clean, the hardware cleanliness is also measured and verified to meet a certain standard of cleanliness for use. Advanced Clean is a term used for non-routine avenues of cleaning and/or testing of new cleaning methods and techniques. Advanced cleaning is typically done after a routine PreClean and Final Clean process has been completed. This might include techniques that require advanced particulate removal, organic-free cleaning or sterility. Advanced Clean may also use standard or advanced cleanliness verification processes to assess surface cleanliness using a variety of state-of-the-art instrumentation.

In the NASA Curation Office, PreClean and Final Clean support all collections by cleaning the sample processing tools and containers. However, each collection has its own tools and containers, which are cleaned in entirely separate cleaning sessions. All hardware items are put away with the exception of items from the collection being cleaned. The cleaning tanks are cleaned and refilled before starting a new collection. All of this careful effort is taken to mitigate the potential for cross-contamination between the different astromaterials collections. Before attempting gross cleaning, it is important to understand and verify the cleaning chemical compatibility with the material that is to be cleaned. In addition, complex equipment and tools are routinely disassembled, then cleaned and then reassembled in a cleanroom after the precision cleaning is completed. PreClean typically consists of removing any visible grease, dirt, adhesives, or other marks with the use of polyester wipes saturated with isopropanol alcohol (IPA) (70\% IPA and 30\% UPW), if compatible with IPA. If IPA wiping does not work or is not compatible with the material, other cleaners that will not contaminate the material will be used to remove the visible dirt (e.g., citrus-based solvents to remove silicones, ammonia-based solutions, hexane, and household dish liquid have been used for initial gross cleaning). In addition, mechanical gross cleaning may also be necessary in conjunction with cleaning chemicals, such as razor blades and scrubbers (e.g., Scotch Brite pads and nylon brushes). After all visible material is gone, PreClean uses a gross degreasing procedure to remove any machining oils and grease from manufacturing. This is done by soaking and sometimes sonicating the part in a degreasing detergent or chemical. Brulin 815GD (at 5 to $30 \%$ concentration with UPW) is commonly used for stainless steel, aluminum, and titanium metal parts. Freon 113 replacements are also sometimes used for degreasing; for example Honeywell Solstice Precision Fluid, DuPont Vertrel 
specialty fluids (e.g., Vertrel XF), or 3M HFE 7100-DL. Dilute nitric acid is also routinely used to remove trace metal contaminants, such as lead, from newly fabricated items. After degreasing, the hardware is then cleaned with a surfactant. Mechanical scrubbing with polyester wipes or soft brushes are used in a surfactant bath and then placed into a sonication bath for 5 to 15 minutes. Afterwards, parts are removed and spray rinsed with UPW and air dried.

Final Cleaning of equipment and tools are typically centered on high purity chemicals or cleaning agents. Final Clean also incorporates a verification step to evaluate the cleanliness of the part and certify the level of cleanliness and qualification for use. From 1966 to 1994, the final cleaning agent at JSC was Freon 113. Established during Apollo, Freon 113 was an excellent degreaser and final cleaning solvent. The United States government environmental policies on ozone depleting chemicals phased out chlorofluorocarbon production from 1992-1995 which forced the NASA Curation Office to change degreasers and final cleaning agent. After an in-depth research process, the final cleaning agent was changed to UPW in 1994 and is used currently (see Sect. 2.2.2 for details on current UPW purity and system). Final Clean can sometimes redo surfactant cleaning or use of a pre-degreaser, however, most of the time, parts are rinsed with UPW and placed into a UPW cascade bath and sonicated for 5 to 15 minutes. The UPW is often heated to 40 to $70{ }^{\circ} \mathrm{C}$ to provide better cleaning. $\mathrm{GN}_{2}$ is also used during the end of sonication to remove particulates out of the bath. Parts are then removed from the bath and thoroughly spray rinsed with UPW. If another high purity chemical is used (such as IPA or a Freon 113 replacement), this would be applied at this stage, then spray rinsed again with UPW and dried by $\mathrm{GN}_{2}$ (sometimes heated $\mathrm{GN}_{2}$ ) to remove all visible water. During the final rinse, run-off aliquots of UPW may be taken for optical particle counts, liquid particle counts, or TOC analyses. It should be noted that traditional non-volatile residue (NVR) mass balance measurements and black-lights used to be used, however, these methods were eliminated since the Final Clean often showed cleanliness below detection limit of those techniques. The part is then either left to continue to air dry or placed into an oven to remove any more water. After drying, parts are triple bagged in FEP Teflon or nylon bags depending on the collection and its material restrictions. Before bagging, precision cleaning verification often entails an optical inspection of the parts. If parts are shown not to be cleaned to the specified cleanliness standard, parts are sent through the process again.

In the NASA Curation Office, the verification of cleanliness reference standard is frequently IEST-STD-CC1246E, Product Cleanliness Levels-Applications, Requirements, and Determination (IEST-STD-CC1246E 2013). This is a derivative of the historical military discontinued standard MIL-STD-1246 (MIL-STD-1246C 1994). It is in IEST-STDCC1246E that hardware surface cleanliness levels are specified per unit measure both for particles and non-volatile residue (NVR). Particle counts are measured by optical microscopy and/or liquid particle counts. Most NASA astromaterials collections use Level 50 cleanliness standard from IEST-STD-CC1246E. However, the Genesis collection has a dedicated precision cleaning lab, and hardware is generally cleaned to Level 25. For example, cleaning for flight of the Genesis mission involved most surfaces being cleaned to level 25 (no particles $>50 \mu \mathrm{m} / 0.1 \mathrm{~m}^{2}$ ) for particulates per surface area (a function of particle abundance vs. particle size). NVR is traditional measured by means of gravity mass calculation (a as function of mass vs. surface area) but are often limited to technique sensitivity. As an example, R1E-4 is a designation for NVR indicating $<100 \mathrm{ng} / 0.1 \mathrm{~m}^{2}$. More recent techniques have relied on bench-top total organic carbon (TOC) analyzers (as a function of ppb vs. surface area) and more time-consuming analytical instrumentation. This standard is useful because it is frequently cited when cleaning hardware for spaceflight, including sample 


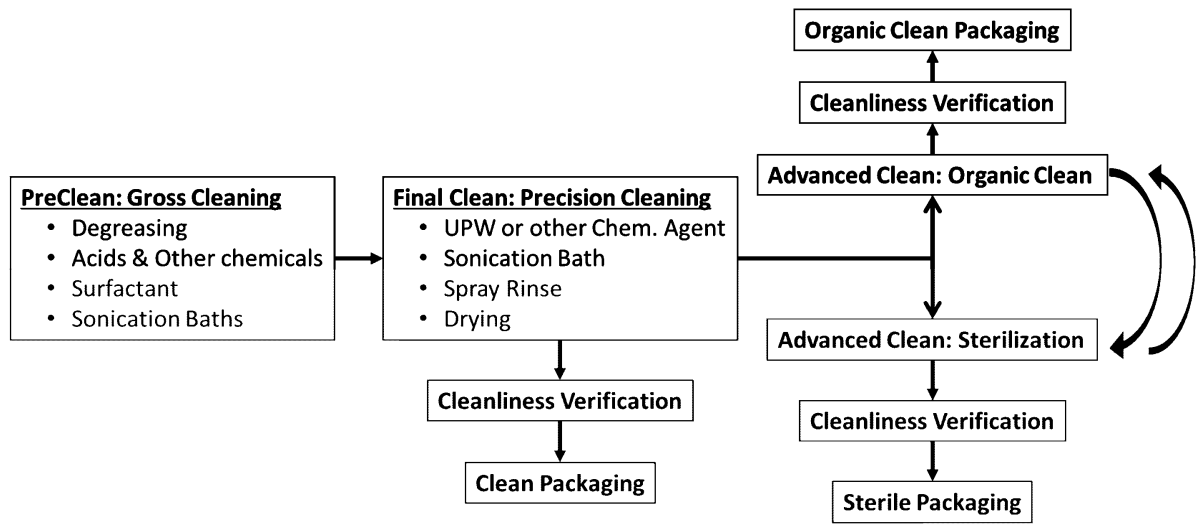

Fig. 7 Basic cleaning process for hardware and tools used in astromaterials curation laboratories. Items to be cleaned are first introduced to a PreClean process that is configured for gross cleaning. Afterwards, items are introduced to a Final Clean process where they are precision cleaned, cleanliness level verified, and packaged for use in the lab. If specialized advanced cleaning is desired, the item(s) are further processed after the routine Final Clean process. This diagram shows the hypothetical process path for organic and sterilization of hardware and tools. These processes could be a single advanced cleaning process or a combination of several advanced cleaning processes. In addition, cleanliness verification can occur at multiple points during the cleaning process or after the process is completed before packaging. Hardware and tools, coupons, and/or final cleaning agent aliquot are commonly used to verify cleanliness to a set standard

collection devices on spacecraft. However, new missions are generally citing total organic carbon (TOC) and the TOC is not always transferable to the NVR level.

\subsubsection{Technical Tensions Among Cleaning for Particulate, Molecular Organics, and Sterility}

UPW cleaning to remove inorganic particles is a mature process for curation of astromaterials. However, the expanding diversity of requirements for high level organic cleaning and sterility invokes conflicts among cleaning techniques that need to be managed. Management options for reconciling these tensions requires attention to detail, perhaps a complex handling sequence, or subdivision of samples into separate handling tracks such as organiccleaned vs. metal-free (e.g. metal vs. plastic handling tools and containers).

A simple model of a cleaning process line (Fig. 7) starts with pre-cleaning followed by UPW cleaning for particle removal. This particle-cleaned product is suitable for inorganic usage or for further cleaning. The product could be further cleaned to high organic cleanliness or to sterility by separate tracks. Selection of a direct separate track may be most efficient and adequate. Greater cleanliness or sterility might be achieved by cleaning for both low organics and sterility. However, due to conflicts in materials, environment, process chemicals, and packaging for high level organic cleaning and sterility, adaptability for specific situations is required.

The revived need for both high-level organic cleanliness and sterility, driven by recent missions such as OSIRIS-REx, Hayabusa2, and possibly Mars Sample Return, have resulted in investigations into advanced techniques and methods for cleaning and for verification of cleanliness. Traditional methods to achieve organic cleanliness, such as solvent extraction and bake-out, can be augmented by UV-ozone cleaning, plasma cleaning, supercritical fluid cleaning, and $\mathrm{CO}_{2}$ SNOW cleaning (Mickelson 2002a, 2002b; Calaway et al. 
2007, 2009; King et al. 2010; Schmeling et al. 2013; Kuhlman et al. 2013). Assessment of surface cleanliness has included the use of instrumentation such as SEM, TEM, FT-IR, Raman, XPS, SIMS, AMF, LC-MS, DART-MS, TD-GC-MS, LA-ICP-MS, and VDP-ICPMS. Protocols to remove adventitious carbon must be followed by surface passivation and extreme control of environment, which includes packaging. Packaging materials must not off-gas organics. Thus, maintaining an organic-clean surface after cleaning is challenging. Traditional heat-sealing will not work for organic samples. For example, the Mars $2020 \mathrm{CK}$ collection has used hermetically-sealing bag clips in-place of traditional heat sealing. Techniques for achieving sterility of containers and tools include standard autoclave, dry heat, UV, hydrogen peroxide vapor and gamma irradiation and electron beam (Allen et al. 1999; Clark 2004). NASA JSC is currently constructing an advanced precision cleaning lab to further study some of these techniques for future missions.

\section{Development of New Astromaterials Acquisition Capabilities on Earth}

The study of astromaterials in the laboratory allows direct analysis of material arising from the full breadth of the history of our Solar System. The continuing advance of technology has improved not only our technological capacity to make measurements but also our ability to minimize contamination and sample modification during collection of freshly fallen meteorites and cosmic dust. Some of these improvements are related to educating the public on proper methods of handling meteorites, but many of the technological advances have focused on improving our ability to track and find materials. In many cases, these improvements result in reduction of exposure time to uncontrolled conditions, which could reduce terrestrial contamination, especially if clean-collection practices are used during recovery operations. Collection of material has been steadily improved by advances in ground-based and satellite sensors, dissemination of information with the growth of the internet and various social media platforms, and especially by the flow of data through freely available data sources. Although the collection of astromaterials may seem, at first, to be a prerequisite of curation, one of the primary goals of advanced curation is aimed at maximizing the science returns of astromaterials samples, and improvements in sample collection techniques has a direct benefit to science. We are in the midst of an exciting period of growth of truly innovative astromaterials sample collection techniques on Earth, and this section illustrates a current snapshot of a rapidly evolving field.

\subsection{New Astromaterials Collection Capabilities for Cosmic Dust}

Since inception in 1981, the NASA Cosmic Dust collection has collected interplanetary dust from Earth's stratosphere using flat-plate, oil-coated collectors. The oil is a high-viscosity silicone (polydimethylsiloxane, $\left.\left(\mathrm{C}_{2} \mathrm{H}_{6} \mathrm{OSi}\right)_{n}\right)$ that is mechanically stiff at collection altitude and temperature but engulfs and protects collected material on return to room temperature. This oil has a long track record of successful recovery of cosmic dust, and recent tests have shown that even oil used for sampling in 1981 retains its original viscosity properties. This is heartening for long-term storage of cosmic dust, but silicone oil has a significant drawback in that it is a contaminant for important studies such as oxygen isotopes, organic species, and amorphous silicates. The community has expressed a desire for at least a subset of samples collected "dry", without the contaminating oil.

Foam collectors are a promising means to accomplishing dry collection. A small number of foam collectors have been flown as a test of concept, which yielded a few cosmic dust 
samples (Messenger et al. 2015). The results were generally acknowledged as positive, and comments to the NASA CD Curator indicate that the scientific community has a strong interest in additional dry collection. To this end, NASA will fly foam collectors as a subset of future collection flights. Collection in foam appears to be straightforward and Messenger et al. (2015) claims that the collection rate is comparable with silicone oil (albeit with a short total collection time for reference), but extraction of collected material from foam is a nontrivial exercise. Foam features relatively deep pits in its surface from which the particles must be extracted. This problem is exacerbated by the observation by Messenger et al. (2015) that "20-50\%" of individual foam cells were broken when observed post-flight, probably by aerodynamic pressure. In order to test identification and extraction methods, NASA Cosmic Dust personnel fabricated an analog foam collector by adhering a $1 / 8^{\prime \prime}$ thick sheet of white polyurethane foam to a surplus Lexan IDP flag using double-stick tape; excess foam was trimmed to match the profile of the flag. Small $(<20 \mathrm{~mm})$ particles of Bells CM2 meteorite were transferred from a concavity slide into individual foam cells using a MicroSupport AxisPro micromanipulation system and bent glass needles. ${ }^{210}$ Po sources were utilized to minimize triboelectric charging effects. Ten particles were implanted into the experimental foam collector apparatus using this technique. The transfer process was then reversed to remove four of the particles from the collector onto cleaned glass slides. None of the particles were lost due to vibration or triboelectric charging effects. Ultimately, foam may be replaced by a more rigid material, but this awaits future work. For the near term, the use of a computer-controlled micromanipulator shows promise in removing material from foam collectors using standard pulled-glass needles.

In addition to dry foam collectors, NASA Cosmic Dust is conducting a project with undergraduate students at Texas A\&M to develop a prototype, high altitude balloonbased dust collection platform. The intent of this new system is to supplement existing aircraft-based collection for two major reasons. One, expanding into a new collection platform adds programmatic depth and resilience to $\mathrm{CD}$ collection efforts. Should the existing ER-2 and/or WB-57 aircraft become unavailable (either temporarily or permanently), dust collection will continue with a balloon-based platform. NASA balloon flights also regularly operate from both the northern and southern hemispheres, opening up the possibility of CD flights intended to collect material from cometary debris streams, which preferentially impinge on the southern hemisphere. The second reason is to offer new ways to improve CD "timed collection" efforts (Dermott and Liou 1994; Messenger 2002). "Timed collection" is collection of material in the stratosphere timed to coincide with the settling time of material sourced from a specific meteor shower and thus with a specific parent body. Collection by aircraft is possible and has been demonstrated (e.g. timed collection of comet Grigg-Skjellerup in 2003 and of comet Giacobini-Zinner in 2012), but significant flight time constraints can impede both the total flight time and timing of "timed collection" attempts. Collection by high-altitude balloons may be more accommodating, as NASA long-duration balloons (LDBs) feature flight times of up to 100 days. Cosmic dust collector(s) could fly on long-duration missions and deploy for collection only during the settling time for material from a specific meteor shower, maximizing collection time at precisely defined intervals to maximize the chances of collecting material from a specific parent body.

A cosmic dust collector prototype for use on high altitude balloons was tested in mid2019 at the NASA balloon research center in Fort Sumner, NM. The prototype is called Cometary and Asteroidal Research of Dust in Near-space Atmospheric Levels (CARDINAL), a name chosen by the students. CARDINAL uses a swing arm with collectors at each end as a low-power means to move air over the collectors, spinning the arm with a small 
electric motor. Collector size, rotation size, and swing arm length were chosen to produce an estimated one cosmic dust particle per day per collector. While this collection rate is about 1/48th that of aircraft-based collection, LDB flights last up to over 100 days as opposed to the typical tens-of-hours collection time of aircraft-based collection. The lower airspeed of balloon-based collection may also be advantageous for collection using collectors that are free of silicone oil, or "dry" collection. CARDINAL seals the swing arm within its body to protect the collectors from contamination, and remains sealed at all times below a set altitude. The lid is movable to expose the collectors at high altitude. CARDINAL draws power from the balloon gondola, and both housekeeping and operations data are stored by the onboard microprocessor for post-flight analysis. CARDINAL is self-contained with respect to communications, with all flight functions programmed prior to flight and no communications needed with a ground station or other external controller. Testing at Fort Sumner revealed significant performance shortcomings, which prevented the first test flight. A second design, which draws heavily on lessons learned from CARDINAL, is currently in early development. This design will simplify the collector encapsulation design, reduce weight considerably, and feature its own battery and solar power module that will perform double duty as flight trim ballast. Ultimately, the intent of this project is to collect cosmic dust during precisely defined periods when specific meteor showers are active, to attempt to collect material from known cometary parent bodies. Both oil-based and dry-foam collectors will be used as part of the continuing development of dry-foam cosmic dust collection.

\subsection{New Astromaterials Collection Capabilities for Meteorites}

Meteorite falls represent opportunities for the recovery of samples that have been briefly exposed to the oxidative, organic-, and moisture-rich environment at the Earth's surface, which is also teeming with life. Substantial contamination of freshly fallen meteorites by such exposure can occur in a matter of days to weeks (Burton et al. 2014; Hilts et al. 2014; Kebukawa et al. 2009). At the same time, advances in curation and contamination knowledge in support of sample return (e.g., Allen et al. 2011; Dworkin et al. 2018; Yada et al. 2014) have resulted in the establishment of curation facilities that prevent or mitigate against such contamination (Herd et al. 2016). Therefore, the present-day challenge is to reduce the amount of time that meteorite samples spend in the field and apply the best methods for their field collection and laboratory curation and handling. The faster the meteorite is recovered and removed to a curation facility, the more scientifically valuable such a sample will be. Rapid collection of meteorites can be seen as strongly complementary to established efforts to collect meteorites from dry deserts such as the Sahara (Grady 2000) and Antarctica (Harvey 2003). These collection efforts produce the large number of meteorites necessary to routinely produce weathered but unusual types such as martian, lunar, ungrouped achondrites, and many others, while rapid collection of fresh falls produces a relatively small number of unweathered meteorites. The combination of both approaches provides a comprehensive approach to meteorite collection, which facilitates study of a wide range of meteorite types with unweathered examples for studying weathering effects, pristine organics and fragile mineral phases (e.g., Haberle and Garvie 2017), and as ground truth for quantifying alteration due to terrestrial weathering.

Significant technological advances have been made in recent years that enable more precise observations and more accurate modeling of meteorite falls, and thus the more rapid potential recovery of the meteorites. Advances fall into two main categories: improved observation of meteor/fireball phenomena, and characterization and modeling of meteorites in dark flight. 


\subsubsection{Fireball Detection and Tracking}

One of the most important "sample return spacecraft" may be the Earth itself. As our planet orbits the Sun, it collects around 40000 tons of extraterrestrial material each year (Flynn et al. 2004; Zolensky et al. 2006a; Zook 2001), ranging from microns in size to occasional large meteorites (and less frequent large impactors). This material originates from a wide range of parent bodies and so has the potential to inform us about the status and histories of a great many parent bodies. Historically, efforts such as the Prairie Fireball Network (Wetherill and Revelle 1981), the Meteorite Recovery and Observation Project (MORP) in Canada (Halliday et al. 1978), the European Fireball Network (Oberst et al. 1998), and others (Bland 2004; Colas et al. 2015; Cooke and Moser 2011; Gritsevich et al. 2014; Hindley and Houlden 1977; Kokhirova and Borovička 2011; Shiba et al. 1997; Sullivan and Klebe 2004; Trigo-Rodriguez et al. 2006; Watson 2009; Weryk et al. 2008; Wiśniewski et al. 2017) surveyed meteors using networks of cameras and recovered small numbers of meteorites. Perhaps the most scientifically significant outcome of these efforts was their recovery of meteorites paired with calculations of their original orbits. This allowed something new in meteoritics-laboratory studies of the recovered meteorites were given a precise "home" in the Solar System, at least immediately prior to the meteorites' fall to Earth. The large number of fireballs observed by these networks and need for accurate fall location calculation encouraged the development of meteor dynamics (e.g., Ceplecha et al. 1998) and strewn field modeling. Today, improvements in digital imagery, geographical information systems, computational capability, and the growing power of the internet to quickly collate data submitted by the public, have driven the development of new fireball reporting networks (Venton 2017). A hallmark of these new networks is their emphasis on including the general public in reporting and other forms of participation (Day et al. 2018a). Another is regular calculation of fireball orbits, such that today the orbits of more than two dozen meteorite falls are known. The Desert Fireball Network (DFN) has used a network of 52 automated digital camera devices to recover four meteorite falls in the period of 2007-2015 (Bland et al. 2012; Sansom et al. 2015). All of the falls are associated with pre-atmosphere orbits, as are a larger number of fireballs that have not yet yielded meteorites. Recent funding from the Australian Research Council expands the Australian-based Desert Fireball Network into a Global Fireball Observatory (GFO), bringing the total area covered by all-sky cameras to over 12 million $\mathrm{km}^{2}$, and enabling 24-hour all-sky observation from both hemispheres with 156 state-of-the-art camera stations. The GFO is expected to see 800 bright fireballs every 6 months and track 5 meteorite falls per month globally.

\subsubsection{Eyewitness Reporting via the Internet}

Historically, most meteorite falls have been recovered based on eyewitness accounts and many of these recoveries were facilitated by eyewitnesses close enough to observe a meteorite striking the ground. Many eyewitnesses doubtlessly observed meteorite falls from a distance, but the fragmented nature of eyewitness reports and lack of widespread public understanding inhibited collection of fallen material. With the growth of the internet, however, several organizations have begun collating eyewitness accounts into meaningful bodies of data capable of rapidly constraining the site of potential meteorite falls. The non-profit American Meteor Society (AMS) stood up a webpage with this purpose in 2006, allowing individuals to log meteor sighting reports at no cost to the user (https://www.amsmeteors.org). The sightings are collated into reports of individual fireballs, and automated calculations, 


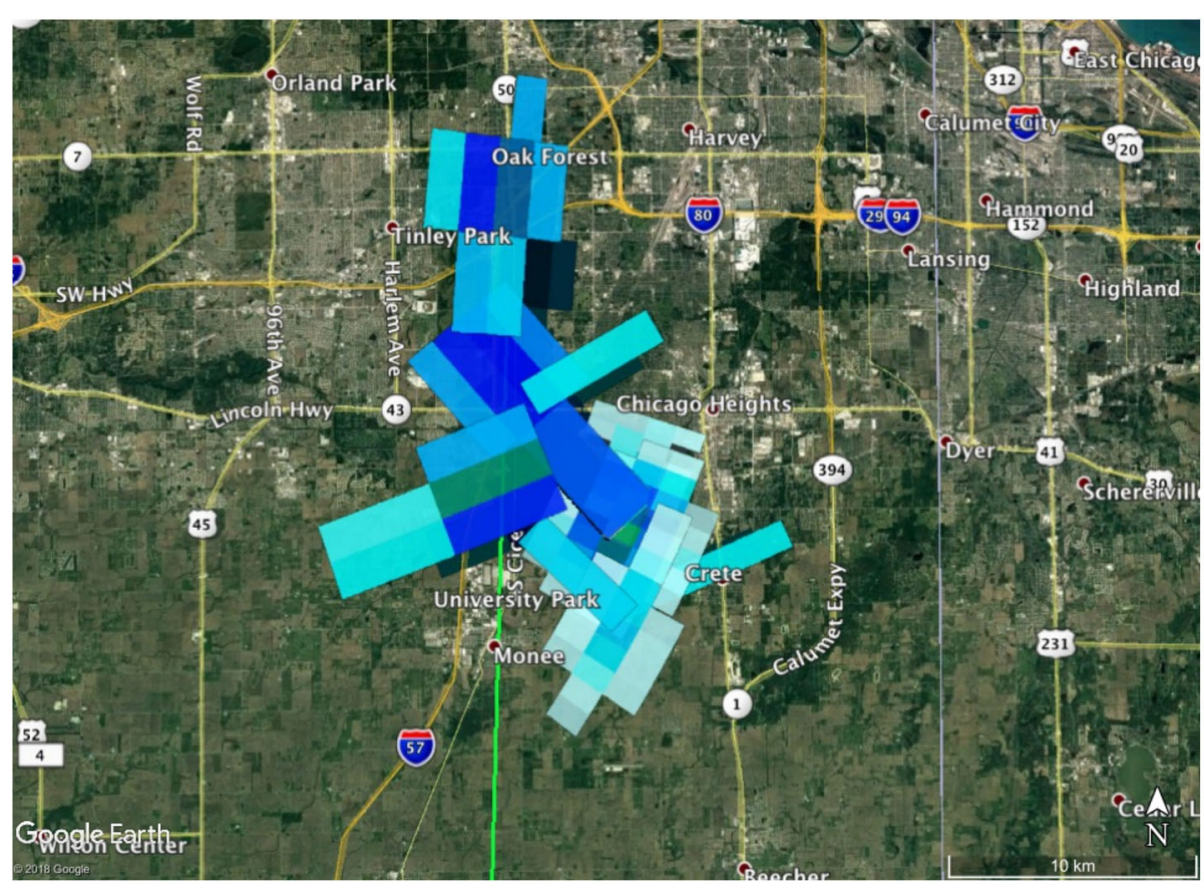

Fig. 8 Top-down composite view of weather radar signatures of falling meteorites. This shows meteorites falling during the Park Forest, IL meteorite fall (26 March 2003). The meteorites are size sorted during free fall to the ground with the most massive stones landing first, and individual radar "sweeps" record a cross-section of the resulting curtain of falling material. Signatures here record meteorites from $15.5 \mathrm{~km}$ down to $5.0 \mathrm{~km}$ altitude, and the altitude range can change for different falls based on the observation geometry of nearby radars. Typical detection timing for a meteorite fall ranges from radar detection of the fireball itself to observation of the last material (larger than dust) to reach the ground $\sim 10-12$ minutes later. Data is provided via website access by NOAA, and this image was composed in Google Earth

based on sighting azimuths, produce an estimated ground track for each event. Similar websites are operated by the International Meteor Organization (IMO) (https://www.imo.net), Fireballs in the Sky in Australia (http://fireballsinthesky.com.au), the UK Meteor Network (https://ukmeteornetwork.co.uk), and EXOSS Citizen Science in Brazil (https://exoss.org). The AMS, for example, recorded 5473 separate fireballs in 2017 and has produced eyewitness-based reports for meteorite falls to include Park Forest, IL (27 March 2003), Battle Mountain, NV (22 August 2012), Sutter's Mill, CA (22 April 2012) and others. With the increase in public access to the internet, these organizations have seen significant growth and can rapidly provide public notice of new meteorite falls (Fig. 8).

\subsubsection{Weather Radar Detection of Meteorite Falls}

Weather radars are commonly used for weather observation and forecasting worldwide, but the cost of building and maintaining a nationwide weather radar system demands that most weather radars are operated as nation-wide, government-run networks. The upside of this is that data is standardized within a national network and many countries maintain archives of their radar imagery. The downside is that public access to weather radar imagery is scant, with most data available in a short-lived image download format, often in the form of a calculated product such as rainfall rate. The United States has, arguably, led the way in free 
public dissemination of weather radar imagery, with the nationwide NEXRAD network operated by the National Oceanic and Atmospheric Administration (NOAA). NEXRAD data are freely available in all formats, from lightly formatted data to compiled images and calculated products (Fig. 9). All NEXRAD data are available online at no cost and are updated in near real time. NEXRAD data are made available on the internet at such a rapid rate that, for a typical meteorite fall lasting 10-15 minutes, at least one set of radar data showing falling meteorites is available online before all of the meteorites have reached the ground. Finding a meteorite fall in weather radar imagery currently requires manual data processing and analysis, but falls have been identified within hours of the event via weather radar imagery (Jenniskens et al. 2012) and the possibility exists for more rapid, automated identification of meteorite falls.

Weather radar detection of meteorite falls was first demonstrated with the Ash Creek, TX fall of 15 Feb 2009 (Fries and Fries 2010). Two researchers, Drs. Marc Fries and Robert Matson, had been investigating weather radar for this purpose without knowledge of the others' efforts and independently noted the ability of NEXRAD radars to detect falling meteorites and provide their fall location with great accuracy. Ash Creek was also noted by a National Weather Service office in Dallas, TX, who serendipitously noted the fall in the imagery of the KFWS radar. Ash Creek was a perfect test case in that it was a sizable fall that occurred in otherwise clear skies and stands out clearly in radar imagery. Since then, twenty-four recovered meteorite falls have been identified in NEXRAD imagery from 1997 to the present, with an additional thirteen identified that have not been recovered for various reasons (Fries et al. 2017). This is an average of 1.8 meteorite falls found per year, with 1.1 per year recovered in the United States and Canada (Fig. 10). Falls are identified by a combination of factors. Falls occur at the time and location identified by eyewitnesses, progress from high altitude to low over a period of 10-15 minutes, may include short-range turbulence caused by supersonic meteorites, and progress from moderate to high spectral width (a measure of the range of sizes of reflectors in a given image pixel) to low spectral width as the meteorites size-sort on the way to the ground. By contrast, nearly everything else that appears in weather radar imagery — such as weather, birds, insects, and aircraft—move laterally and do not show the other features listed above. Work is currently underway to include quantitative measurements of meteorite fall mass, and it may be possible to suggest meteorite types from radar data based on the fragmentation behavior of the bolide.

Weather radars are not limited to the U.S., of course. According to the World Meteorological Organization (a United Nations Specialized Agency), weather radars around the world comprise approximately $3.6 \times$ the total areal coverage of the NOAA NEXRAD network. In other words, if all the world's weather radars could be put to use finding meteorite falls, the number of falls observed on radar could conceivably increase by an additional $\approx 3.6 \times$. Such an increase should provide freshly-fallen meteorites for study and constitutes significant public outreach potential. There are significant obstacles to using weather radar to search for meteorite falls to include (1) some national radar networks choose to limit public access to data, (2) many countries do not archive sufficient data to be useful, and/or (3) some countries use proprietary data formats that are difficult for the public to utilize. These problems must be overcome within individual nations in order to realize the full potential of weather radar data to identify and locate meteorite falls.

\subsubsection{Seismic Data}

Another important asset for locating meteorite falls is seismic data. Seismometers monitor ground motion over most of the Earth's surface, and they are capable of detecting sonic 


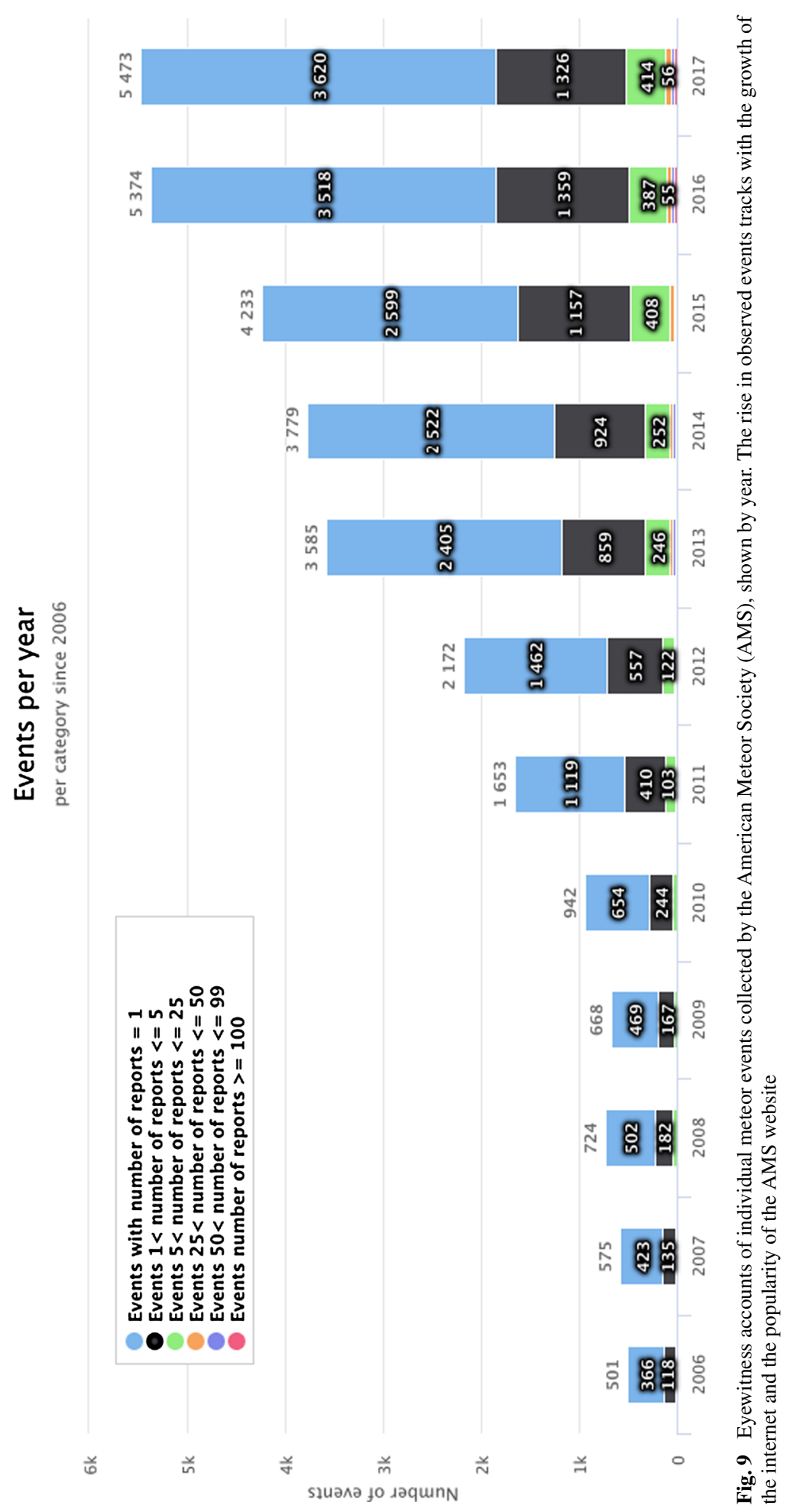




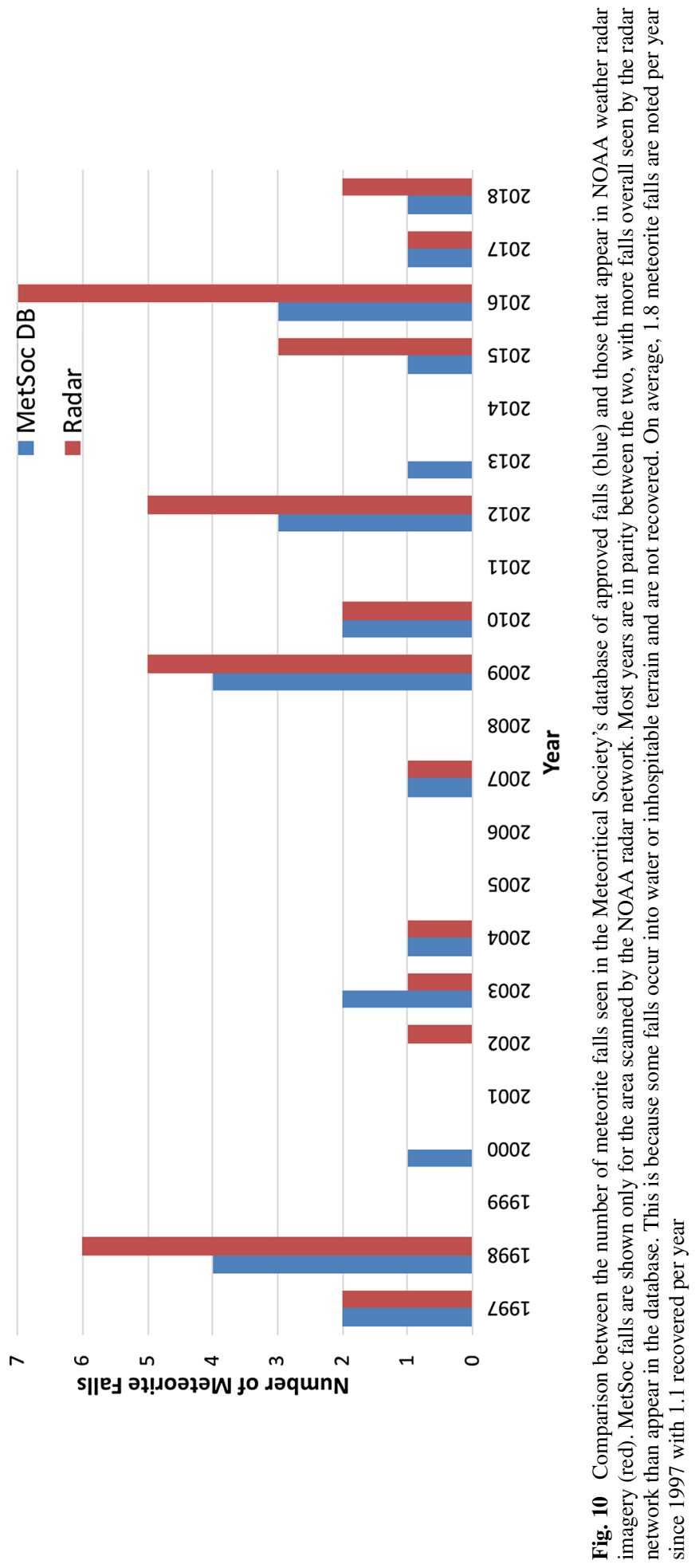




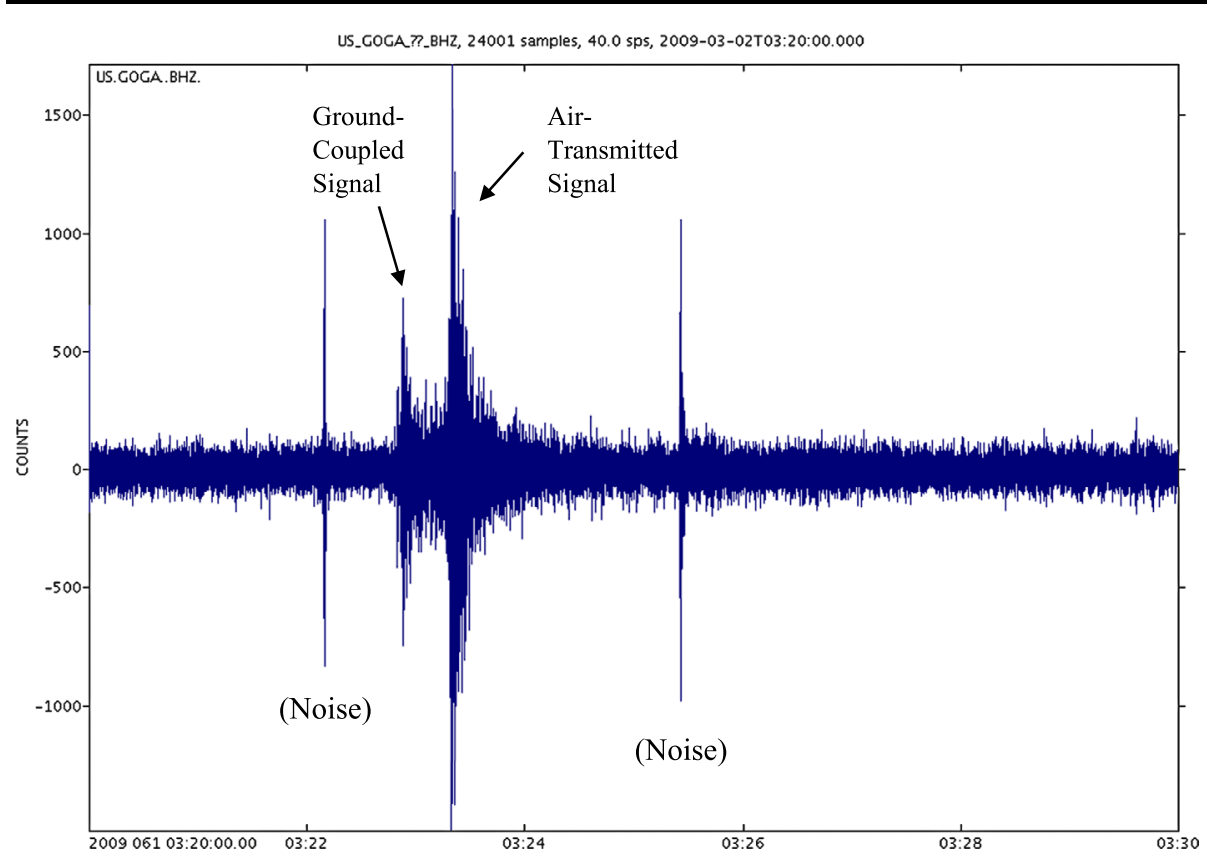

Fig. 11 Seismometer trace showing a sonic boom from a meteorite fall. Seismometers often record a pair of intensity "spikes" like the ones shown here, one from a ground-coupled signal and another transmitted directly through the air (see text). This signature is from the Cartersville meteorite fall (02 Mar 2009), which resulted in a single recovered stone. This illustrates how seismometers provide useful information for even small meteorite falls. Seismometer data provides precise timing of a bolide and can locate the event through triangulation

booms from both high-altitude deceleration of meteoroids (Edwards et al. 2008) and relatively closer low-altitude passage of falling meteorites in "dark flight", or the portion of a meteorite fall after luminous flight. Seismometers are typically operated in networks, and those networks are usually regional or local suites of instruments operated by government entities, universities, or other research facilities. The Incorporated Research Institutions for Seismology (IRIS) database collates publicly-available seismometer data into a common format and makes it available online.

Sonic booms from meteorite falls often appear as a pair of peaks in signal intensity graphs, or seismograms (Fig. 11). The main signature seen in most seismometer data comes from the point of maximum sonic boom generation during the fall, between $\sim 20-30 \mathrm{~km}$ altitude (Ceplecha et al. 1998). The first peak comes from coupling of the air-transmitted pulse with the ground underneath the sonic boom. The resulting ground-transmitted pulse moves approximately $6 \mathrm{~km} / \mathrm{s}$ and outpaces the air-transmitted pulse, usually arriving at the seismometer first. The air-transmitted sonic boom pulse moves approximately $340 \mathrm{~m} / \mathrm{s}$ but is a stronger signal, producing a stronger pulse shortly after the ground-transmitted pulse arrives. Additional sonic booms may arrive afterwards, from individual falling meteorites in dark flight. Such signals are usually less intense than the initial signature. The arrival time of each of these signals is strongly dependent on the distance between the source and the seismometer, permitting triangulation of the source location. 


\subsubsection{Meteorite Recovery from Bodies of Water}

Approximately $70 \%$ of the Earth's surface is covered by water, and that same percentage of meteorite falls disappear into the oceans and other bodies of water. Most of these have historically been lost, although there have been several meteorite falls wherein significant meteorite masses were recovered by people diving after an observed fall. These include Angra dos Reis (the namesake of the angrite clan of meteorites) (Prinz et al. 1977), Peña Blanca Spring where the recovered meteorite landed in a pond in front of a group of ranch hands (Lonsdale 1947), the main mass of Björbole which was recovered from icy water in 1899 (Martin and Mills 1976), and the main mass of Chelyabinsk which was recorded on video landing in a lake (Popova et al. 2013). These were fortuitous recoveries that all feature relatively shallow water and eyewitness(es) who saw the actual point of entry into the water. Recently, attempts have been made to expand this capability by recovering probable meteorite falls from deeper water using Remotely Operated Vehicles (ROVs) and other modern deep-sea technologies.

The Aquarius Project (Bresky and Fries 2018) is a student project to recover meteorites from Lake Michigan. On 06 Feb 2017, a bright green fireball accompanied by sonic booms heralded a large meteorite fall into the lake. The fall was observed by four radars in the NEXRAD national weather radar network. The meteorites now lie on the lake floor at a depth of around 100 meters. A consortium of Lake Michigan-area institutions was formed, including the Field Museum of Chicago, the Shedd Aquarium, and the Adler Planetarium with assistance from NASA and NOAA. Teenage students from Chicago public schools have worked with the project and assist in the design and testing of original devices used to collect meteorites from the lake floor. Aquarius Project updates are archived online (https://openexplorer.nationalgeographic.com/expedition/rovmeteoritehunt). In July of 2018, the students deployed their equipment to attempt to recover meteorites from the lake bed, recovering small rocks and sediment. This material is currently under examination by Aquarius Project institutions, and additional trips to collect material are planned for 2019.

Another attempt to retrieve meteorite material from an observed fall occurred in early July 2018, targeting a very large meteorite fall that occurred in the Pacific Ocean on 07 March 2018 about $20 \mathrm{~km}$ off of the coast of Washington state. Calculations of total meteorite mass based on radar reflectivity indicate that this fall was the most massive fall seen to date by the NEXRAD system since its inception in the mid-1990's. More importantly, the distribution of meteorite mass is unlike any of the two dozen recovered meteorite falls recorded by NEXRAD. The Washington coast fall features significantly more large, surviving meteorites proportional to smaller size fractions than previous events. This implies a stronger than typical mechanical toughness for this fall, which may in turn come from a slow infall velocity or a meteorite type that is inherently stronger than typical ordinary chondrites. The fall itself occurred during region-wide cloud cover and so was not observed directly, although at least two videos record bright flashes through the clouds. It is scientifically important to understand the reason why this fall produced an atypically large number of large meteorites, both as a planetary defense issue and to allow identification of future meteorite falls of the same type from radar data. For these reasons, a one-day effort was mounted by the Ocean Exploration Trust (OET) exploration vessel E/V Nautilus to map the fall site with multibeam sonar and attempt retrieval of meteorite material sufficient to identify the meteorite type. Sonar revealed that the $\sim 100$ m-deep seafloor was flat and featureless. The ROV pair Argus and Hercules performed a seafloor examination/sampling transect along a $\sim 1.6 \mathrm{~km}$ track, collecting one sample with a magnetic rake, five more with a water-jet sediment sampler and one with a scoop. These samples were examined by optical microscopy, Raman spectroscopy, and electron beam analysis at NASA JSC. In June 
2019, a follow-on team returned to the site aboard the research vessel R/V Falkor, operated by the Schmidt Ocean Institute (SOI). This second expedition focused specifically on retrieving $\sim 1 \mathrm{~cm}$-sized meteorite fragments from the area of the meteorite strewn field where that size of meteorite should predominate. Over the course of three continuous days of ROV operations, an SOI-developed pair of sediment samplers sifted a large volume of ocean floor sediment but did not recover $\mathrm{cm}$-sized meteorites. A suite of sediment samples was collected, washed, sieved, and searched in a manner identical to the Nautilus samples, in an iterative search for progressively smaller meteorite fragments. This effort was ultimately successful in the size fractions below $\sim 2 \mathrm{~mm}$ in diameter. At the time of this writing, over 100 small melt spherules and other fragments have been recovered from a combination of the Nautilus and Falkor samples. Work continues on this project to identify any meteorite type(s) among the spherules and whether they can be definitively linked to the fall event on March 07, 2019.

The public outreach aspect of this effort was a dramatic success, with a large global audience watching in real time via the OET webpage as the Argus and Hercules scanned the seafloor and collected samples. Through the course of eight hours with the ROVs on the seafloor, the crew took questions and narrated the effort for a pan-global audience. Overall, both the Aquarius Project and the Washington coast fall recovery show that modern oceanographic surveying and sampling techniques have made water-borne recovery of meteorite falls a real possibility. These pioneering efforts are yielding a first trip up the learning curve towards optimizing the techniques needed, and have set up a powerful new means of engaging students and the public for meteorite research and recovery. At present, the majority of all meteorite falls are lost to science because they fall into the oceans, which cover $\sim 70 \%$ of the Earth. In the future, development of the meteorite recovery techniques explored in this effort could be used to identify and sample meteorite falls for any event that terminates into water. Costs and effort requirements would naturally limit that number, but seaborne meteorite fall recovery could be employed for extraordinary events such as the recent (18 Dec 2018) 173 ktonne-TNT event over the Bering Sea. Another example of a worthy recovery target is the infall of $2019 \mathrm{MO}$, which was observed while still in space and fell into the Caribbean Sea on 22 June 2019.

\subsubsection{The Geostationary Lightning Mapper on GOES Satellites-A New Meteorite Fall Detector}

NOAA recently launched the GOES-16 and -17 satellites, a new design of geostationary weather surveillance satellite. These satellites provide weather imagery services for most of the United States and parts of contiguous countries. For the first time, the GOES-16/17 satellites feature a lightning mapper instrument - the Geostationary Lightning Mapper (GLM). GLM "stares" at a large ground footprint area, collecting imagery at a rate of up to 500 frames/second. The instrument features sufficient dynamic range to detect lightning during local daytime on the ground, and can discern altitude sufficiently to differentiate cloud-toground from cloud-to-cloud lightning. Inadvertently, NOAA built a superb meteorite fall detector with the GLM sensors. While visible and IR-wavelength weather satellite imagery can detect meteorite falls (e.g. Almahata Sitta as described in Borovička and Charvát 2009 and Chelyabinsk as seen by ESA's Meteosat-9 and reported in Miller et al. 2013), considerable luck is required because the image collection rate is very low. Previous GOES satellites, for example, only collected images once every few minutes and a meteor would have to happen exactly at the moment when the camera was operating to be recorded. GLM's rapid imaging and sensitivity to bright flashes renders it a very capable meteor detector, as 


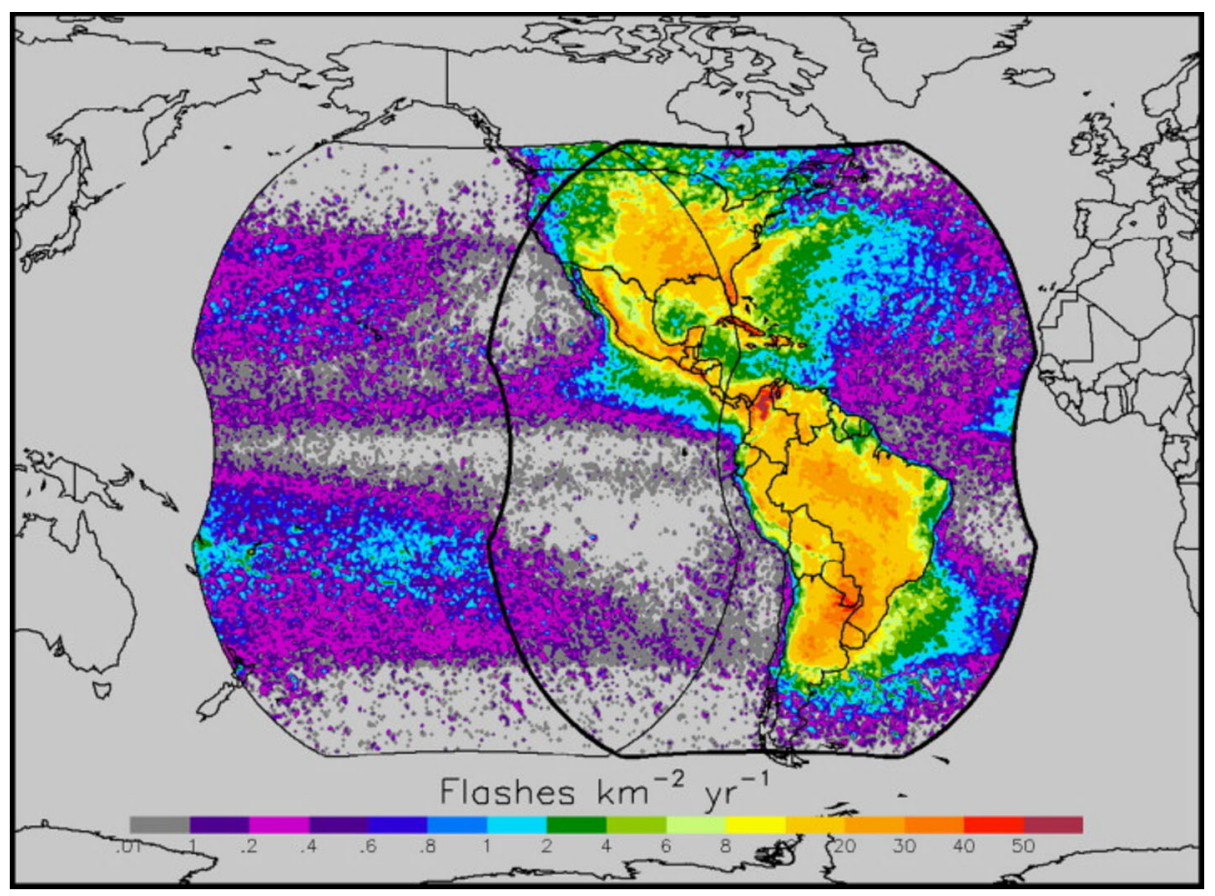

Fig. 12 NOAA graphic indicating the overlapping imaging footprints of GLM sensors on the two GOES satellites, one located off of the US east coast and another off of the west coast. Colors indicate the expected lightning frequency per year. Note that the imaging footprints of the two satellites overlap over most of the contiguous United States and Central America, where stereo imaging of bolides over land is theoretically possible

described recently (Jenniskens et al. 2018). Jenniskens et al. (2018) describe ten separate bolides detected by GLM to include one meteorite fall (British Columbia, Canada, 05 Sep 2017) and has since detected another (Hamburg, MI, 16 Jan 2018). GLM provides location, fireball luminosity, timing, and light curve data for bolides. Presently, both of NOAA's new GOES satellites are in place (GOES-16 and GOES-17) and their GLM sensors are operating and data is available online (https://www.class.noaa.gov). The two satellites cover the continental United States from locations near the east and west coasts, which may allow stereo observation of some meteorite falls (Fig. 12). This feature may be used to rapidly identify bolides among the lightning flashes that GLM is intended to monitor. Lightning usually occurs below $15 \mathrm{~km}$ altitude (e.g., Mecikalski and Carey 2018) while meteors produce optically bright signatures between $\sim 20-90 \mathrm{~km}$ (Ceplecha et al. 1998). Stereo observation of bolides from the two satellites should yield rapid calculations of the altitude of various events, and finding bolides would be a matter of identifying bright flashes that occur at higher altitudes than that of lightning. The possibility exists that streaming GLM data can be used to identify the location, timing, and potential for producing meteorites from bolides in near real-time.

\subsubsection{Putting It All Together-Dedicated Collection of Fresh Meteorite Falls}

The march of technology has availed the astromaterials community with the new technologies and capabilities described here. Historically, even though meteorite falls are far out- 
numbered by meteorite finds, the light terrestrial alteration inherent in recent falls makes them highly scientifically valuable. Regular and rapid collection of freshly fallen meteorites is possible to an extent that a new possibility exists-dedicated recovery of freshly fallen meteorites by a dedicated recovery team. The nature of such a team can take many forms, to include enlisting the assistance of the general public. This approach takes advantage of the spirit of public inclusivity, which created such endeavors as the American Meteor Society's eyewitness reporting program and is a powerful opportunity for outreach. A nationwide program could stand up to mobilize meteorite falls as they are detected, using eyewitness accounts and GLM to identify a new meteorite fall, seismic and radar data to calculate the strewn field, and astromaterials experts encouraging and guiding meteorite recovery at the fall site. Impromptu public lectures and media contact shortly after a meteorite fall have a history of enthusiastic reception from the public and tend to promote recovery of meteorites. The possibility exists that a dedicated collection(s) of freshly fallen meteorites could be founded and sustained in this way.

\section{Importance of Contamination Knowledge Strategies for Sample Return Missions to Maximize Science Returns from Samples}

The scientific value of the returned samples for all previous sample return missions have benefitted from having an archive of contamination knowledge (CK) materials that could include (1) spacecraft hardware, spares, and flight-like coupons, (2) materials used in the fabrication of spacecraft hardware or construction of a curation lab, and (3) witness materials deployed during ATLO or during construction of the curation lab. The information gained from studying the collected reference materials and witness plates is defined as the CK of a sample collection, and the $\mathrm{CK}$ is crucial for verifying and validating scientific results. As part of a sample return mission, these CK samples are archived along with the returned samples and the CK samples are made available for allocation and analysis by the scientific stakeholders of a sample collection. These flown, flight-like, and non-flight reference materials and witness plates provide the scientific community investigating astromaterials with the fundamental ability to reconstruct the contamination history of a sample collection. Furthermore, they serve as a baseline from which to compare tantalizing results attained from the analysis of astromaterials. CK collections are a requirement for sample return missions because contamination control efforts cannot anticipate all possible contamination vectors that can occur during the dynamic activity that is a sample return mission. In fact, all sample return missions have developed non-nominal contamination to some degree, which are highlighted in this section as important lessons learned. In every case, the CK samples have helped to mitigate these unplanned contamination events, improving and, in some cases, enabling scientific returns on the returned astromaterials. The NASA Curation Office has allocated hundreds of samples to scientists in support of gaining contamination knowledge for their respective collections (mainly Apollo, Genesis, and Stardust, and a newly constructed OSIRIS-REx collection). We outline the CK methodology and lessons learned for many completed and ongoing sample return missions and provide insights into best practices for collecting CK when biological contamination from indigenous or exogenous extant life is a possibility.

\subsection{Apollo Program}

For Apollo, contamination knowledge has a broader definition and overlaps with contamination control, compared with recent sample return missions, principally because Apollo was 
a series of missions in which in-lab monitoring of returned samples and hardware resulted in improvements for subsequent missions. Here we cite a 1965 formal report wherein three scientific discipline groups made early recommendations concerning sample collecting materials and procedures relevant to contamination. This is followed by examples of organic and inorganic contamination of samples being measured, by both curatorial staff and by researchers using samples, which resulted in improvements both to lunar surface sampling procedures and hardware and curation handling practices. Thus, contamination knowledge is an ongoing process for returned sample collections.

Early advice on contamination issues was captured through 3 of the 7 discipline working groups convened in 1965 for the purpose of advising NASA on science for a 10-year period (NASA 1965). Geochemistry, Bioscience, and Geology Group reports were most concerned with contamination knowledge. For example, top level equipment requirements included: (1) "Sample containers should keep samples sterile and chemically clean. Stainless steel is acceptable. More studies should be completed relative to the use of Teflon in the lunar environment." It was the Geochemistry Group that emphasized the value of sample analysis and foresaw many of the curation guidelines that are still followed today. The report calls for CK studies to determine the amounts and effects of outgassing of the astronaut suits, the escape of atmosphere from the Lunar Excursion Module (LEM), analyses of possible contaminants in LEM fuel and effects of those contaminants on samples. The Geochemistry Group specified acceptable materials to touch the samples (materials that would not interfere with scientific measurements). In general, this meant use of materials of known and simple chemistry, easily distinguishable from lunar material.

\subsubsection{Apollo Organic Contamination Knowledge}

Apollo organic contamination monitoring conducted in the Lunar Receiving Laboratory (LRL) offers an opportunity to compare the monitoring effort on sample handling equipment to the actual detection of organic compounds in lunar samples by investigators. Apollo planners conducted extensive organic contamination monitoring of the containers, tools, and sample handling facilities. Simoneit et al. (1973) summarized the potential sources of organic contamination: (1) surface contamination of the lunar-bound rock box and its contents; (2) surface contamination on the Apollo lunar hand tools used to obtain samples on the lunar surface; (3) exhaust products from the lunar descent engine and reaction control system engines (both using unsymmetrical dimethyl hydrazine and nitrogen tetraoxide); (4) lunar module outgassing; (5) astronaut spacesuit leakage; (6) particulate material abraded from spacesuits or other sources during EVA; (7) venting of lunar module fuel and oxidizer tanks, cabin, and waste systems; (8) venting of spacesuit life support back packs; (9) exposure to LRL vacuum or nitrogen processing chambers; (10) surface contamination of sample processing tools and containers; (11) surface contamination of containers sent to PIs. Items 1, 2, 3, 9, 10, and 11 were considered most serious. Simulations, modeling, and engineering data were used to estimate the contamination contributed by flight items 3, 4, 5, 7, and 8 (Aronowitz et al. 1966a, 1966b). Virtually all rocket exhaust products were low molecular weight and rapidly diffused over large areas. Because of their low concentration, this was not predicted to be a major contaminant. The varied organic products included acetylene, HCN, ethylene, formaldehyde, methyl amines, and others.

For laboratory handling operations, measurements of contamination via "monitors" or witness plates were used. Clean coupons of a woven aluminum alloy called York mesh (2024 aluminum alloy) or aluminum foil were processed along with the lunar-bound tools or placed inside the rock boxes bound for the Moon. Upon return, these coupons were analyzed by solvent extraction and subsequent gas chromatography and mass spectrometry. 
Aliquots of clean Ottawa sand, exposed inside sample processing cabinets, were analyzed by direct pyrolysis and mass spectrometry. The solvent rinsings from tool, container, and cabinet cleaning were also analyzed. Some of the most frequently encountered contaminants were hydrocarbons from pump oils and fatty acids. Detected in the vacuum chamber, some of the fatty acids were thought to be from the polishing compound used on the rock boxes. Dioctylphthalate, a common plasticizer additive for polyethylene, was ubiquitous in cabinets and bags. Simoneit and Flory (1971), Flory and Simoneit (1972), and Simoneit et al. (1973) provide an extensive list.

York mesh and aluminum foil monitored organic contamination levels of about $1 \mu \mathrm{g} / \mathrm{cm}^{2}$ inside the rock boxes. Bakeout of the Apollo 11 rock box actually added organic contamination, but as a result of the monitoring, cleaning improvements were made which produced flight hardware for Apollo 12 through Apollo 15 with only 10-100 ng/ $\mathrm{cm}^{2}$ contamination (Simoneit et al. 1973). With exceptional care, curatorial cleaning procedures during Apollo could produce $1-10 \mathrm{ng} / \mathrm{cm}^{2}$ contamination ranges for polished, planar surfaces. Flory and Simoneit (1972) concluded that organic contamination to lunar samples during Apollo 11 was in the $1 \mu \mathrm{g} / \mathrm{g}$ (ppm) range, but improved to $0.1 \mu \mathrm{g} / \mathrm{g}$ (ppm) for Apollo 12 .

The actual analyses of lunar samples were consistent with the estimated contamination levels. Burlingame et al. (1970) concluded, based on analyses of their allocated samples, existence of systematic organic contamination of about $5 \mu \mathrm{g} / \mathrm{g}$ for Apollo 11 samples, except for those samples processed in the organic reserve cabinet. Reports of organic compounds in lunar fines from other investigators were mixed, ranging from no detection at the $\mathrm{ng} / \mathrm{g}$ levels (Abell et al. 1970; Lipsky et al. 1970; Meinschein et al. 1970) to ng level detection of various organics (Henderson et al. 1971; Murphy et al. 1970; Preti et al. 1971) and 0.5 ppm via pyrolysis (Oro et al. 1970). Porphyrin-like pigments were detected at the trace ng to pg level by Kvenvolden et al. (1970) and Hodgson and co-workers (1970, 1971), but not by Rho et al. (1970, 1971, 1972). Porphyrins as a possible rocket exhaust contaminant were discussed. Amino acids were detected at the $50 \mathrm{ng} / \mathrm{g}$ level by Hare et al. (1970) and Gehrke et al. (1970) after aqueous or other processing to the sub-nanogram level (Murphy et al. 1970) and below detection by Gehrke et al. (1972). No viable organisms were detected in Apollo 11 and 12 samples (Oyama et al. 1970, 1971; Taylor et al. 1971). (For estimates of indigenous lunar carbon in soils and breccias, Vaniman et al. 1991 present values of about $100 \mathrm{ppm}$ selected from analyses less likely, but still possibly, containing terrestrial contamination.) The presence of diamond and polishing compounds on the surfaces of Apollo thin sections have also been documented by Raman spectroscopy, and these contaminants are likely a common result of the thin-section making process (e.g., Steele et al. 2010).

Keeping samples organically clean in the LRL proved difficult. Thus, a small facility to analyze and repackage a lunar sample collected in a special container was constructed at the University of California, Berkeley (Burlingame et al. 1971). The organically clean area consisted of two gloveboxes in tandem preceded by a vacuum entry chamber. The atmospheric nitrogen gas was scrubbed to remove oxygen, water, and organics. The glovebox was equipped with a liquid nitrogen cold finger to remove water generated by the glove operator.

In summary, Apollo organic contaminants were greatly reduced by institution of (1) restrictions on materials allowed contact with, or proximity to samples; (2) isolation of samples in controlled environments; (3) procedures to clean all surfaces in proximity to or contact with samples; and (4) controls on fabrication, processing, and handling of lunar sample hardware. 


\subsubsection{Inorganic Contamination Knowledge}

As is the case with organic contaminants, feedback from investigators on inorganic contaminants was essential in improvement. Some materials were selected for use as lubricants or seals because those materials were not predicted to interfere with scientific measurements. Thus, it made engineering sense to use an alloy of $90 \%$ indium and $10 \%$ silver for the metal knife-edge seal on the Apollo rock boxes. It made engineering sense to use molybdenum disulfide as thread lubricant in curation sample containers. However, these materials were not of sufficient purity for high precision scientific research. Only a few unopened lunar samples remain in indium-silver sealed containers. The use of $\mathrm{MoS}_{2}$ was discontinued and all containers removed from service. Molybdenum disulfide was replaced with a Teflon thread lubricant Xylan. However, Xylan was not pure Teflon and contained a binding agent, so Xylan was also removed.

Attention to detail is required and compromises made, especially for the fabrication processes, and this is illustrated by engineering selection of surface treatments for the Apollo drive tube cores and drill cores. The materials used for fabrication of the large diameter Apollo drive tubes, anodized aluminum, and the drill core tubes, canadized (a proprietary passivation technique) titanium were selected for engineering reasons. A list of materials would not normally raise contamination flags, unless the reviewer understood the details of the process (and in many cases an engineering requirement might be the best solution anyway). Lead content in amounts compromising science results was found in the anodized aluminum and in the canadized titanium by investigators making extremely low level measurements. The solution in the drive tube case was to physically remove the outer $1 \mathrm{~mm}$ of regolith during core dissection, before sample allocation.

One excellent example of institutionalized CK in Apollo sample curation was the construction, completed in 1979, of Building $31 \mathrm{~N}$ at JSC to house the Apollo lunar collection. The entire design and construction was reviewed in real time by a facility subcommittee of the Lunar and Planetary Sample Team. The committee, comprised of planetary petrologists and geochemists - users of the lunar samples for research—requested chemical analysis for most of the material selections and reviewed the data in detail. Examples are chemistry of paint, floor coverings, adhesives, electrical cords, etc.

\subsubsection{Ongoing Contamination Knowledge Efforts}

Collection of CK for the Apollo curation lab continued after construction of the lab and continues to this day. This process is often a joint effort between curation personnel and members of the scientific community that develop in response to interesting, novel, or unexpected results stemming from scientific analysis of the samples. We provide here two notable examples of successful collaboration between curation personnel and the scientific community to better understand the contamination environment within curation facilities at NASA JSC. As mentioned above, Xylan was used on screw threads in the Apollo processing cabinets, tools, and containers to prevent galling. Over time, however, it became evident that the Xylan did not adhere well to the screws because it was flaking off into the processing cabinets and hence served as a potential source of contamination to the Apollo and Antarctic Meteorite samples (Xylan has $45 \mathrm{wt} . \% \mathrm{C}$ and $4 \mathrm{wt} . \% \mathrm{~N}$, and it is not removed by step combustion until samples are heated above $600{ }^{\circ} \mathrm{C}$; Wright et al. 1992). In response to the characterization of Xylan as a potential contaminant that could affect the analysis of $\mathrm{H}, \mathrm{C}, \mathrm{N}$, and $\mathrm{O}$ in astromaterials samples, Xylan was banned from use in any of the curatorial sample-handling hardware at NASA JSC. More recently, a concern was raised 
that the stainless steel tools used during the processing of Apollo samples may contribute highly siderophile elements (HSE) to the processed samples (Papanastassiou et al. 2015; Tikoo et al. 2014), given the low abundances of HSE that occur naturally in lunar samples (Walker et al. 2004). Consequently, the stainless steel tools and sample containers used in the Apollo curation labs were subsampled and analyzed to determine a contamination threshold that would affect HSE analyses of Apollo samples (Day et al. 2018b). Day et al. (2018b) reported that the potential for HSE contamination from the stainless steel containers and tools was low.

\subsection{Genesis}

Genesis was the first sample return mission since the Apollo program and ended a 32 year hiatus for sample return missions. The Genesis mission launched on August 8, 2001 and traveled to the Earth-Sun Lagrange 1 (L1) point. The Genesis spacecraft was held in halo orbit at L1 outside Earth's magnetosphere for 2.3 years, and the mission collected solar wind plasma that was implanted into several arrays of high purity materials and subsequently returned to Earth for analysis. Unfortunately, due to an inversion design mistake of the sample return capsule's (SRC) drogue parachute gravity switch, the SRC experienced a terminal velocity hard landing at the Utah Test and Training Range (UTTR) on September 8, 2004. The impact into the lacustrine sediment breached the science canister and littered thousands of broken high purity collectors throughout the science canister and SRC. Despite this set-back, curation contingency plans were invoked and after much effort decontaminating samples at UTTR and JSC's curation laboratory, all primary science mission goals were achieved and Genesis is marked as a successful mission. The ability of Genesis to rise from the ashes was in-part due to the fact that at mission inception, the mission had a well-orchestrated CK plan that led to a remarkable $\mathrm{CK}$ collection.

Contamination knowledge for Genesis solar wind sample return is captured in archived reference materials and associated documentation of six types, from pre-launch to post landing events: (a) flight-like collector substrates; (b) science canister duplicate components; (c) assembly environment material coupons and process witness plates; (d) post-landing UTTR soil samples; (e) post-landing science canister and sample return capsule hardware; (f) recovery processing tools and containers. Thus, Genesis is an example that contamination knowledge is an ongoing effort and that post-recovery contamination knowledge is important.

\subsubsection{Flight-Like Collector Substrates}

Genesis had ambitious goals for determining the elemental and isotopic composition of the solar nebula. Desired elemental accuracy was $2 \sigma$ limits of $\pm 10 \%$ of the number of atoms $/ \mathrm{cm}^{2}$ on the collector substrates. Desired isotopic precision for many elements was $\pm 1 \%$ compared to terrestrial standards. Given that the estimated 2-year fluence (atoms per $\mathrm{cm}^{2}$ ) for the more abundant elements is in the $10^{8}$ to $10^{12}$ range, requirements for bulk purity and surface cleanliness of collectors were very stringent (Burnett et al. 2003). Much effort was expended by the science team in verifying purity and surface cleanliness of candidate batches of collector substrates. Fifteen types of ultra-pure materials were flown as collector substrates. Three hundred passive collectors, mounted in 5 arrays, individually consisted of single crystal silicon (FZ and CZ), sapphire, germanium, and sapphire coated with aluminum, silicon, diamond-like-carbon, or gold. Targets in a concentrator for $\mathrm{O}, \mathrm{N}$, and $\mathrm{C}$ were comprised of silicon carbide, isotopically enriched polycrystalline diamond and 
diamond-like-carbon coated on silicon. Additionally, special collectors of metallic glass, gold foil, polished aluminum alloy and molybdenum coated foils were deployed (Jurewicz et al. 2003). The diversity of the collector materials on these arrays not only provided multiple analytical background choices for optimum specific analyses, but also multiple material choices for the various surface cleaning processes that the hard landing subsequently required. The samples archived for $\mathrm{CK}$ served as reference pieces for purity and surface cleanliness or may have been implanted to make calibration pieces during analysis. These materials were also widely used to test surface cleaning protocols before cleaning Genesisflown samples. It was evident in some cases of solvent application that reactivity of Genesisflown pieces was different than non-flight reference pieces, presumably due to solar irradiation. To date, allocations of 600 Genesis-flown samples were accompanied by allocations of 300 collector reference materials. The base inventory supporting these allocations is 5000 Genesis-flown collector samples and 2000 non-flown collector reference substrates.

\subsubsection{Science Canister Duplicate Components}

The canister containing the payload of samples for return was cleaned using ultrapure water (> $18 \mathrm{M} \Omega$-cm resistivity) inside of an ISO Class 4 cleanroom. Re-assembly, including installation of ultraclean collectors, was performed by staff completely enclosed in powered HEPA filtered Dryden suits. More than 200 duplicate canister components, with many cleaned exactly like flight components, are archived for comparison of contaminants. Some cleaning fluids and manufacturing fluids (e.g. electric discharge machining oil) were also archived.

\subsubsection{Assembly Environment Material Coupons and Process Witness Plates}

More than 100 environment material coupons of the assembly room are archived. Examples include samples of wall construction material, flooring materials, adhesives, paint, fire retardant and subsamples of air handler intake filters. Process witness plates for particle chemistry and airborne molecular contaminants were periodically set out, but only the data were saved (Allton et al. 2016).

\subsubsection{Post-Landing UTTR Soil Samples}

Just prior to Genesis capsule return, 8 UTTR soil samples from 5 sites were collected by the helicopter recovery crews as they practiced. These samples are archived and have been allocated for contamination studies on solar wind collectors. After the capsule hard landing and recovery of the spacecraft components, eighteen 5-gallon buckets of UTTR soil and spacecraft materials from the impact site were collected and archived. Subsequently, collector fragments were high-graded and removed from buckets for permanent archive.

\subsubsection{Post-Landing Science Canister and Sample Return Capsule Hardware}

Many of the collectors were broken, but most remained confined in the science canister. The field crew was able to gather and transport to a nearby cleanroom the entire science canister containing most of the collectors and the sample return capsule major components within 8 hours of the crash. All of this material is archived for CK, except for pyrotechnic devices and batteries, which were deaccessioned after 5 years (Stansbery and Team 2005). Ellipsometry was used to measure molecular contamination on some of the collector plates (McNamara and Stansbery 2005; Stansbery and McNamara 2005). 


\subsubsection{Recovery Processing Tools and Containers}

Select recovery and UTTR processing tools and containers are archived for CK. Examples include polystyrene containers, fine brushes, and cleanroom post-it paper used for securing small fragments. The post-it adhesive remains under investigation and these CK samples have been helpful. The lesson here is awareness that any material added to the handling stream at the last minute should have batch specific material archived.

\subsection{Long Duration Exposure Facility}

The Long Duration Exposure Facility (LDEF), was a school bus-sized cylindrical facility designed to provide long-term data on low-earth orbit ( $\sim 300$ miles altitude) environment and its effects on space systems, materials, and operations (Kinard and O'Neal 1991). Originally intended to be the cargo for the first space shuttle mission in 1981, it was finally placed in low-Earth orbit by Space Shuttle Challenger in April 1984. Fifty-seven science and technology experiments from nine countries flew on the satellite. The original plan called for the LDEF to be retrieved in March 1985, but because of the destruction of Challenger (the only shuttle with a sufficiently large cargo bay to accommodate LDEF) it was eventually returned to Earth by the newly built Columbia in January 1990. LDEF was an early test bed for ideas on micrometeoroid capture. It was carefully placed in orbit and gravity stabilized such that its orientation relative to Earth remained constant. Thus, the trailing side of the satellite would capture micrometeoroids but no space debris and see no secondary impacts from satellite surfaces - completely eliminating the most significant sources of contamination to captured astromaterials. No subsequent spacecraft has repeated this feat.

Unfortunately, shortly after recovery into the still open Columbia cargo bay the shuttle began to rotate end over end, destroying many experiments and causing severe sample contamination. NASA Mission Control chose not to waken the sleeping astronauts and halt the rotations, despite pleas from the LDEF science team. Thus, recovery of LDEF by the Space Shuttle was non-nominal, resulting in contamination and thereby degrading many mission goals. An important lesson is to carefully consider the possible deleterious consequences of using an astronaut crewed platform for sample recovery operations.

Despite these problems, the LDEF mission was successful in guiding scientists to the design of the capture media for the subsequent Stardust Mission. However, one LDEF lesson was not properly learned. Outgassing of silicone-based adhesives and lubricants coated most of the exterior of the satellite with a Ca- and Si-containing coating, which was baked to a brown color by solar radiation (Whitaker and Dooling 1995). This "brown stain" was to reappear with a vengeance in the Genesis, and to a lesser extent Stardust Missions.

\subsection{Stardust Mission}

For the Stardust Mission, contamination control procedures were integral to flow of spacecraft manufacture, assembly, testing, flight, and recovery, and the science team took a very active role in planning and implementing contamination control measures, monitoring contamination through numerous witness materials (Sandford et al. 2010; Zolensky and Girard 1997). However, despite these precautions, the captured comet Wild 2 coma dust grains experienced significant contamination from several sources, including the presence of indigenous organic and inorganic material in the silica aerogel capture media, spacecraft outgassing, and an unfortunate sample return capsule (SRC) recovery procedure. 


\subsubsection{Preflight Contamination}

The flight aerogel used in Stardust was marveled, but cleaning it was not a sufficiently high priority for the mission. There were alternative sources for the silica aerogel, which were known to be cleaner than the material manufactured by the Jet Propulsion Laboratory in Pasadena, CA. In addition, recommended work on improving aerogel cleanliness were not adequately performed. Synthesis of the aerogel employed a tetraethyl orthosilicate precursor in a solvent that included ethanol, methanol, acetonitrile, and/or other organic liquids, and Synlube 1000 was used as a mold release agent (Sandford et al. 2010). In the end, the severely contaminated aerogel was baked to reduce the volatile organic content, but several weight percent of carbon in the form of organics remained strongly bonded to the aerogel. To be fair, most persons believed at the time that organics could not be adequately captured by aerogel at the mission capture velocity of $6.2 \mathrm{~km} / \mathrm{s}$. The comet Wild 2 coma grains entered the Stardust aerogel at $\sim 6.1 \mathrm{~km} / \mathrm{sec}$. Such collisions are sufficiently energetic that they could alter any organic compounds originally present in both the impacting particles and the aerogel collector material (Sandford et al. 2010; Sandford and Brownlee 2007; Spencer et al. 2009; Spencer and Zare 2007). Thus, it came as a surprise when relatively intact cometary organics were recovered from a few captured coma grains. Had we known this was possible, we would have undoubtedly made greater efforts to fly organically-clean aerogel.

It is now clear that some fraction of the impacting comet Wild 2 coma particles survived with little or no alteration, while other portions of the samples were severely heated (Brownlee et al. 2006; Elsila et al. 2009; Sandford et al. 2006, 2010; Zolensky et al. 2006b). Conversion of carbon original to the aerogel, and in the impacting cometary particles, into new forms likely occurred in a similarly variable manner. Thus, before one can assign organics seen in Stardust samples to a cometary origin, it is necessary to consider the possibility that they are either altered cometary materials or materials formed from carbon original to the aerogel. IR absorption difference-maps of individual tracks suggest that impacting Stardust particles do not convert the majority of the original carbon in the aerogel tiles into new chemical forms that remain in the aerogel. However, laser ablation, laser ionization mass spectrometry $\left(\mathrm{L}^{2} \mathrm{MS}\right)$ studies demonstrate that at least a small amount of the original aliphatic carbon in the aerogel is converted into aromatic materials in the form of lightweight polycyclic aromatic hydrocarbons (PAHs). Thus, while most of the original aerogel carbon appears to be unaffected by the impact process, the issue of the possible presence of impact converted organics must be considered on a case-by-case basis whenever specific organics are being sought in Stardust aerogel samples.

Additional contaminants found their way into the aerogel during flight. These include materials outgassed from nearby spacecraft components, propellant byproducts, and secondary materials from dust impacts on other parts of the spacecraft, particularly the Whipple shields and solar panels. Cometary particles impacted on the aerogel tiles in the collector tray perpendicular to the forward direction. Thus, any tracks seen with oblique orientations must be either due to strikes by random interplanetary dust particles or to secondary materials from impacts on other parts of the spacecraft. Oblique tracks have, in fact, been found in the flight aerogel tiles, most of which fall in non-random spatial distributions on the cometary collector (Westphal et al. 2008). The materials in these tracks could include components from both the original impactor and from the spacecraft. Many of these tracks originated from a grazing impact on the central Whipple shield of the spacecraft as the origin of clustered low-angle oblique tracks. In these tracks, the most likely contaminant would be the Mylar thermal protection material that wrapped the edge of the Whipple shields. A second population of high-angle oblique tracks unambiguously originate from a non-cometary 
impact on the spacecraft bus just forward of the collector. The exact location of this strike on the spacecraft bus is not known, but possible contaminants include materials used for the sides of the spacecraft bus-highly ordered graphite embedded in an epoxy matrix.

In summary, it is clear that the Stardust cometary collector tray was struck by a limited number of secondary particles resulting from impacts on other parts of the spacecraft. Materials in these oblique tracks should be viewed with considerable caution before interpreting their significance as possible cometary materials. Fortunately, the most likely contaminants, Mylar wrap on the Whipple shields and carbon composites from the body of the spacecraft, have distinctive $\mathrm{C}$ X-ray absorption near-edge spectra (XANES) that make them relatively easy to recognize. At present, there is no evidence that this process has introduced contamination outside the domain of the oblique tracks themselves.

\subsubsection{Contamination During Flight}

It is possible that contaminants could have been introduced to the Stardust sampling trays directly from the spacecraft during its nearly 7-year flight. This is of special concern for the aerogel collectors since aerogel, with its very large surface area to mass ratio, is an excellent 'sponge' for adsorbing contaminants. To assess the extent of on-flight contamination, several 'witness coupons' were enclosed in the Stardust SRC (Tsou et al. 2003). These coupons included $1 \mathrm{~cm}$ diameter disks of aluminum and sapphire, and one 'interstellar' aerogel tile ( $2 \mathrm{~cm}$ wide $\times 4 \mathrm{~cm}$ long $\times 1 \mathrm{~cm}$ deep). These coupons were located on the arm that deployed the aerogel collector array and were placed low enough that they resided in the shadow of the main Whipple shield. Thus, these coupons were exposed to the same flight environment as the aerogel collectors for the entire mission, but were never directly exposed to the cometary influx.

Examination of the aerogel witness coupon showed no visible signs of adhering materials or stains. Although we know that silicone-based adhesives and lubricants outgassed during the mission (famously coating the cold camera optic surface), none of the exposed surfaces in the Stardust sample return canister showed any signs of the 'brown stain' seen on many of the surfaces of the LDEF (Fred Hörz, personal communication, 1990) and Genesis hardware (Burnett 2013). Analyses of the aerogel witness coupon (i.e., aerogel that was exposed to all environmental conditions as the collector aerogel except the comet) shows similarities to collector aerogel, although the levels of contaminants, when detected, are generally lower and some components (for example, the carrier of the $1700 \mathrm{~cm}-1 \mathrm{IR} \mathrm{C}=\mathrm{O}$ feature) are dramatically less abundant. This suggests that contamination associated with the operational environment of the spacecraft during flight was not a major source of sample contamination.

\subsubsection{Contamination from SRC Recovery and Curation Operations}

The accumulation of local soils (mud) from the recovery site was a major issue of concern for the Stardust Science Team prior to recovery of the SRC. Fortunately, integrity of the SRC during landing, the relative inability of the mud at the recovery site to stick to the SRC, and the fact that none of the bouncing impacts occurred at the locations of the two backshell vents greatly decreased the magnitude of this concern. Thus far, there is no indication that soils from the recovery site infiltrated the sample canister or in any way contaminated the returned samples. However, an unfortunate decision was made to place the recovered SRC into a polypropylene bag during the brief helicopter transport from the landing site to a hangar for preliminary deintegration operations. Subsequent detailed analysis revealed that the aerogel soaked up outgassed organics from this bag, providing an additional source 
of organic contamination to the comet coma grains (Hope Ishii, personal communication, 2007). One important lesson learned from the stardust mission is that recovery operations for the SRC significantly suffered from the lack of a hermetic seal for the samples, probably in many additional ways that will only become apparent in the future. Mission engineers should be pushed to provide truly hermetic seals for future returned samples. Contamination from curatorial operations has been carefully mitigated, although there is some evidence that the Stardust Interstellar aerogel has collected some contamination during handling in various labs during analyses (Bechtel et al. 2011).

\subsection{Hayabusa Mission}

The curation and contamination knowledge of the samples recovered from asteroid Itokawa by the Hayabusa mission (2004-2010) are very well described by Yada et al. (2014). In order to limit contamination to the recovered samples, the constituents of the Hayabusa sampler were limited to A6061 aluminum alloy coated with pure aluminum, stainless steel (304), Viton, aluminum oxide glass, and Teflon. Before launch, every part of the sample container was cleaned in 2-propanol using an ultrasonic cleaner, installed in an ISO Class 7 cleanroom. A contamination coupon made of aluminum oxide glass was installed inside the sample catcher to monitor contamination during the mission. In order to minimize ground contamination following Earth return, the sample container was designed to seal the samples, though the mission budget did not permit a hermetic seal. However, terrestrial atmosphere permeating through the double O-rings seal was estimated to be $<1 \mathrm{~Pa}$. During atmospheric entry, the sample container was designed to experience less than $80^{\circ} \mathrm{C}$ by using carbon fiber-reinforced plastic (CFRP) capsule ablators. The actual temperature from recovery on the Australian desert until introduction to the JAXA cleanroom was monitored with a temperature logger attached to a transportation box for the reentry capsule. The data of the logger showed that the sample container had been kept under $30{ }^{\circ} \mathrm{C}$. The JAXA cleanrooms are maintained under $26^{\circ} \mathrm{C}$. The magnetic condition of Hayabusa-returned samples should have been disturbed during a return trip to Earth due to the Hayabusa ion engine operation. Additional electric disturbance and shock from atmospheric entry, landing, and transportation, which might affect the samples, are still poorly understood.

\subsubsection{JAXA Hayabusa Lab}

The JAXA Hayabusa curation laboratory (hereafter "curation lab") consists of four cleanrooms of different clean levels: a planetary sample handling room (ISO Class 5 to 6), an electron microscope room (ISO Class 6), a sample preparation room (ISO Class 6), and a manufacturing and cleaning room (ISO Class 7). These have vertical air flow from the ceiling into a raised, perforated stainless steel floor. All filters used in fan filter units are polytetrafluoroethylene (PTFE), and an additional chemical filter absorbs acid gases such as halogen, sulfate, nitrate, elemental boron, and borate. Design of this lab made effective use of decades of knowledge from NASA's curation labs, and then significantly improved upon them.

Four special-use rooms exhaust their air independently to outside of the cleanrooms to protect the other cleanrooms from chemical and particle contamination. Additionally, there is a basement for equipment that cannot be set in the cleanrooms, such as roughing pumps for vacuum systems, a compressed air supply system, an ultra-pure water supply system, and nitrogen purifiers. 
The curation lab has two clean chambers for initial sample handling, Nos. 1 and 2. These are constructed mainly of 304 stainless steel, and their inside walls were electrochemically polished. They were baked in vacuum to at least $120{ }^{\circ} \mathrm{C}$ before and after the installation to reduce residual contamination. Both chambers are equipped with turbo molecular pumps (TMPs) and dry scroll pumps. Clean nitrogen supplied by a cyclic type nitrogen purifier and a flow type nitrogen purifier. The former is directly connected to each of the chambers to exclude $\mathrm{H}_{2} \mathrm{O}, \mathrm{O}_{2}$, and hydrocarbon from the circulating nitrogen. The chambers operate at positive pressure to exclude ambient air. Chamber 1, where the Hayabusa capsule was initially opened, can be operated at conditions of ultrahigh vacuum or purified nitrogen. Residual gas expanding into the chamber from the container was collected in bottles made of stainless steel. The lower part of the sample container was maintained in cabinet No. 1 under a vacuum. Chamber No. 1 was also equipped with Viton gloves through gate valves permitting the sample container and catcher to be manipulated with special tools.

After opening, the sample catcher was sent to chamber 2 (in nitrogen) for the extraction of captured Itokawa grains. Both chambers 1 and 2 contain ultraviolet (UV) neutralization lamps to compensate electrostatic charge, which should occur in the pure nitrogen condition. Also, an alpha-ray neutralizer containing a grain of ${ }^{210} \mathrm{Po}$ is employed for the same purpose - use of this neutralizer was pioneered in NASA's Stardust and Cosmic Dust Labs. The clean chambers were constructed using stainless steel (304 and 316), aluminum, and A6061 aluminum alloy, quartz glass, PTFE, and Viton. Gold and copper are used for materials in Itokawa grain sample holders, borosilicate glass for containers of less important items, and polyetheretherketone (PEEK) for electric connectors.

\subsubsection{Measurement of Gas in the Capture Cell upon Initial Opening}

Residual gas sampling bottles connected to clean chamber 1 were prepared to capture gas released from the container at the time of its initial opening (on June 23, 2010, 13 days after capsule recovery). As it expected that the container could contain some terrestrial atmosphere, $\mathrm{O}_{2}$ and ${ }^{40} \mathrm{Ar}$ could be used to identify any leaks into the "sealed" sample container.

Noble gases sampled in the gas bottles were analyzed at the University of Tokyo (Okazaki et al. 2011). Elemental ratios of the noble gases collected from the sample container were essentially identical to the terrestrial atmosphere. The inner pressure of the sample container was much higher than expected. Possible causes are a small leak of air through the double Viton O-rings seal, larger-than-expected permeability of the Viton O-rings, or a temporary leak of air accidentally happening during deintegration of the sample return capsule.

\subsubsection{Sample Removal from the Container}

On 24 June, 2010, the sample container was transferred to the transportation chamber from clean chamber 1 and the inner lid and the sample catcher were set into a catcher handling container. The inner lid, which was connected to the cover of sample catcher room A (there was also a room B), was removed, and its inner surface was observed and photographed. The catcher was placed into the catcher handling container, and these were transferred to clean chamber 2 . The inner surface of catcher room A was observed in detail by optical microscopy, and very few particles larger than a few hundred micrometers were observed inside room A.

Initial attempts to remove Itokawa grains from the aluminum sample catcher were unsuccessful. Without exception, every suspected grain proved to be a small piece of protruding Al metal. Next, a special PTFE spatula was used to sweep the interior surface of catcher 
room A. Observation of the spatula in the FESEM showed the presence of hundreds of rocky particles 1-30 $\mu \mathrm{m}$ in size, half of which proved to be Itokawa regolith grains (based on EDX spectra, Nakamura et al. 2011). Finally, the inverted sample catcher rooms A and B were tapped with a screw driver, causing a rain of Itokawa and Al grains to fall onto specially-prepared quartz glass disks. The particles were moved from the quartz disks to copper SEM mounts for examination by FESEM-EDS at low $\mathrm{kV}$ and no conductive coating. It was thought that particles analyzed by FESEM might be contaminated by vacuum pump hydrocarbons. However, Naraoka et al. (2012) showed that no measurable hydrocarbons could be detected on identically-treated witness surfaces by time of flight-secondary ion mass spectrometry.

Because of differences in the requirements for proposed analyses, sample containers used in the initial analyses of Itokawa grains varied. The samples analyzed for the mainstream of initial analyses, including synchrotron X-ray computed tomography and diffraction, FESEM and FE electron microprobe analysis, and a secondary ion mass spectrometry were embedded in epoxy resin and mounted onto glass fibers. They were transported within a stainless steel container filled with nitrogen. Samples for transmission electron microscopy were mounted in epoxy resin in a special nitrogen glovebox and transported within the same containers. In this case, they were processed without exposure to air. Samples for noble gas analyses were set in holes in a stainless steel base in a special flange of stainless steel, which had been baked beforehand to decrease contamination. This process was performed in nitrogen. Samples for organic analyses and instrumental neutron activation analysis were set in holes in a diamond plate with a diamond cover. Each investigation required special sample handling and encapsulation procedures.

\subsubsection{NASA’s Hayabusa Curation Laboratory}

Ultimately, $10 \%$ of the captured Itokawa grains will be transferred to NASA, and curated in the Hayabusa Curation Laboratory at JSC, although at the time of this writing less than 100 grains have been transferred to NASA's care. Sample containers for the NASA samples distribution consists of a pair of vacuum flanges of stainless steel as an outer container and a pair of synthetic quartz glass plates as a case to enclose the samples. All parts of the containers are separately cleaned by JAXA. The samples are placed into the quartz dimple slides using an electrostatically-controlled micromanipulator in clean chamber 2 at JAXA. The flanges are sealed with six screw bolts and oxygen-free copper gasket coated by gold. The sealing was also performed in the clean chamber, so the inside of the container was filled with atmospheric pressure nitrogen in the clean chamber. Only three materials are used for these sample containers: synthetic quartz, gold, and stainless steel. The NASA Hayabusa Cleanroom is a single room, containing at its core a stainless steel and glass cabinet for sample storage. No special sample handling is performed in this lab, as of now. Samples are merely stored, and allocated as needed.

\subsection{OSIRIS-REx}

Contamination knowledge for the OSIRIS-REx mission was covered in detail by Dworkin et al. (2018). This section will be a broad overview of the approaches and implementation of CK for OSIRIS-REx. Because curation scientists were involved in mission planning from the start, level 1, 2 and 3 mission requirements address aspects of $\mathrm{CK}$ and are integrated into the Contamination Control and Contamination Knowledge Plans. Because the mission is focused on amino acids and in general a carbonaceous asteroid, contamination efforts 
included attention to organics. An amino acid baseline of $180 \mathrm{ng} / \mathrm{cm}^{2}$ for OSIRIS-REx was based partly on analysis of Stardust foils, most of which was from a known contaminant called epsilon amino caproci acid (EACA), which is derived from hydrolyzation of nylon (Elsila et al. 2009). The planning for CC/CK involved identification of restricted materials and assessment of hydrazine contamination (from monoprop thrusters), ATLO cleanroom and payload faring monitoring, coupon and material archiving, flight system witness plates, and sample container air filter system. All of these activities and categories have led to a detailed understanding of potential contaminants for the collected sample.

\subsubsection{Materials Restrictions}

The OSIRIS-REx team was already aware of several specific and classes of compounds that would need to be avoided or restricted due to contamination concerns such as nylon and organic polymers (e.g., silicones, lubricants, adhesives). However, through open communication channels with engineers, additional materials or components were identified in advance, allowing ample time for identification of substitutes. For example, one process required diamond abrasives while another used a coating that included amorphous silica; both nanodiamonds and amorphous silica may also be present in primitive asteroid materials. The diamond-abraded surface was cleaned and verified diamond-free at JSC via FTIR, and the silica-containing material was removed. Galling (i.e., wear resulting from adhesion between sliding surfaces) is a frequent problem in spacecraft assembly and can be mitigated using various lubricants. This unavoidable use of lubricants is an example where material archiving can be helpful, and indeed the Braycote lubricant was archived for every use on the spacecraft during ATLO. Open communication between the subdiscipline engineers also led to the chemical investigation of products whose chemical makeups were unclear and/or proprietary. Analyses at GSFC and JSC allowed materials of concern to be tested in more detail, including couplants and adhesives, in several cases helping to identify replacement products (Dworkin et al. 2018). Detailed reports of materials testing were shared with the mission contamination knowledge scientists and placed on the internal science team website for review.

\subsubsection{Hydrazine}

OSIRIS-REx thruster propellant, hydrazine, is known to react with organics via a WolffKishner reduction (Dworkin et al. 2018). The mission team conducted tests of the reactivity of various organic compounds with anhydrous hydrazine, and decided that spacecraft thrusters should be canted away from the sampling site, which would result in $<180 \mathrm{ng} / \mathrm{cm}^{2}$ hydrazine to be deposited on TAGSAM surfaces. Even this hydrazine will rapidly evaporate from bare metal at sampling temperatures, but traces might be adsorbed by minerals or react with free carbonyls. In addition, drawing on experience from the Mars Phoenix lander mission and carrying out new calculations specific to OSIRIS-REx, mission engineers were able to estimate the amount of unreacted hydrazine in a thruster plume seeing the sample would be $<120 \mathrm{ng} / \mathrm{cm}^{2}$ for a single collection event. The only times when the spacecraft thrusters could deposit hydrazine onto the TAGSAM head are when the head is in the sampling configuration. This occurs during initial deployment and checkout, baseline samplemass measurements, the TAG rehearsals, and the TAG event(s). All these considerations led to a much better understanding of potential for hydrazine interaction with the samples, and alleviated concerns for contamination. 


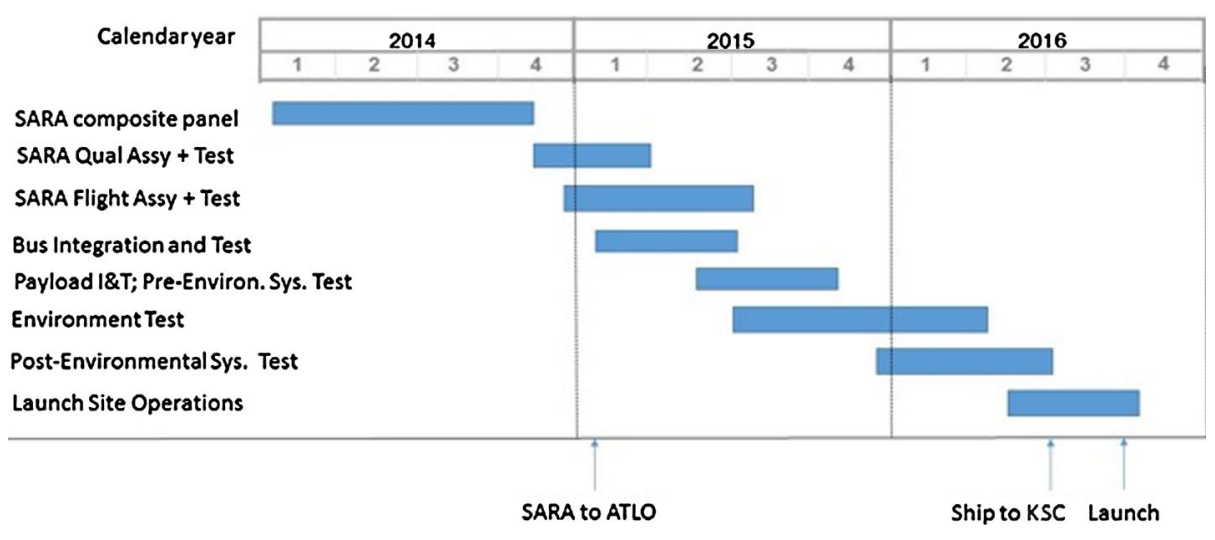

Fig. 13 Simplified schedule for SARA development, fabrication and assembly, and OSIRIS-REx Assembly, Testing and Launch Operations (ATLO)

\subsubsection{Materials and Coupon Archiving}

Archiving of materials identified to be of potential concern included those associated with the construction of the spacecraft, launch vehicle, SRC, TAGSAM (and associated hardware), science instruments, and materials used for packaging, containment, and processing of samples. During spacecraft assembly, the science and curation teams worked with the OSIRIS-REx mission engineers and ATLO personnel to archive materials from the spacecraft. Archiving began in February 2014, with work on the sample acquisition and retention assembly (SARA) composite panel in Denver, peaked near launch, and was completed by January 2017. Additionally, as the instruments were assembled and readied for integration, members of the instruments team packaged materials to send to JSC. As instruments and sub-assemblies of the spacecraft were tested and integrated, material coupons and items were sent to JSC through integration at KSC, with the last items having arrived at JSC in early 2017. A total of 406 items were received for the non-flight contamination knowledge collection. Data archived for each item also includes photos, its location on the spacecraft, physical description, the company that made the item along with its webpage or other contact information, the archiving location, archiver, and date. The materials fall into general categories including metals (stainless steel, aluminum, titanium alloys), epoxies, paints, polymers, lubricants, non-volatile-residue samples (NVR), sapphire, and various miscellaneous materials (a detailed list of items is found in Dworkin et al. 2018). The collection of CK materials, including witness plates, is archived and stored at NASA JSC in an ISO 7 cleanroom in dedicated stainless steel desiccator cabinets, with separately supplied dry nitrogen lines.

\subsubsection{Monitoring ATLO Cleanrooms: LM, KSC, and Payload Faring}

Witness plates were deployed to monitor cleanliness levels in the spacecraft assembly cleanrooms, high bay, environmental test facilities, spacecraft transport containers, Kennedy Space Center (KSC) Payload Hazardous Servicing Facility (PHSF), and the interior of the Atlas V launch vehicle fairing up until one day prior to launch of O-REx. All through the ATLO process (from March 2015 until late August 2016; Fig. 13) Si wafer and Al foil witness plates were deployed in these areas at LM and KSC to provide a record of particle counts and volatiles for current and future scientific studies. These plates were deployed in 
roughly monthly increments for 16 months with each unit containing $4 \mathrm{Si}$ wafers and $4 \mathrm{Al}$ foils, for a total of 128 individual witness plates (64 Si wafers and $64 \mathrm{Al}$ foils). One of each witness plate ( $\mathrm{Si}$ and $\mathrm{Al}$ ) was analyzed immediately, while the remaining three are archived at JSC with the materials archive (described in previous section) for future analysis. In addition to the witness plates, gas samples were collected in selected ATLO locations such as cleanrooms and testing facilities, with the goal of identifying any unexpected or problematic species, although no unexpected species were identified.

\subsubsection{Flight Witness Plates and Air Filter CK}

To witness the environment experienced by the sample collection system on the spacecraft, a series of witness plates were designed and implemented in three different areas in the sample canister-the top of the TAGSAM head, the TAGSAM wrist joint assembly, and the inside of the sample canister. The aluminum and sapphire witness plates are designed to be deployed on TAGSAM and in canister recording three different exposure timeframealways, pre-stow, and post-stow. These plates will be removed immediately upon return to JSC in the cleanroom and then stored in dedicated cabinets for contamination assessment studies. In addition to the witness plates, the sample canister has a two-way air filter in its lid which protects the sample from external contaminants, but the lid can also trap any volatile or particulate material from leaving the canister after TAGSAM is stowed. The filter performance was tested for moisture, particulate, and organic trapping efficiency during prelaunch activities and also drew heavily on similar filters used for the Stardust mission.

Additional contamination knowledge activities will continue during Phase E of the mission (Sept. 2016 to September 2023) with archiving of materials associated with the cleanroom construction at JSC and materials from the UTTR recovery site (might include soil, air, and other environmental background materials that could pose a contamination risk to OSIRIS-REx samples).

\subsection{Mars 2020 Rover Mission}

Although the Mars 2020 rover mission is not a sample return mission itself, it will be collecting and caching samples from the martian surface that could be picked up by a future mission, and hence Mars 2020 may represent the first mission in an overall Mars sample return campaign. Apart from in situ surface science, the goal of this first mission is to assemble a collection of rigorously documented and returnable cached samples. Contamination knowledge samples from Mars 2020 are in the process of being collected and curated to be part of the overall Mars sample collection if the cached samples are eventually returned.

While there are many aspects of the MSR contamination knowledge samples that will be similar to previous sample return missions, this sample return campaign has presented a number of unique opportunities and challenges. Perhaps the most notable is that due to the possibility of extinct or extant life on the surface of Mars, this sample return mission is designated as restricted by NASA's Planetary Protection Office. This designation requires more stringent organic contamination control requirements as well as the addition of biological contamination control and CK. Furthermore, this designation requires that the returned martian samples are curated in a containment facility in order to protect Earth from possible martian biohazards. The second notable difference is that the Mars 2020 mission would be part of a MSR campaign. Therefore, not only will the CK collection need to be coordinated between multiple missions, but significant point sources of possible contamination will remain on the martian surface. This mission architecture could make it more challenging to 
track a contaminant to its source. To minimize this knowledge gap, the CK samples collected will have to be more extensive than traditional sample return mission architectures. Finally, the Mars 2020 mission was not officially considered a sample return mission from its inception, so interaction with curation personnel and/or appropriate curation expertise was delayed and did not occur during the early stages of mission design, which has added an extra complication given the additional costs and time constraints of assembling a comprehensive CK collection.

\subsubsection{Contamination Knowledge Samples}

As with other sample return missions, an array of CK samples will be collected throughout ATLO. These samples will range from high fidelity flight reference materials to airfall witness samples. The flight and non-flight hardware and spacecraft components considered for $\mathrm{CK}$ are those items that are a potential contamination risk to the sample intimate hardware during launch, cruise, Mars entry/decent, landing, and rover surface operations. These "lineof-site" items are determined based on the Master Equipment List (MEL) and the Master Material List (MML). The MML will also be utilized to determine materials that have any potential to shed particulates and/or outgas molecular organics, directly or indirectly impacting the sample and caching subsystems. These samples can range from paint samples to flight spares or flight spare equivalents. Given the expanded scope of CK samples, an array of non-flight reference materials could help in tracing contamination. Some of these non-flight reference materials include: facility tools, equipment, and environmental components used during the fabrication, part processing, precision cleaning, and assembly that are considered a potential contamination risk. Witness items (e.g. plates and wipe samples) will be deployed within cleanrooms and on flow-benches during the assembly of the sample and caching subsystem. These witness items are duplicates of the witness items deployed and taken for CC and PP verification. All data collected during CC and PP verification will be tied to the respective $\mathrm{CK}$ sample, integrated into the $\mathrm{CK}$ database, and made available to the scientific community. Finally, unlike all other CK collections stored in nitrogen at ambient laboratory temperatures, the introduction of biological $\mathrm{CK}$ samples add the requirement to curate frozen samples $\left(<-80{ }^{\circ} \mathrm{C}\right)$.

\subsubsection{Innovation in Sample Storage}

Due to the stringent organic, inorganic, and biological CC and PP requirements, new ways to secure and store CK samples were developed for Mars 2020. Due to organic contamination concerns, all storage bags utilized for organic CK cannot be heat-sealed, so we developed customized bag clips. These two piece bag clips are constructed from 300 series stainless steel and Teflon. These clips will provide a strong seal that will ensure sample safety during shipment and long-term curation, and they will be used for future missions.

For the long term storage of CK samples, a few different storage containers needed to be upgraded or designed from scratch. Due to size constraints, this most high fidelity sample intimate hardware will be stored in a custom 316 stainless steel bolt-top canister with a Teflon seal, plumbed to accommodate an inert atmosphere to ensure sample safety in case of a breach in the primary containment. However, the bulk of the inorganic and organic reference and witness items will be stored within highly customized desiccators. As with the bolt-top containers, this highly customized desiccator is a modified version of the desiccators utilized for the other Astromaterials collections. However, due to stringent contamination control, the construction materials are highly limited. For example, unlike other desiccators 
that can utilize traditional flexible and highly compressible multi-use gasket material, the organic contamination requirements for Mars 2020 preclude their use. Therefore, a new door gasket design was required. Due to its low outgassing properties, a Teflon gasket was the preferred material. However, the trade-off for low outgassing is low flexibility and low compressibility, which means a whole new door gasket design was required. The new design leverages the slight compressibility of the Teflon gasket material while also utilizing it as a barrier material between a possible contaminant and the samples.

The collection of Mars $2020 \mathrm{CK}$ is an ongoing activity that will continue until the spacecraft is launched from Kennedy Space Center in July of 2020. Full details of the contamination knowledge collection and the laboratories that support these samples will be described in subsequent publications through joint efforts between the Mars 2020 science team and JSC curation personnel after a decision is made regarding the overall MSR campaign, which is expected sometime in 2020 .

\section{Preliminary Examination of Samples}

The preliminary examination (PE) of returned samples, be they collected from an extraterrestrial body and returned by spacecraft or recovered from a frozen lake or someone's back yard on Earth, is arguably the first step in their curation. The importance of this step cannot be overstated with respect to either the early identification of the sample type or the careful preservation of planetary materials for the future. Preliminary examination affects both of these, and many activities in between. Simply defined, preliminary examination is the process by which returned samples are documented and characterized to the point that the appropriate scientific research community is provided with enough information to select and request the samples for their individual, PI-led scientific studies. The results of preliminary investigations are typically presented in catalogs or online databases that are publicly available, and preliminary examination is considered to be a science-enabling activity.

\subsection{Steps Involved in Preliminary Examination}

The very first steps of preliminary examination of samples may take place before they are even collected. For all returned samples, the astronauts and/or instruments onboard the spacecraft (orbiter, lander, or rover) will gather as much data as possible about the surface of the body from where the samples are gathered. These data will likely include photographs, spectroscopic data, and other possible measurements given the available instruments/crew. For samples collected on the ground, such as meteorites, ideally, a photograph including a scale bar with an indication of compass orientation, a general description of the sample (e.g., percentage of fusion crust, possible rock type, notable physical characteristics), and a description of the site where the sample was found or any other noteworthy features. Care should be taken during these steps to minimize exposure to contamination sources, and any potential contamination should be documented in the collection notes; a list of recommended procedures and materials for collection of freshly-fallen meteorites is provided in Herd et al. (2016). Photographs of sample containment vessels may occur during and after collection. At the very least, the type of sample containment/transport vessel should be documented, to inform future sampling and curation documentation as well as subsequent scientific analysis. In all cases, the utmost care should be taken to ensure curation best practices are implemented to minimize forward contamination. 
Once samples are received in a curatorial facility, documentation of their current state should be made. These details may include information about the type of materials that the astromaterials samples were transported in, the state of those materials after landing (e.g., are seals intact? are they covered in dust?), and the documentation should include photographs. Any sampling of head gas in sample containment vessels that would be required should be completed and documented before sample containers are opened. Once the sample vessels are breached, information that may be of interest to research will be lost, along with the opportunity to ever gather it on those particular samples again. In addition, any tomographic scanning (e.g., X-ray or neutron) that is required to occur before samples are opened should be completed, and all processes involved in those analyses should be carefully documented.

Once samples are opened and, if applicable, removed from their sample containment vessels, the initial, curatorial steps involved in basic characterization of these materials take place. The main purpose of these efforts is to document exactly how the sample existed when it was opened. A documentation should be made as to the sample mass and its appearance at the time of opening. There should be a written description of the state of the material (intact rock, crumbled rock or sediments, powder, microscopic grains in a gel, etc.), its general attributes (color, grain size, physical appearance) and any notable features that are present (veins, fractures, fusion crust, metal content, etc.). Of utmost importance is photographic documentation (possibly with video, depending on the type of samples). Sample numbers should be assigned during this phase if they were not designated during collection. In short - the initial characterization of samples includes any process that can take place without making changes to the state of the samples other than opening their sample containment device, which is obviously unavoidable. Ideally, these steps should be made without touching the samples with anything other than curatorial tools comprised of materials determined to make contact with the samples without compromising their pristine nature. Determination of appropriate materials to use are based on several factors and typically represents a compromise between functionality and contamination risk, but foremost those materials must not compromise the ability of the samples to be used to answer the primary science requirements for a mission.

After basic characterization is completed, the curatorial phase of preliminary examination of the samples begins. Preliminary examination is distinct from science activities, and its goal is to produce a sample catalog with a level of detail about each sample that is sufficient for members of the scientific community to make informed requests of materials to conduct their PI-led scientific investigations. Preliminary examination of materials can occur for each representative portion of a sample, if needed to produce a meaningful and informative sample catalog. For Preliminary examinations on samples that also have a mission science team, preliminary examination can happen in parallel with science activities, but the goals of these two activities remains distinct. The methods and analytical techniques used during preliminary examination of a sample will be tailored to the primary science requirements for that sample. Furthermore, these processes will be determined based on sample size, sample form, sample vessel, and the need to prevent either forward or backward contamination. Large samples such as meteorites need to be touched with tools/gloves to be weighed, photographed, and described, and they need to be broken with tools to provide material for their classification. The next steps of preliminary examination of meteorites require the classification chip to be processed even further. As part of the U.S. Antarctic Meteorite Program (AMP) the smaller meteorite chip is weighed, placed into a sample container, and sent from NASA JSC to the Smithsonian National Museum of Natural History for further visual examination via binocular microscope, chipping, insertion into a sample holder grid, and polishing for energy dispersive spectroscopic (EDS) analyses, and/or made into a pet- 
rographic thin or thick section (both of which involve exposure to epoxy and polishing grit). These samples are coated with carbon and analyzed with a scanning electron microscope with EDS and/or an electron microprobe to determine their mineral compositions (namely olivine, pyroxene, and in the case of iron meteorites, FeNi metal). Other collections contain much smaller specimens and require much more careful micromanipulation of materials as discussed previously in the small particle handling section.

Preliminary examination of gas and volatile-rich samples present unique challenges to the curatorial preliminary examination process. In the gas phase, samples cannot be photodocumented or weighed. Condensed volatiles can be weighed, but only as a supplement to total or partial-pressure measurements of the quantity of sample in the gas phase. Whether the sample is condensed or not, total and partial pressures of major species (e.g. $\mathrm{H}_{2} \mathrm{O}, \mathrm{CO}_{2}$, etc., depending on the sample origin) should be monitored using techniques that consume little to no sample. Spectroscopy-based techniques (e.g., FTIR, Raman, cavity ring-down spectroscopy) provide possible non-destructive means by which an initial characterization of the compounds in a sample can be ascertained; however, care must be taken to ensure that the techniques (and wavelengths) used do not pose a risk of altering the sample composition or isotopic distribution. High-sensitivity gas chromatography-mass spectrometry (GC-MS) or other high-sensitivity gas analysis techniques can be used to supplement spectroscopy using small quantities $(<1 \mathrm{~g})$ of sample.

The concept of a "representative sample" for allocation purposes requires further development for gas and volatile-rich samples. Depending on the temperature at which preliminary examination takes place, some species may be condensed while others remain in the gas phase. Additionally, thorough mixing of a gas-phase sample may be impossible to guarantee, especially while the sample remains sealed in the flight sample container. Therefore, when producing aliquots of gas samples for distribution to the scientific community, homogeneity between aliquots should not be expected. One possible solution is to separate gas-phase compounds by their condensation temperatures, freezing out compounds in a sequence that allows them to be separated by composition. This would limit (and possibly prohibit) bulk compositional analyses, but it would separate the sample into known compositions from which aliquots could be obtained. Regardless of how representative samples are defined, significant development is still required to address this capability gap for future volatile-rich sample return missions. An alternative to conducting preliminary examination of gas samples is to immediately conduct analysis of the gases for scientific purposes prior to preliminary examination. This option would be desirable in any instance where the primary science goals for a sample could be compromised through any preliminary examination processes.

Once the data are collected during preliminary examination, they are compiled and released to the scientific community in various online/digital formats or sample catalogs in order to allow researchers to request them for study. For U.S. meteorites, this is the Antarctic Meteorite Newsletter published in February and September each year (for example, https://curator.jsc.nasa.gov/antmet/amn/amn.cfm\#n412). For other collections, different mechanisms of reporting of available materials are put in place. All NASA curated samples are detailed in the ARES website (https://curator.jsc.nasa.gov/), and new samples announced biennially in the Astormaterials Newsletter (https://curator.jsc.nasa.gov/newsletter/\#n0101). And meteorites from around the world are detailed in the Meteoritical Bulletin (https://www. lpi.usra.edu/meteor/metbull.php).

Preliminary examination does not end with the process of reporting data in a newsletter. The curation process regularly involves the subsampling of materials. Each time this occurs, 
the subsampling process is ideally well documented with photographs, diagrams of subsampling, the weights of both removed and remaining materials, and descriptions of those materials if noteworthy. The process of subsampling larger samples involves exposing new material to the sample surface and to the curatorial environment. These newly exposed materials may require description, and in extreme cases, if they reveal something extraordinary, may necessitate announcement to the scientific community of new sample opportunities. Preliminary examination, unlike initial characterization, will go on as long as there is sample remaining in our collections, and hence sample catalogs are living documents.

\subsection{Who Does PE?}

Who should be involved in the preliminary examination of samples depends on the mechanism by which the samples were recovered. For spacecraft missions to planetary bodies within our Solar System, there are numerous stakeholders. These stakeholders include the mission science team that orchestrated and successfully executed the sample return mission, the scientific community at large that will also want to study the returned samples, and future generations of scientists that will want to study these samples with technology that has not yet been invented. It is the responsibility of a collection curator to defend all of these stakeholders and to find the right balance between sample consumption and sample conservation that maximizes science returns on the samples over multi-decade timescales. Given the important role of a collection curator for the safety and long-term viability of returned samples, it only makes sense for astromaterials returned from spacecraft missions to be received, opened, processed, and characterized within a sample curation facility by specially trained personnel under the management of a curatorial authority. That said, the people involved in the preliminary examination of returned materials will not be limited to curation personnel. The people involved in the preliminary examination should include some combination of curatorial processors trained to process and document miniscule samples, collection curators, and members of the sample science team. There may be a healthy tension that develops between the curator and members of a sample science team (i.e., conservation vs. consumption, respectively) because the science team has scientific mission requirements to achieve within a fixed period of time, and the curator must think beyond that time frame to the long-term viability and availability of the samples. To minimize such tensions, it is important to have policies in place prior to the samples being returned that outline how much sample can be consumed by the mission science team to achieve the primary science goals.

With less controlled sample collection, such as meteorite falls recovered anywhere in the world, the very first steps of preliminary examination (i.e., basic characterization) may take place in the field by trained meteorite hunting/recovery programs such as those run by the US (ANSMET), Japan, Belgium, China, South Korea, and the UK. The ANSMET program, for example, documents the field location of each meteorite recovered with a GPS position, a photograph next to a field number, the percentage of fusion crust visible on the sample, an educated guess at the meteorite type, a general description of any other notable features (i.e., it was found in liquid water, it was found half buried in ice, it was broken in half), and anything else that may require comment (i.e., it was accidentally touched with a glove, a bare hand, a snowmobile) and could possibly affect future analyses. Once the field documentation is completed and the samples are sent to a curation facility, preliminary examination largely occurs by curation personnel, and the samples are made available for request without first being analyzed by a mission science team. 


\subsection{Where do We Draw the Line Between Preliminary Examination for Curation and Science?}

Basic characterization and preliminary examination are, as stated above, the processes by which returned samples are initially documented and sufficiently characterized to provide the appropriate scientific research community with enough information to select and request them for scientific study. A long standing discussion is carried on by curators worldwide as to what constitutes "too much characterization" and where the line is drawn between performing that characterization and conducting research that should be PI-led. The line is particularly difficult to delineate for small particle collections where the entire particle may be $<10 \mu \mathrm{m}$ and almost any observation with an electron beam or laser could alter the sample (e.g., interplanetary dust particles in NASA's Cosmic Dust collection). However, there are also some examples of disagreement among curators as to which instrumentation and analyses are appropriate for preliminary examination on large samples where subsampling does not negatively impact the availability of the material (e.g., oxygen isotopic measurements, $\mathrm{X}$-ray computed tomography, etc.). Nonetheless, the line between characterization and science is going to be different for each sample type, and that line should be optimally placed such that wasted sample consumption resulting from insufficient information about the samples in a catalog (i.e., the consumed sample did not have the phase of interest) is minimized whilst serendipitous discoveries within the samples during scientific investigations can still occur.

One of the questions meteorite curators commonly ask, for example, is whether or not oxygen isotopic compositions should be determined for meteorite samples returned from Antarctica or elsewhere. These measurements are very much in the realm of PI-led research, but with meteorites coming from a variety of different Solar System bodies, providing the $\Delta{ }^{17} \mathrm{O}$ composition gives the curator and requesting scientist the background information needed to identify potentially unique samples or those thought to be from Mars or other potentially unknown sources. Generally, isotope labs in the U.S. have provided the U.S. Antarctic Meteorite Program with these data as needed, but O-isotope analysis should only be used as a characterization tool when other, less destructive, methods cannot be used to uniquely classify the material. Another example is the use of X-ray computed tomography (XCT) as a characterization tool. Having three-dimensional context of samples provides curation personnel with invaluable information about the contents of samples, particularly highly heterogeneous samples like regolith breccias. This information can be used to identify clasts that are not exposed at the surface, and it can be used to make informed decisions about cuts or sample splits during sample processing. However, XCT exposes samples to radiation doses that can have lasting effects on the samples. Thermoluminescence in particular is negatively impacted by XCT analysis of samples (Sears et al. 2016, 2018), and studies are underway to better characterize its effect on organic compounds in samples (Hanna and Ketcham 2017; Friedrich et al. 2019).

Most curated astromaterials samples today are room-temperature solids; however, as we advance to collecting materials that require cryogenic storage or gas-phase samples, further questions arise as to how to conduct a preliminary examination, especially if the "shelflife" of the samples prohibit waiting for the technology of tomorrow to analyze the samples. An alternative approach to a preliminary examination for materials that may have a short shelf-life is to organize a large scale consortium study where all stakeholders in the scientific community are given the opportunity to join, either through an "all are welcome" approach like Stardust or competitively through a proposal process if there is a need to limit the number of investigators. Conservation for the sake of conservation is not a meaningful 
philosophical approach to astromaterials curation. It is important that the goals of maximizing science returns on samples over their viable lifetimes are an integral part of a long-term sample conservation plan.

\section{Looking Forward}

Advanced curation is a critical function to the success of sample return missions and Earthbased sample acquisition and plays an integral part in enabling the high precision measurements that are often done on astromaterials samples. Looking forward, advanced curation must prepare for sample return missions from any celestial body within the solar system, including planets, moons, asteroids, and/or comets. The direction and scope of advanced curation research is driven by (1) existing strategic knowledge gaps identified through lessons learned from previous sample return missions and Earth-based programs that collect astromaterials; (2) the emerging needs of the scientific community that study astromaterials samples; and (3) the selection of new targets for sample return missions and the associated curation and sample handling requirements of those missions (e.g., Beaty et al. 2019; Haltigin et al. 2018; McLennan et al. 2011; Vander Kaaden et al. 2019). The primary result of advanced curation is to both reduce and quantify contamination to astromaterials and preserve the scientific integrity of all samples from mission inception and through ATLO, sample collection, curation/preliminary examination on Earth, curation/storage, and secure delivery of the samples to Earth-based laboratories for in-depth scientific analysis. Advanced curation is an interdisciplinary field of research and development and also serves as an important science-enabling activity. The collective lessons learned from previous spacecraft missions and the results of advanced curation research will work in tandem to feed forward into better spacecraft designs and enable more stringent requirements for future sample return missions.

Acknowledgements This work was supported, in part, by NASA's Science Mission Directorate. We are grateful for the editorial handling of this manuscript by Sara Russell, and this work was improved on the basis of input from the editor as well as two anonymous reviewers. This work is dedicated to the women and men that have worked tirelessly to make sample return missions possible, to the countless astromaterials curation personnel that have processed and cared for astromaterials collections, and to the members of the scientific community that study these carefully curated astromaterials.

Publisher's Note Springer Nature remains neutral with regard to jurisdictional claims in published maps and institutional affiliations.

Open Access This article is distributed under the terms of the Creative Commons Attribution 4.0 International License (http://creativecommons.org/licenses/by/4.0/), which permits unrestricted use, distribution, and reproduction in any medium, provided you give appropriate credit to the original author(s) and the source, provide a link to the Creative Commons license, and indicate if changes were made.

\section{References}

P.I. Abell, C.H. Draffan, G. Eglinton, J.M. Hayes, J.R. Maxwell, C.T. Pillinger, Organic analysis of the returned Apollo 11 lunar sample, in Apollo 11 Lunar Science Conference, Proc., vol. 2 (Pergamon, New York, 1970), pp. 1757-1773

C.M.O.D. Alexander, G.D. Cody, Y. Kebukawa, R. Bowden, M.L. Fogel, A.L.D. Kilcoyne, L.R. Nittler, C.D.K. Herd, Elemental, isotopic, and structural changes in Tagish Lake insoluble organic matter produced by parent body processes. Meteorit. Planet. Sci. 49, 503-525 (2014) 
C.C. Allen, F.G. Albert, J. Combie, A. Banin, Y. Yablekovitch, I. Kan, R.J. Bodnar, V.E. Hamilton, B.L. Jolliff, K. Kuebler, A. Wang, D.J. Lindstrom, P.A. Morris, R.V. Morris, R.W. Murray, L.E. Nyquist, P.D. Simpson, A. Steele, S.J. Symes, Effects of sterilizing doses of gamma radiation on Mars analog rocks and minerals. J. Geophys. Res., Planets 104, 27043-27066 (1999)

C. Allen, J. Allton, G.E. Lofgren, K. Righter, M. Zolensky, Curating NASA's extraterrestrial samples-Past, present, and future. Chem. Erde 71, 1-20 (2011)

J.H. Allton, J.D. Hittle, E.T. Mickelson, E.K. Stansbery, Cleaning Genesis Sample Return Canister for Flight: Lessons for Planetary Sample Return (NASA Johnson Space Center, Houston, 2016), p. 41

T.J. Anchordoquy, M.C. Molina, Preservation of DNA. Cell Preserv. Technol. 5, 180-188 (2007)

L.D. Andrade, R. Awasthi, K. Dua, T.D.A. Pinto, Matrix-assisted laser desorption ionization-time of flight mass spectrometry for identification of bacteria isolated from pharmaceutical cleanrooms. Interv. Med. Appl. Sci. 10, 45-53 (2018)

J.O. Annexstad, W.A. Cassidy, Collecting and processing Victoria Land meteorites, in Memoirs of National Institute of Polar Research, Special Issue (1980), pp. 14-20

L. Aronowitz, C. Baulknight, J. Berkowitz-Mattuck, A. Buchler, P. Glaser, F. Koch, T. Luzzi, N. Milford, S. Penn, F. Pomilla, Investigation of lunar surface chemical contamination by LEM descent engine and associated equipment Final report. Grumman Aircraft Engineering Corp.; Research Dept.; Bethpage, NY, United States (1966a) p. 219

L. Aronowitz, C. Baulknight, A. Buchler, F. Koch, T. Luzzi, S. Penn, A. Wechsler, D. Weiss, Investigation of Lunar Surface Chemical Contamination by LEM Descent Engine and Associated Equipment. Grumman Aircraft Engineering Corp.; Research Dept.; Bethpage, NY, United States (1966b), p. 55

M. Bashir, M. Ahmed, T. Weinmaier, D. Ciobanu, N. Ivanova, T.R. Pieber, P.A. Vaishampayan, Functional metagenomics of spacecraft assembly cleanrooms: Presence of virulence factors associated with human pathogens. Front. Microbiol. 7, 12 (2016)

D. Beaty, C. Allen, D. Bass, K. Buxbaum, J. Cambell, D. Lindstrom, S. Miller, D. Papanastassiou, Planning considerations for a Mars sample receiving facility: Summary and interpretation of three design studies. Astrobiology 9 (2009). https://doi.org/10.1089/ast.2009.0339

D.W. Beaty, M.M. Grady, H.Y. McSween, E. Sefton-Nash, B.L. Carrier, F. Altieri, Y. Amelin, E. Ammannito, M. Anand, L.G. Benning, J.L. Bishop, L.E. Borg, D. Boucher, J.R. Brucato, H. Busemann, K.A. Campbell, A.D. Czaja, V. Debaille, D.J. Des Marais, M. Dixon, B.L. Ehlmann, J.D. Farmer, D.C. FernandezRemolar, J. Filiberto, J. Fogarty, D.P. Glavin, Y.S. Goreva, L.J. Hallis, A.D. Harrington, E.M. Hausrath, C.D.K. Herd, B. Horgan, M. Humayun, T. Kleine, J. Kleinhenz, R. Mackelprang, N. Mangold, L.E. Mayhew, J.T. McCoy, F.M. McCubbin, S.M. McLennan, D.E. Moser, F. Moynier, J.F. Mustard, P.B. Niles, G.G. Ori, F. Raulin, P. Rettberg, M.A. Rucker, N. Schmitz, S.P. Schwenzer, M.A. Sephton, R. Shaheen, Z.D. Sharp, D.L. Shuster, S. Siljeström, C.L. Smith, J.A. Spry, A. Steele, T.D. Swindle, I.L. ten Kate, N.J. Tosca, T. Usui, M.J. Van Kranendonk, M. Wadhwa, B.P. Weiss, S.C. Werner, F. Westall, R.M. Wheeler, J. Zipfel, M.P. Zorzano, The potential science and engineering value of samples delivered to Earth by Mars sample return. Meteorit. Planet. Sci. 54, S3-S152 (2019)

H.A. Bechtel, C. Allen, S. Bajt, J. Borg, F. Brenker, J. Bridges, D.E. Brownlee, M. Burchell, M. Burghammer, A.L. Butterworth, P. Cloetens, A.M. Davis, C. Floss, G.J. Flynn, D. Frank, Z. Gainsforth, E. Grun, P.R. Heck, J.K. Hillier, P. Hoppe, L. Howard, G.R. Huss, J. Huth, A. Kearsley, A.J. King, B. Lai, J. Leitner, L. Lemelle, H. Leroux, L.R. Nittler, R.C. Ogliore, F. Postberg, M.C. Price, S.A. Sandford, J.A. Sans Tresseras, S. Schmitz, T. Schoonjans, G. Silversmit, A. Simionovici, R. Srama, F.J. Stadermann, T. Stephan, J. Stodolna, R.M. Stroud, S.R. Sutton, R. Toucoulou, M. Trieloff, P. Tsou, A. Tsuchiyama, T. Tyliczszak, B. Vekemans, L. Vincze, A.J. Westphal, M.E. Zolensky et al., FTIR analysis of aerogel keystones from the stardust interstellar dust collector: Assessment of terrestrial organic contamination and X-ray microprobe beam damage, in 42nd Lunar and Planetary Science Conference, Abstract \#1971 (2011)

J.N. Benardini, K. Venkateswaran, Application of the ATP assay to rapidly assess cleanliness of spacecraft surfaces: A path to set a standard for future missions. AMB Express 6, 8 (2016)

A. Bieler, K. Altwegg, H. Balsiger, A. Bar-Nun, J.J. Berthelier, P. Bochsler, C. Briois, U. Calmonte, M. Combi, J. De Keyser, E.F. van Dishoeck, B. Fiethe, S.A. Fuselier, S. Gasc, T.I. Gombosi, K.C. Hansen, M. Hässig, A. Jäckel, E. Kopp, A. Korth, L. Le Roy, U. Mall, R. Maggiolo, B. Marty, O. Mousis, T. Owen, H. Rème, M. Rubin, T. Sémon, C.Y. Tzou, J.H. Waite, C. Walsh, P. Wurz, Abundant molecular oxygen in the coma of comet 67P/Churyumov-Gerasimenko. Nature 526, 678 (2015)

P.A. Bland, The Desert Fireball Network. Astron. Geophys. 45, 5.20-5.23 (2004)

P.A. Bland, P. Spurny, A.W.R. Bevan, K.T. Howard, M.C. Towner, G.K. Benedix, R.C. Greenwood, L. Shrbeny, I.A. Franchi, G. Deacon, J. Borovicka, Z. Ceplecha, D. Vaughan, R.M. Hough, The Australian Desert Fireball Network: A new era for planetary science. Aust. J. Earth Sci. 59, 177-187 (2012)

A.I. Blinova, C.D.K. Herd, M.J.M. Duke, Testing variations within the Tagish Lake meteorite-II: Wholerock geochemistry of pristine samples. Meteorit. Planet. Sci. 49, 1100-1118 (2014) 
J. Borovička, Z. Charvát, Meteosat observation of the atmospheric entry of 2008 TC over Sudan and the associated dust cloud. Astron. Astrophys. 507, 1015-1022 (2009)

F. Brandstätter, History of the meteorite collection of the Natural History Museum of Vienna. Geol. Soc. (Lond.) Spec. Publ. 256, 123-133 (2006)

C.E. Bresky, M. Fries, The Aquarius project: The first student-driven attempt to retrieve meteorites from underwater, in 49th Lunar and Planetary Science Conference, Abstract \#3004 (2018)

D. Brownlee, P. Tsou, J. Aleon, C.M.O. Alexander, T. Araki, S. Bajt, G.A. Baratta, R. Bastien, P. Bland, P. Bleuet, J. Borg, J.P. Bradley, A. Brearley, F. Brenker, S. Brennan, J.C. Bridges, N.D. Browning, J.R. Brucato, E. Bullock, M.J. Burchell, H. Busemann, A. Butterworth, M. Chaussidon, A. Cheuvront, M.F. Chi, M.J. Cintala, B.C. Clark, S.J. Clemett, G. Cody, L. Colangeli, G. Cooper, P. Cordier, C. Daghlian, Z.R. Dai, L. D’Hendecourt, Z. Djouadi, G. Dominguez, T. Duxbury, J.P. Dworkin, D.S. Ebel, T.E. Economou, S. Fakra, S.A.J. Fairey, S. Fallon, G. Ferrini, T. Ferroir, H. Fleckenstein, C. Floss, G. Flynn, I.A. Franchi, M. Fries, Z. Gainsforth, J.P. Gallien, M. Genge, M.K. Gilles, P. Gillet, J. Gilmour, D.P. Glavin, M. Gounelle, M.M. Grady, G.A. Graham, P.G. Grant, S.F. Green, F. Grossemy, L. Grossman, J.N. Grossman, Y. Guan, K. Hagiya, R. Harvey, P. Heck, G.F. Herzog, P. Hoppe, F. Horz, J. Huth, I.D. Hutcheon, K. Ignatyev, H. Ishii, M. Ito, D. Jacob, C. Jacobsen, S. Jacobsen, S. Jones, D. Joswiak, A. Jurewicz, A.T. Kearsley, L.P. Keller, H. Khodja, A.L.D. Kilcoyne, J. Kissel, A. Krot, F. Langenhorst, A. Lanzirotti, L. Le, L.A. Leshin, J. Leitner, L. Lemelle, H. Leroux, M.C. Liu, K. Luening, I. Lyon, G. MacPherson, M.A. Marcus, K. Marhas, B. Marty, G. Matrajt, K. McKeegan, A. Meibom, V. Mennella, K. Messenger, S. Messenger, T. Mikouchi, S. Mostefaoui, T. Nakamura, T. Nakano, M. Newville, L.R. Nittler, I. Ohnishi, K. Ohsumi, K. Okudaira, D.A. Papanastassiou, R. Palma, M.E. Palumbo, R.O. Pepin, D. Perkins, M. Perronnet, P. Pianetta, W. Rao, F.J.M. Rietmeijer, F. Robert, D. Rost, A. Rotundi, R. Ryan, S.A. Sandford, C.S. Schwandt, T.H. See, D. Schlutter, J. Sheffield-Parker, A. Simionovici, S. Simon, I. Sitnitsky, C.J. Snead, M.K. Spencer, F.J. Stadermann, A. Steele, T. Stephan, R. Stroud, J. Susini, S.R. Sutton, Y. Suzuki, M. Taheri, S. Taylor, N. Teslich, K. Tomeoka, N. Tomioka, A. Toppani, J.M. Trigo-Rodriguez, D. Troadec, A. Tsuchiyama, A.J. Tuzzolino, T. Tyliszczak, K. Uesugi, M. Velbel, J. Vellenga, E. Vicenzi, L. Vincze, J. Warren, I. Weber, M. Weisberg, A.J. Westphal, S. Wirick, D. Wooden, B. Wopenka, P. Wozniakiewicz, I. Wright, H. Yabuta, H. Yano, E.D. Young, R.N. Zare, T. Zega, K. Ziegler, L. Zimmerman, E. Zinner, M. Zolensky, Comet 81P/Wild 2 under a microscope. Science 314, 1711-1716 (2006)

A.L. Burlingame, M. Calvin, J. Han, W. Henderson, W. Reed, B.R. Simoneit, Study of carbon compounds in Apollo 11 lunar samples, in Apollo 11 Lunar Science Conference, Proc., vol. 2 (Pergamon, New York, 1970), pp. 1779-1791

A.L. Burlingame, P.T. Holland, W.H. McFadden, B.R. Simoneit, J.T. Wilder, P.C. Wszolek UCB Space Sciences Laboratory Organic Cleanroom and Lunar Material Transfer Facilities. Space Sciences Laboratory Report, University of California, Berkeley (1971)

D.S. Burnett, The Genesis solar wind sample return mission: Past, present, and future. Meteorit. Planet. Sci. 48, 2351-2370 (2013)

D.S. Burnett, B.L. Barraclough, R. Bennett, M. Neugebauer, L.P. Oldham, C.N. Sasaki, D. Sevilla, N. Smith, E. Stansbery, D. Sweetnam, R.C. Wiens, The Genesis Discovery mission: Return of solar matter to Earth. Space Sci. Rev. 105, 509-534 (2003)

A.S. Burton, D.P. Glavin, J.E. Elsila, J.P. Dworkin, P. Jenniskens, Q.Z. Yin, The amino acid composition of the Sutter's Mill CM2 carbonaceous chondrite. Meteorit. Planet. Sci. 49, 2074-2086 (2014)

CABRI, Laboratory procedures for microorganisms: Survey of examples of detailed protocols for the different types of preservation methods, in Common Access to Biotechnological Resources and Information Consortium (1998) http://www.cabri.org/guidelines/micro-organisms/M300Ap500.html. Accessed April 24, 2019

M.J. Calaway, Lunar processing cabinet 2.0: Retrofitting gloveboxes into the 21st century, in Lunar and Planetary Science Conference XLVI, March 16-20, The Woodlands, TX, Abstract \#1492 (2015)

M.J. Calaway, C.C. Allen, Cryogenic curation: Isolated technology and mission operational requirements for sample return, in 76th Annual Meteoritical Society Meeting, Edmonton Alberta, Canada (2013)

M.J. Calaway, D.S. Burnett, M.C. Rodriguez, S. Sestak, J.H. Allton, E.K. Stansbery, Decontamination of genesis array materials by UV ozone cleaning, in Lunar and Planetary Science Conference XXXVIII, Houston, TX, Abstract \#1627 (2007)

M.J. Calaway, M.C. Rodriguez, J.H. Allton, E.K. Stansbery, Decontaminating solar wind samples with the genesis ultra-pure water megasonic cleaner, in Lunar and Planetary Science Conference XL, Woodlands, TX, Abstract \#1183 (2009)

M.J. Calaway, C.C. Allen, J.H. Allton, Organic Contamination Baseline Study in NASA Johnson Space Center Astromaterials Curation Laboratories, NASA TP-2014-217393, Lyndon B. Johnson Space Center, Houston (2014), p. 108 
M.J. Calaway, J.H. Allton, R.A. Zeigler, F.M. McCubbin, 50th anniversary of the world's first extraterrestrial sample receiving laboratory: The Apollo program's lunar receiving laboratory, in 48th Lunar and Planetary Science Conference, Abstract \#1224 (2017)

Z. Ceplecha, J. Borovicka, W.G. Elford, D.O. Revelle, R.L. Hawkes, V. Porubcan, M. Simek, Meteor phenomena and bodies. Space Sci. Rev. 84, 327-471 (1998)

B.C. Clark, Temperature-time issues in bioburden control for planetary protection. Adv. Space Res. 34, 23142319 (2004)

A. Colaprete, P. Schultz, J. Heldmann, D. Wooden, M. Shirley, K. Ennico, B. Hermalyn, W. Marshall, A. Ricco, R.C. Elphic, D. Goldstein, D. Summy, G.D. Bart, E. Asphaug, D. Korycansky, D. Landis, L. Sollitt, Detection of water in the LCROSS ejecta plume. Science 330, 463-468 (2010)

F. Colas, B. Zanda, J. Vaubaillon, S. Bouley, C. Marmo, Y. Audureau, M.K. Kwon, J.-L. Rault, S. Caminade, P. Vernazza, French fireball network FRIPON, in Proceedings of the International Meteor Conference, Mistelbach, Austria (2015), pp. 27-30

W. Cooke, D. Moser, The status of the NASA all sky fireball network, NASA report, in Proceedings of the International Meteor Conference, 30th IMC, Sibiu, Romania (2011)

M. Cooper, M.T. La Duc, A. Probst, P. Vaishampayan, C. Stam, J.N. Benardini, Y.M. Piceno, G.L. Andersen, K. Venkateswaran, Comparison of innovative molecular approaches and standard spore assays for assessment of surface cleanliness. Appl. Environ. Microbiol. 77, 5438-5444 (2011)

COSPAR, COSPAR planetary protection policy (20 October 2002, as amended to 24 March 2011), in COSPAR/IAU Workshop on Planetary Protection, COSPAR (2011)

L.A. Dauphin, B.D. Moser, M.D. Bowen, Evaluation of five commercial nucleic acid extraction kits for their ability to inactivate Bacillus anthracis spores and comparison of DNA yields from spores and spiked environmental samples. J. Microbiol. Methods 76, 30-37 (2009)

B.H. Day, P.A. Bland, R. Sayers, Fireballs in the sky: Citizen science with the desert fireball network, in 49th Lunar and Planetary Science Conference, Abstract \#2229 (2018a)

J.M.D. Day, J. Maria-Benavides, F.M. McCubbin, R.A. Zeigler, The potential for metal contamination during Apollo lunar sample curation. Meteorit. Planet. Sci. 53, 1283-1291 (2018b)

S.F. Dermott, J.C. Liou, Detection of asteroidal dust particles from known families in near-Earth orbits, in AIP Conference Proceedings, vol. 3, no. 1 (AIP, New York, 1994)

J.P. Dworkin, L.A. Adelman, T. Ajluni, A.V. Andronikov, J.C. Aponte, A.E. Bartels, E. Beshore, E.B. Bierhaus, J.R. Brucato, B.H. Bryan, A.S. Burton, M.P. Callahan, S.L. Castro-Wallace, B.C. Clark, S.J. Clemett, H.C. Connolly, W.E. Cutlip, S.M. Daly, V.E. Elliott, J.E. Elsila, H.L. Enos, D.F. Everett, I.A. Franchi, D.P. Glavin, H.V. Graham, J.E. Hendershot, J.W. Harris, S.L. Hill, A.R. Hildebrand, G.O. Jayne, R.W. Jenkens, K.S. Johnson, J.S. Kirsch, D.S. Lauretta, A.S. Lewis, J.J. Loiacono, C.C. Lorentson, J.R. Marshall, M.G. Martin, L.L. Matthias, H.L. McLain, S.R. Messenger, R.G. Mink, J.L. Moore, K. Nakamura-Messenger, J.A. Nuth, C.V. Owens, C.L. Parish, B.D. Perkins, M.S. Pryzby, C.A. Reigle, K. Righter, B. Rizk, J.F. Russell, S.A. Sandford, J.P. Schepis, J. Songer, M.F. Sovinski, S.E. Stahl, K. Thomas-Keprta, J.M. Vellinga, M.S. Walker, OSIRIS-REx contamination control strategy and implementation. Space Sci. Rev. 214, 53 (2018)

ECSS, Space Product Assurance: Microbial Examination of Flight Hardware and Cleanrooms. European Cooperation for Space Standardization, ECSS-Q-ST-70-55C, Noordwijk, The Netherlands (2008)

W.N. Edwards, D.W. Eaton, P.G. Brown, Seismic observations of meteors: Coupling theory and observations. Rev. Geophys. 46, 21 (2008)

J.E. Elsila, D.P. Glavin, J.P. Dworkin, Cometary glycine detected in samples returned by Stardust. Meteorit. Planet. Sci. 44, 1323-1330 (2009)

D.A. Flory, B.R. Simoneit, Terrestrial contamination in Apollo lunar samples. Space Life Sci. 3, 457-468 (1972)

G.J. Flynn, L.P. Keller, C. Jacobsen, S. Wirick, An assessment of the amount and types of organic matter contributed to the Earth by interplanetary dust, in Space Life Sciences: Steps Toward Origin(S) of Life, ed. by M.P. Bernstein, M. Kress, R. NavarroGonzalez (Pergamon, Kidlington, 2004), pp. 57-66

J.M. Friedrich, H.L. McLain, J.P. Dworkin, D.P. Glavin, W.H. Towbin, M. Hill, D.S. Ebel, Effect of polychromatic X-ray microtomography imaging on the amino acid content of the Murchison CM chondrite. Meteorit. Planet. Sci. 54, 220-228 (2019)

M. Fries, J. Fries, Doppler weather radar as a meteorite recovery tool. Meteorit. Planet. Sci. 45, 1476-1487 (2010)

M. Fries, C. Laird, M. Hankey, J. Fries, R. Matson, V. Reddy, Estimation of meteorite fall mass and other properties from weather radar data, in 80th Annual Meeting of the Meteoritical Society, Abstract \#6251 (2017)

C.W. Gehrke, R.W. Zumwalt, W.A. Aue, D.L. Stalling, A. Duffield, K.A. Kvenvolden, C. Ponnamperuma, Carbon compounds in lunar fines from Mare Tranquillitatis; III, Organosiloxanes in hydrochloric acid hydrolysates, in Apollo 11 Lunar Science Conference, Proc., vol. 2 (Pergamon, New York, 1970), pp. 1845-1856 
C.W. Gehrke, R.W. Zumwalt, K. Kuo, W.A. Aue, D.L. Stalling, K.A. Kvenvolden, C. Ponnamperuma, Amino acid analyses of Apollo 14 samples, in Lunar Science Conference, 3rd, Proc., vol. 3 (Pergamon, Oxford, 1972), pp. 2119-2129

G.R. Gladstone, D.M. Hurley, K.D. Retherford, P.D. Feldman, W.R. Pryor, J.-Y. Chaufray, M. Versteeg, T.K. Greathouse, A.J. Steffl, H. Throop, J.W. Parker, D.E. Kaufmann, A.F. Egan, M.W. Davis, D.C. Slater, J. Mukherjee, P.F. Miles, A.R. Hendrix, A. Colaprete, S.A. Stern, LRO-LAMP observations of the LCROSS impact plume. Science 330, 472-476 (2010)

M.M. Grady, Meteorites from cold and hot deserts: How many, how big, and what sort, in Workshop on Extraterrestrial Materials from Cold and Hot Deserts. Lunar and Planetary Institute, Houston, Workshop on Extraterrestrial Materials from Cold and Hot Deserts, ed. by L.F.I.A. Schultz, A.M. Reid, M.E. Zolensky (2000), pp. 36-40

M.M. Grady, A.B. Verchovsky, I.A. Franchi, I.P. Wright, C.T. Pillinger, Light element geochemistry of the Tagish Lake CI2 chondrite; comparison with CI1 and CM2 meteorites. Meteorit. Planet. Sci. 37, 713735 (2002)

M. Grimaldo, Decontamination with cold plasma activated ionized hydrogen peroxide: Does it behave like a gas? in 60th Annual Biological Safety Conference, ABSA 1717B (2017)

M. Gritsevich, E. Lyytinen, J. Moilanen, T. Kohout, V. Dmitriev, V. Lupovka, V. Midtskogen, N. Kruglikov, A. Ischenko, G. Yakovlev, V. Grokhovsky, J. Haloda, P. Halodova, J. Peltoniemi, A. Aikkila, A. Taavitsainen, J. Lauanne, M. Pekkola, P. Kokko, P. Lahtinen, M. Larionov, First meteorite recovery based on observations by the Finnish Fireball Network, in Proceedings of the International Meteor Conference, Giron, France, 18-21 September 2014 (2014), pp. 162-169

E. Grosjean, G.A. Logan, Incorporation of organic contaminants into geochemical samples and an assessment of potential sources: Examples from Geoscience Australia marine survey S282. Org. Geochem. 38, 853869 (2007)

J. Guardiola, V. Rojo, G. Ramos, Influence of particle size, fluidization velocity and relative humidity on fluidized bed electrostatics. J. Electrost. 37, 1-20 (1995)

C.W. Haberle, L.A.J. Garvie, Extraterrestrial formation of oldhamite and portlandite through thermal metamorphism of calcite in the Sutter's Mill carbonaceous chondrite. Am. Mineral. 102, 2415-2421 (2017)

I. Halliday, A.T. Blackwell, A.A. Griffin, The Innisfree meteorite and the Canadian camera network. J. R. Astron. Soc. Can. 72, 15-39 (1978)

M. Hallworth, Rapid microbiological monitoring in pharmaceutical environments, in Environmental Monitoring: A Comprehensive Handbook, vol. 6, ed. by J. Moldenhaer (PDA, Bethesda, 2012), p. 93

T. Haltigin, C. Lange, R. Mugnolo, C. Smith (co-chairs), H. Amundsen, P. Bousquet, C. Conley, A. Debus, J. Dias, P. Falkner, V. Gass, A-M. Harri, E. Hauber, A.B. Ivanov, A.O. Ivanov, G. Kminek, O. Korablev, D. Koschny, J. Larranaga, B. Marty, S. McLennan, M. Meyer, E. Nilsen, P. Orleanski, R. Orosei, D. Rebuffat, F. Safa, N. Schmitz, S. Siljeström, N. Thomas, J. Vago, A-C. Vandaele, T. Voirin, C. Whetsel, Astrobiology (2018). https://doi.org/10.1089/ast.2018.29027.mars

R.D. Hanna, R.A. Ketcham, X-ray computed tomography of planetary materials: A primer and review of recent studies. Chem. Erde 77, 547-572 (2017)

P.E. Hare, K. Harada, S.W. Fox, Analyses for amino acids in lunar fines, in Apollo 11 Lunar Science Conference, Proc., vol. 2 (Pergamon, New York, 1970), pp. 1799-1803

R. Harvey, The origin and significance of Antarctic meteorites. Chem. Erde 63, 93-147 (2003)

W. Henderson, W.C. Kray, W.A. Newman, W.E. Reed, B.R. Simoneit, M. Calvin, Study of carbon compounds in Apollo 11 and Apollo 12 returned lunar samples, in Lunar Science Conference, 2nd, Proc., vol. 2 (Pergamon, Oxford, 1971), pp. 1901-1912

C.D.K. Herd, A. Blinova, D.N. Simkus, Y. Huang, R. Tarozo, C.M.O. Alexander, F. Gyngard, L.R. Nittler, G.D. Cody, M.L. Fogel, Y. Kebukawa, A.L.D. Kilcoyne, R.W. Hilts, G.F. Slater, D.P. Glavin, J.P. Dworkin, M.P. Callahan, J.E. Elsila, B. De Gregorio, R.M. Stroud, Origin and evolution of prebiotic organic matter as inferred from the Tagish Lake meteorite. Science 332, 1304-1307 (2011)

C.D.K. Herd, R.W. Hilts, A.W. Skelhorne, D.N. Simkus, Cold curation of pristine astromaterials: Insights from the Tagish Lake meteorite. Meteorit. Planet. Sci. 51, 499-519 (2016)

R.W. Hilts, C.D.K. Herd, D.N. Simkus, G.F. Slater, Soluble organic compounds in the Tagish Lake meteorite. Meteorit. Planet. Sci. 49, 526-549 (2014)

K.B. Hindley, M.A. Houlden, The British Fireball Network. Meteoritics 12, 257 (1977)

G.W. Hodgson, E. Bunnenberg, B. Halpern, E. Peterson, K.A. Kvenvolden, C. Ponnamperuma, Carbon compounds in lunar fines from Mare Tranquillitatis; II, Search for porphyrins, in Apollo 11 Lunar Science Conference, Proc., vol. 2 (Pergamon, New York, 1970), pp. 1829-1844

G.W. Hodgson, E. Bunnenberg, B. Halpern, E. Peterson, K.A. Kvenvolden, C. Ponnamperuma, Lunar pigments; porphyrin-like compounds from an Apollo 12 sample, in Lunar Science Conference, 2nd, Proc., vol. 2 (Pergamon, Oxford, 1971), pp. 1865-1874 
J.M.C. Holt, J.C. Bridges, J. Vrublevskis, F. Gaubert, Double walled isolator technology for Mars sample return facilities, in Proceedings of the 50th Lunar and Planetary Science Conference, Woodlands, TX, \#2408 (2019)

B. Hubad, A. Lapanje, The efficient method for simultaneous monitoring of the culturable as well as nonculturable airborne microorganisms. PLoS ONE 8, 9 (2013)

L.A. Hug, B.J. Baker, K. Anantharaman, C.T. Brown, A.J. Probst, C.J. Castelle, C.N. Butterfield, A.W. Hernsdorf, Y. Amano, K. Ise, Y. Suzuki, N. Dudek, D.A. Relman, K.M. Finstad, R. Amundson, B.C. Thomas, J.F. Banfield, A new view of the tree of life. Nat. Microbiol. 1, 6 (2016)

IEST-STD-CC1246E, Product cleanliness levels-Applications, requirements, and determination. Institute of Environmental Sciences and Technology, Schaumburg, IL, USA (2013)

ISO14698, Cleanrooms and associated controlled environments—Biocontamination control—Part 1: General Principles and Methods, ISO 14698-1:2003 (2003)

A. Jambon, Bronze Age iron: Meteoritic or not? A chemical strategy. J. Archaeol. Sci. 88, 47-53 (2017)

P. Jenniskens, M.D. Fries, Q.Z. Yin, M. Zolensky, A.N. Krot, S.A. Sandford, D. Sears, R. Beauford, D.S. Ebel, J.M. Friedrich, K. Nagashima, J. Wimpenny, A. Yamakawa, K. Nishiizumi, Y. Hamajima, M.W. Caffee, K.C. Welten, M. Laubenstein, A.M. Davis, S.B. Simon, P.R. Heck, E.D. Young, I.E. Kohl, M.H. Thiemens, M.H. Nunn, T. Mikouchi, K. Hagiya, K. Ohsumi, T.A. Cahill, J.A. Lawton, D. Barnes, A. Steele, P. Rochette, K.L. Verosub, J. Gattacceca, G. Cooper, D.P. Glavin, A.S. Burton, J.P. Dworkin, J.E. Elsila, S. Pizzarello, R. Ogliore, P. Schmitt-Kopplin, M. Harir, N. Hertkorn, A. Verchovsky, M. Grady, K. Nagao, R. Okazaki, H. Takechi, T. Hiroi, K. Smith, E.A. Silber, P.G. Brown, J. Albers, D. Klotz, M. Hankey, R. Matson, J.A. Fries, R.J. Walker, I. Puchtel, C.T.A. Lee, M.E. Erdman, G.R. Eppich, S. Roeske, Z. Gabelica, M. Lerche, M. Nuevo, B. Girten, S.P. Worden (Sutter's Mill Meteorite C.), Radarenabled recovery of the Sutter's Mill meteorite, a carbonaceous chondrite regolith breccia. Science 338, 1583-1587 (2012)

P. Jenniskens, J. Albers, C.E. Tillier, S.F. Edgington, R.S. Longenbaugh, S.J. Goodman, S.D. Rudlosky, A.R. Hildebrand, L. Hanton, F. Ciceri, R. Nowell, E. Lyytinen, D. Hladiuk, D. Free, N. Moskovitz, L. Bright, C.O. Johnston, E. Stern, Detection of meteoroid impacts by the Geostationary Lightning Mapper on the GOES-16 satellite. Meteorit. Planet. Sci. 53, 2445-2469 (2018)

L. Jimenez, Molecular applications to pharmaceutical processes and cleanroom environments. PDA J. Pharm. Sci. Technol. 65, 242-253 (2011)

A.J.G. Jurewicz, D.S. Burnett, R.C. Wiens, T.A. Friedmann, C.C. Hays, R.J. Hohlfelder, K. Nishiizumi, J.A. Stone, D.S. Woolum, R. Becker, A.L. Butterworth, A.J. Campbell, M. Ebihara, I.A. Franchi, V. Heber, C.M. Hohenberg, M. Humayun, K.D. McKeegan, K. McNamara, A. Meshik, R.O. Pepin, D. Schlutter, R. Wieler, The Genesis solar-wind collector materials. Space Sci. Rev. 105, 535-560 (2003)

Y. Kebukawa, S. Nakashima, T. Otsuka, K. Nakamura-Messenger, M.E. Zolensky, Rapid contamination during storage of carbonaceous chondrites prepared for micro FTIR measurements. Meteorit. Planet. Sci. 44, 545-557 (2009)

C.G. Keller, R.T. Howe, Hexsil tweezers for teleoperated micro-assembly, in Proceedings IEEE the Tenth Annual International Workshop on Micro Electro Mechanical Systems. An Investigation of Micro Structures, Sensors, Actuators, Machines and Robots (IEEE, Nagoya, 1997), pp. 72-77

W. Kinard, R. O'Neal, Long Duration Exposure Facility (LDEF) results, in 29th Aerospace Sciences Meeting, Abstract \#1971 (American Institute of Aeronautics and Astronautics, Washington, 1991)

B.V. King, I.V. Veryovkin, A.V. Zinovev, C.E. Tripa, M.J. Pellin, N. Toyoda, M. Schmeling, Ion beam removal of surface contamination in Genesis samples, in Lunar and Planetary Science Conference XXXXI, Abstract \#1975 (2010)

G.I. Kokhirova, J. Borovička, Observations of the 2009 Leonid activity by the Tajikistan fireball network. Astron. Astrophys. 533, 6 (2011)

K.R. Kuhlman, M.C. Rodriquez, C.P. Gonzalez, J.H. Allton, D.S. Burnett, Cleaning study of genesis sample 60487, in Lunar and Planetary Science Conference XXXXIV, Abstract \#2930 (2013)

K.A. Kvenvolden, S. Chang, J.W. Smith, J. Flores, K. Pering, C. Saxinger, F. Woeller, K. Keil, I. Breger, C. Ponnamperuma, Carbon compounds in lunar fines from Mare Tranquillitatis; I, Search for molecules of biological significance, in Apollo 11 Lunar Science Conference, Proc., vol. 2 (Pergamon, New York, 1970), pp. 1813-1828

K. Kwan, M. Cooper, M.T. La Duc, P. Vaishampayan, C. Stam, J.N. Benardini, G. Scalzi, C. MoisslEichinger, K. Venkateswaran, Evaluation of procedures for the collection, processing, and analysis of biomolecules from low-biomass surfaces. Appl. Environ. Microbiol. 77, 2943-2953 (2011)

M.T. La Duc, A. Dekas, S. Osman, C. Moissl, D. Newcombe, K. Venkateswaran, Isolation and characterization of bacteria capable of tolerating the extreme conditions of cleanroom environments. Appl. Environ. Microbiol. 73, 2600-2611 (2007)

M.T. La Duc, S. Osman, P. Vaishampayan, Y. Piceno, G. Andersen, J.A. Spry, K. Venkateswaran, Comprehensive census of bacteria in cleanrooms by using DNA microarray and cloning methods. Appl. Environ. Microbiol. 75, 6559-6567 (2009) 
M.T. La Duc, K. Venkateswaran, C.A. Conley, A genetic inventory of spacecraft and associated surfaces. Astrobiology 14, 15-23 (2014)

D.S. Lauretta, S.S. Balram-Knutson, E. Beshore, W.V. Boynton, C. Drouet d'Aubigny, D.N. DellaGiustina, H.L. Enos, D.R. Golish, C.W. Hergenrother, E.S. Howell, C.A. Bennett, E.T. Morton, M.C. Nolan, B. Rizk, H.L. Roper, A.E. Bartels, B.J. Bos, J.P. Dworkin, D.E. Highsmith, D.A. Lorenz, L.F. Lim, R. Mink, M.C. Moreau, J.A. Nuth, D.C. Reuter, A.A. Simon, E.B. Bierhaus, B.H. Bryan, R. Ballouz, O.S. Barnouin, R.P. Binzel, W.F. Bottke, V.E. Hamilton, K.J. Walsh, S.R. Chesley, P.R. Christensen, B.E. Clark, H.C. Connolly, M.K. Crombie, M.G. Daly, J.P. Emery, T.J. McCoy, J.W. McMahon, D.J. Scheeres, S. Messenger, K. Nakamura-Messenger, K. Righter, S.A. Sandford, OSIRIS-REx: Sample return from Asteroid (101955) Bennu. Space Sci. Rev. 212, 925-984 (2017)

L. Le Roy, K. Altwegg, H. Balsiger, J.-J. Berthelier, A. Bieler, C. Briois, U. Calmonte, M.R. Combi, J. De Keyser, F. Dhooghe, B. Fiethe, S.A. Fuselier, S. Gasc, T.I. Gombosi, M. Hässig, A. Jäckel, M. Rubin, C.-Y. Tzou, Inventory of the volatiles on comet $67 \mathrm{P} /$ Churyumov-Gerasimenko from Rosetta/ROSINA. Astron. Astrophys. 583, A1 (2015)

S.R. Lipsky, R.J. Cushley, C.G. Horvath, W.J. McMurray, Analysis of lunar material for organic compounds, in Apollo 11 Lunar Science Conference, Proc., vol. 2 (Pergamon, New York, 1970), pp. 1871-1873

J.T. Lonsdale, The Pena Blanca Spring meteorite, Brewster County, Texas. Am. Mineral. 32, 354-364 (1947)

M.D.J. Lynch, J.D. Neufeld, Ecology and exploration of the rare biosphere. Nat. Rev. Microbiol. 13, 217-229 (2015)

A. Mahnert, P. Vaishampayan, A.J. Probst, A. Auerbach, C. Moissl-Eichinger, K. Venkateswaran, G. Berg, Cleanroom maintenance significantly reduces abundance but not diversity of indoor microbiomes. PLoS ONE 10, 20 (2015)

P.M. Martin, A.A. Mills, Size and shape of chondrules in the Bjurböle and Chainpur meteorites. Earth Planet. Sci. Lett. 33, 239-248 (1976)

U.B. Marvin, Meteorites in history: An overview from the Renaissance to the 20th century. Geol. Soc. (Lond.) Spec. Publ. 256, 15-71 (2006)

S. Matsusaka, H. Maruyama, T. Matsuyama, M. Ghadiri, Triboelectric charging of powders: A review. Chem. Eng. Sci. 65, 5781-5807 (2010)

G.J.H. McCall, A.J. Bowden, R.J. Howarth, The history of meteoritics-Overview. Geol. Soc. (Lond.) Spec. Publ. 256, 1-13 (2006)

T.J. McCoy, Hopewell meteoritic metal beads: Clues to trade 2,000 years ago. Elements 14, 360-361 (2018)

T.J. McCoy, A.E. Marquardt, J.T. Wasson, R.D. Ash, E.P. Vicenzi, The Anoka, Minnesota iron meteorite as parent to Hopewell meteoritic metal beads from Havana, Illinois. J. Archaeol. Sci. 81, 13-22 (2017)

J.C. McLane, E.A. King, D.A. Flory, K.A. Richardson, J.P. Dawson, W.W. Kemmerer, Lunar receiving laboratory. Science 155, 525-529 (1967)

S.M. McLennan, M.A. Sephton, C. Allen, A.C. Allwood, R. Barbieri, D.W. Beaty, P. Boston, M. Carr, M. Grady, J. Grant, V.S. Heber, C.D.K. Herd, B. Hofmann, P. King, N. Mangold, G.G. Ori, A.P. Rossi, F. Raulin, S.W. Ruff, B. Sherwood Lollar, S. Symes, M.G. Wilson, Planning for Mars returned sample science: Final report of the MSR End-to-End International Science Analysis Group (E2E-iSAG). Astrobiology 12, 175-230 (2011)

K.M. McNamara, E.K. Stansbery, Analysis of molecular contamination on genesis collectors through spectroscopic ellipsometry, in 36th Lunar and Planetary Science Conference, Abstract \#2402 (2005)

R.M. Mecikalski, L.D. Carey, Radar reflectivity and altitude distributions of lightning flashes as a function of three main storm types. J. Geophys. Res., Atmos. 123, 12814-12828 (2018)

W.G. Meinschein, T.J. Jackson, J.M. Mitchell, E. Cordes, V.J. Shiner Jr., Search for alkanes of 15-30 carbon atom length in lunar fines, in Apollo 11 Lunar Science Conference, Proc., vol. 2 (Pergamon, New York, 1970), pp. 1875-1877

S. Messenger, Opportunities for the stratospheric collection of dust from short-period comets. Meteorit. Planet. Sci. 37, 1491-1505 (2002)

S. Messenger, K. Nakamura-Messenger, L.P. Keller, S.J. Clemett, Pristine stratospheric collection of interplanetary dust on an oil-free polyurethane foam substrate. Meteorit. Planet. Sci. 50, 1468-1485 (2015)

E.T. Mickelson, Cleaning and Cleanliness Verification Techniques for Mars Returned Sample Handling. An MRSH Document, NASA JSC 29738 (2002a)

E.T. Mickelson, Molecular Contamination Control: A Unified Cleaning and Verification Strategy for a Sample Receiving Facility. An MRSH Document, NASA JSC 29689 (2002b)

MIL-STD-1246C, Military Standard: Product Cleanliness Levels and Contamination Control Program. Department of Defense (1994)

S.D. Miller, W.C. Straka, A.S. Bachmeier, T.J. Schmit, P.T. Partain, Y.J. Noh, Earth-viewing satellite perspectives on the Chelyabinsk meteor event. Proc. Natl. Acad. Sci. 110, 18092-18097 (2013)

J.J. Minich, Q.Y. Zhu, S. Janssen, R. Hendrickson, A. Amir, R. Vetter, J. Hyde, M.M. Doty, K. Stillwell, J. Benardini, J.H. Kim, E.E. Allen, K. Venkateswaran, R. Knight, KatharoSeq enables high-throughput microbiome analysis from low-biomass samples. mSystems 3, 16 (2018) 
K.R. Mitchell, C.D. Takacs-Vesbach, A comparison of methods for total community DNA preservation and extraction from various thermal environments. J. Ind. Microbiol. Biotech. 35, 1139-1147 (2008)

C. Moissl, J.C. Bruckner, K. Venkateswaran, Archaeal diversity analysis of spacecraft assembly cleanrooms. ISME J. 2, 115-119 (2008)

C. Moissl-Eichinger, Archaea in artificial environments: Their presence in global spacecraft cleanrooms and impact on planetary protection. ISME J. 5, 209-219 (2011)

C. Moissl-Eichinger, A.K. Auerbach, A.J. Probst, A. Mahnert, L. Tom, Y. Piceno, G.L. Andersen, K. Venkateswaran, P. Rettberg, S. Barczyk, R. Pukall, G. Berg, Quo vadis? Microbial profiling revealed strong effects of cleanroom maintenance and routes of contamination in indoor environments. Sci. Rep. 5, 13 (2015)

M. Mora, A. Mahnert, K. Koskinen, M.R. Pausan, L. Oberauner-Wappis, R. Krause, A.K. Perras, G. Gorkiewicz, G. Berg, C. Moissi-Eichinger, Microorganisms in confined habitats: Microbial monitoring and control of intensive care units, operating rooms, cleanrooms and the international space station. Front. Microbiol. 7, 20 (2016)

M.E. Murphy, V.E. Modzeleski, B. Nagy, W.M. Scott, M. Young, C.M. Drew, P.B. Hamilton, H.C. Urey, Analysis of Apollo 11 lunar samples by chromatography and mass spectrometry; pyrolysis products, hydrocarbons, sulfur, amino acids, in Apollo 11 Lunar Science Conference, Proc., vol. 2 (Pergamon, New York, 1970), pp. 1879-1890

T. Nakamura, T. Noguchi, A. Tsuchiyama, H. Yurimoto, K. Nagao, M. Ebihara, R. Okazaki, F. Kitajima, H. Naraoka, M. Abe, A. Fujimura, T. Yada, T. Okada, Y. Ishibashi, K. Shirai, T. Mukai, H. Yano, T. Yamada, H. Kuninaka, M. Yoshikawa, J. Kawaguchi, The first recovery of asteroidal samples by the Hayabusa mission, in AGU Fall Meeting Abstracts (2011)

K. Nakamura-Messenger, S. Messenger, L.P. Keller, S.J. Clemett, M.E. Zolensky, Organic globules in the Tagish Lake meteorite: Remnants of the protosolar disk. Science 314, 1439-1442 (2006)

H. Naraoka, H. Mita, K. Hamase, M. Mita, H. Yabuta, K. Saito, K. Fukushima, F. Kitajima, S.A. Sandford, T. Nakamura, T. Noguchi, R. Okazaki, K. Nagao, M. Ebihara, H. Yurimoto, A. Tsuchiyama, M. Abe, K. Shirai, M. Ueno, T. Yada, Y. Ishibashi, T. Okada, A. Fujimura, T. Mukai, M. Yoshikawa, J. Kawaguchi, Preliminary organic compound analysis of microparticles returned from Asteroid 25143 Itokawa by the Hayabusa mission. Geochem. J. 46, 61-72 (2012)

NASA, in Summer Conference on Lunar Exploration and Science, Falmouth, Massachusetts, July 19-31, 1965 (Scientific and Technical Information Division, National Aeronautics and Space Administration, Washington, 1965), $421 \mathrm{pp}$.

NASA, in Handbook for the Microbial Examination of Space Hardware. NASA Technical Handbook, NASAHDBK-6022 (2010), $52 \mathrm{pp.}$

NASA, in Planetary Protection Provisions for Robotic Extraterrestrial Missions, NASA Interim Directive, NID 8020.109A (2017), 49 pp.

N.D. Novikova, Review of the knowledge of microbial contamination of the Russian manned spacecraft. Microb. Ecol. 47, 127-132 (2004)

J. Oberst, S. Molau, D. Heinlein, C. Gritzner, M. Schindler, P. Spurny, Z. Ceplecha, J. Rendtel, H. Betlem, The "European Fireball Network": Current status and future prospects. Meteorit. Planet. Sci. 33, 49-56 (1998)

R. Okazaki, K. Nagao, Y.N. Miura, T. Osawa, K. Bajo, S. Matsuda, T. Nakamura, K. Shirai, M. Abe, T. Yada, T. Noguchi, Y. Ishibashi, A. Fujimura, T. Mukai, M. Ueno, T. Okada, M. Yoshikawa, J. Kawaguchi, Noble gases recovered from the Hayabusa sample container, in Lunar and Planetary Science Conference (2011) p. 1653

R. Okazaki, H. Sawada, S. Yamanouchi, S. Tachibana, Y. Miura, K. Sakamoto, Y. Takano, M. Abe, S. Itoh, K. Yamada, H. Yabuta, C. Okamoto, H. Yano, T. Noguchi, T. Nakamura, K. Nagao, Hayabusa2 sample catcher and container: Metal-seal system for vacuum encapsulation of returned samples with volatiles and organic compounds recovered from C-type asteroid Ryugu. Space Sci. Rev. 208, 107-124 (2017)

J. Oro, W.S. Updegrove, J. Gibert, J. McReynolds, E. Gil-Av, J. Ibanez, A. Zlatkis, D.A. Flory, R.L. Levy, C.J. Wolf, Organogenic elements and compounds in type C and D lunar samples from Apollo 11, in Apollo 11 Lunar Science Conference, Proc., vol. 2 (Pergamon, New York, 1970), pp. 1901-1920

V.I. Oyama, E.L. Merek, M.P. Silverman, A search for viable organisms in a lunar sample, in Apollo 11 Lunar Science Conference, Proc., vol. 2 (Pergamon, New York, 1970), pp. 1921-1927

V.I. Oyama, E.L. Merek, M.P. Silverman, C.W. Boylen, Search for viable organisms in lunar samples; further biological studies on Apollo 11 core, Apollo 12 bulk, and Apollo 12 core samples, in Lunar Science Conference, 2nd, Proc., vol. 2 (Pergamon, Oxford, 1971), pp. 1931-1937

D. Papanastassiou, J.M.D. Day, D.P. Glavin, G.R. Huss, R.L. Korotev, L.E. Nyquist, M. Wadhwa, T.J. Zega, Lunar Curation Task Force Report. NASA report to the Curation and Analysis Planning Team for Extraterrestrial Materials (2015), p. 14 
K.E. Peters, C.C. Walters, J.M. Moldowan, The Biomarker Guide: Volume 1. Biomarkers and Isotopes in the Environment and Human History (Cambridge University Press, Cambridge, 2005), 492 pp.

O.P. Popova, P. Jenniskens, V. Emel'yanenko, A. Kartashova, E. Biryukov, S. Khaibrakhmanov, V. Shuvalov, Y. Rybnov, A. Dudorov, V.I. Grokhovsky, D.D. Badyukov, Q.Z. Yin, P.S. Gural, J. Albers, M. Granvik, L.G. Evers, J. Kuiper, V. Kharlamov, A. Solovyov, Y.S. Rusakov, S. Korotkiy, I. Serdyuk, A.V. Korochantsev, M.Y. Larionov, D. Glazachev, A.E. Mayer, G. Gisler, S.V. Gladkovsky, J. Wimpenny, M.E. Sanborn, A. Yamakawa, K.L. Verosub, D.J. Rowland, S. Roeske, N.W. Botto, J.M. Friedrich, M.E. Zolensky, L. Le, D. Ross, K. Ziegler, T. Nakamura, I. Ahn, J.I. Lee, Q. Zhou, X.H. Li, Q.L. Li, Y. Liu, G.Q. Tang, T. Hiroi, D. Sears, I.A. Weinstein, A.S. Vokhmintsev, A.V. Ishchenko, P. Schmitt-Kopplin, N. Hertkorn, K. Nagao, M.K. Haba, M. Komatsu, T. Mikouchi, C. Chelyabinsk Airburst, Chelyabinsk airburst, damage assessment, meteorite recovery, and characterization. Science 342, 1069-1073 (2013)

G. Preti, R.C. Murphy, K. Biemann, The search for organic compounds in various Apollo 12 samples by mass spectrometry, in Lunar Science Conference, 2nd, Proc., vol. 2 (Pergamon, Oxford, 1971), pp. 1879-1889

M. Prinz, K. Keil, P.F. Hlava, J.L. Berkley, C.B. Gomes, W.S. Curvello, Studies of Brazilian Meteorites: 3. Origin and history of Angra-Dos-Reis achondrite. Earth Planet. Sci. Lett. 35, 317-330 (1977)

A. Probst, P. Vaishampayan, S. Osman, C. Moissl-Eichinger, G.L. Andersen, K. Venkateswaran, Diversity of anaerobic microbes in spacecraft assembly cleanrooms. Appl. Environ. Microbiol. 76, 2837-2845 (2010)

M. Ramstorp, Introduction to Contamination Control and Cleanroom Technology (Wiley, New York, 2000)

M.S. Rappe, S.J. Giovannoni, The uncultured microbial majority. Annu. Rev. Microbiol. 57, 369-394 (2003)

J.S. Raval, E. Koch, A.D. Donnenberg, Real-time monitoring of non-viable airborne particles correlates with airborne colonies and represents an acceptable surrogate for daily assessment of cell-processing cleanroom performance. Cytotherapy 14, 1144-1150 (2012)

J.A. Reuter, D.V. Spacek, M.P. Snyder, High-throughput sequencing technologies. Mol. Cell 58, 586-597 (2015)

M.A. Reynolds, N.L. Turner, J.C. Hurgeton, M.F. Barbee, D.A. Flory, B.R. Simoneit, Environmental control of lunar samples in the lunar receiving laboratory, in Analytical Methods Developed for Application to Lunar Samples Analyses, American Society for Testing and Materials, ASTM STP, vol. 539 (1973), pp. 3-15

J.H. Rho, A.J. Bauman, T.F. Yen, J. Bonner, Fluorometric examination of the returned lunar fines from Apollo 11, in Apollo 11 Lunar Science Conference, Proc., vol. 2 (Pergamon, New York, 1970), pp. 1929-1932

J.H. Rho, A.J. Bauman, J. Bonner, Absence of porphyrins in an Apollo 12 lunar surface sample, in Lunar Science Conference, 2nd, Proc., vol. 2 (Pergamon, Oxford, 1971), pp. 1875-1877

J.H. Rho, E.A. Cohen, A.J. Bauman, Spectrofluorometric search for porphyrins in Apollo 14 surface fines, in Lunar Science Conference, 3rd, Proc., vol. 3 (Pergamon, Oxford, 1972), pp. 2149-2155

K. Righter, C.M. Corrigan, T.J. McCoy, R.P. Harvey, 35 Seasons of US Antarctic Meteorites (Am. Geophys. Union, Washington, 2014)

A.J. Rissanen, E. Kurhela, T. Aho, T. Oittinen, M. Tiirola, Storage of environmental samples for guaranteeing nucleic acid yields for molecular microbiological studies. Appl. Microbiol. Biotechnol. 88, 977-984 (2010)

B. Robert, A low-power micromanipulator and microdissector. J. Sci. Instrum. 28, 65 (1951)

H.L. Rose, C.A. Dewey, M.S. Ely, S.L. Willoughby, T.M. Parsons, V. Cox, P.M. Spencer, S.A. Weller, Comparison of eight methods for the extraction of bacillus atrophaeus spore DNA from eleven common interferents and a common swab. PLoS ONE 6, e22668 (2011)

J. Rummel, M. Race, D. DeVincenzi, P. Schad, P. Stabekis, M. Viso, S. Acevedo, A Draft Test Protocol for Detecting Possible Biohazards in Martian Samples Returned to Earth (2002). NASA technical publication 211842

V.E. Sakraida, Cleanroom Design in 10 Easy Steps, Engineered Systems Magazine, Troy, MI (2008)

S.A. Sandford, D.E. Brownlee, Response to comment on "Organics captured from Comet 81P/Wild 2 by the Stardust spacecraft". Science 317, 1 (2007)

S.A. Sandford, J. Aleon, C.M.O. Alexander, T. Araki, S. Bajt, G.A. Baratta, J. Borg, J.P. Bradley, D.E. Brownlee, J.R. Brucato, M.J. Burchell, H. Busemann, A. Butterworth, S.J. Clemett, G. Cody, L. Colangeli, G. Cooper, L. D’Hendecourt, Z. Djouadi, J.P. Dworkin, G. Ferrini, H. Fleckenstein, G.J. Flynn, I.A. Franchi, M. Fries, M.K. Gilles, D.P. Glavin, M. Gounelle, F. Grossemy, C. Jacobsen, L.P. Keller, A.L.D. Kilcoyne, J. Leitner, G. Matrajt, A. Meibom, V. Mennella, S. Mostefaoui, L.R. Nittler, M.E. Palumbo, D.A. Papanastassiou, F. Robert, A. Rotundi, C.J. Snead, M.K. Spencer, F.J. Stadermann, A. Steele, T. Stephan, P. Tsou, T. Tyliszczak, A.J. Westphal, S. Wirick, B. Wopenka, H. Yabuta, R.N. Zare, M.E. Zolensky, Organics captured from comet 81P/Wild 2 by the Stardust spacecraft. Science 314, 17201724 (2006) 
S.A. Sandford, S. Bajt, S.J. Clemett, G.D. Cody, G. Cooper, B.T. Degregorio, V. de Vera, J.P. Dworkin, J.E. Elsila, G.J. Flynn, D.P. Glavin, A. Lanzirotti, T. Limero, M.P. Martin, C.J. Snead, M.K. Spencer, T. Stephan, A. Westphal, S. Wirick, R.N. Zare, M.E. Zolensky, Assessment and control of organic and other contaminants associated with the Stardust sample return from comet 81P/Wild 2. Meteorit. Planet. Sci. 45, 406-433 (2010)

T. Sandle, Environmental monitoring: A practical approach, in Environmental Monitoring: A Comprehensive Handbook, vol. 6 (2012), pp. 29-54

T. Sandle, K. Skinner, Study of psychrophilic and psychrotolerant micro-organisms isolated in cold rooms used for pharmaceutical processing. J. Appl. Microbiol. 114, 1166-1174 (2013)

E.K. Sansom, P. Bland, J. Paxman, M. Towner, A novel approach to fireball modeling: The observable and the calculated. Meteorit. Planet. Sci. 50, 1423-1435 (2015)

H. Sawada, R. Okazaki, S. Tachibana, K. Sakamoto, Y. Takano, C. Okamoto, H. Yano, Y. Miura, M. Abe, S. Hasegawa, T. Noguchi, Hayabusa2 sampler: Collection of asteroidal surface material. Space Sci. Rev. 208, 81-106 (2017)

M. Schmeling, D.S. Burnett, J.H. Allton, M. Rodriquez, C.E. Tripa, I.V. Veryovkin, Application of CO2 snow jet cleaning in conjunction with laboratory based total reflection X-ray fluorescence, in Lunar and Planetary Science Conference XXXXIV, Abstract \#2465 (2013)

P. Schwendner, C. Moissl-Eichinger, S. Barczyk, M. Bohmeier, R. Pukall, P. Rettberg, Insights into the microbial diversity and bioburden in a South American spacecraft assembly cleanroom. Astrobiology 13, 1140-1154 (2013)

D.W.G. Sears, H. Sears, D.S. Ebel, S. Wallace, J.M. Friedrich, X-ray computed tomography imaging: A notso-nondestructive technique. Meteorit. Planet. Sci. 51, 833-838 (2016)

D.W.G. Sears, A. Sehlke, J.M. Friedrich, M.L. Rivers, D.S. Ebel, X-ray computed tomography of extraterrestrial rocks eradicates their natural radiation record and the information it contains. Meteorit. Planet. Sci. 53, 2624-2631 (2018)

L.S. Sherman, J.R. Waldbauer, R.E. Summons, Improved methods for isolating and validating indigenous biomarkers in Precambrian rocks. Org. Geochem. 38, 1987-2000 (2007)

Y. Shiba, C. Shimoda, T. Maruyama, S. Okumura, M. Tomita, A. Murasawa, K. Ohtsuka, H. Tomioka, E. Hidaka, Photographic observations of the 1996 Leonid fireballs in Japan. Earth Moon Planets 77, 47-54 (1997)

S. Shokralla, J.L. Spall, J.F. Gibson, M. Hajibabaei, Next-generation sequencing technologies for environmental DNA research. Mol. Ecol. 21, 1794-1805 (2012)

B.R. Simoneit, D.A. Flory, Apollo 11, 12, and 13 Organic Contamination Monitoring History. Lunar and Earth Sciences Division Internal Note, NASA Document MSC-04350 (1971)

B.R. Simoneit, D.A. Flory, M.A. Reynolds, Organic contamination monitoring and control in the lunar receiving laboratory, in Analytical Methods Developed for Application to Lunar Samples Analyses, American Society for Testing and Materials, ASTM STP, vol. 539 (1973), pp. 16-34

M.K. Spencer, R.N. Zare, Comment on "Organics Captured from Comet 81P/Wild 2 by the Stardust Spacecraft". Science 317, 1680 (2007)

M.K. Spencer, S.J. Clemett, S.A. Sandford, D.S. McKay, R.N. Zare, Organic compound alteration during hypervelocity collection of carbonaceous materials in aerogel. Meteorit. Planet. Sci. 44, 15-24 (2009)

E.K. Stansbery, K.M. McNamara, Genesis preliminary examination-Ellipsometry overview, in 42nd Lunar and Planetary Science Conference, Abstract \#2145 (2005)

E.K. Stansbery, G.R.P. Team, Genesis recovery processing, in 36th Lunar and Planetary Science Conference, Abstract \#2179 (2005)

A. Steele, F.M. McCubbin, M. Fries, M. Glamoclija, L. Kater, H. Nekvasil, Graphite in an Apollo 17 impact melt breccia. Science 329, 51 (2010)

D. Stuart, D. Eagleson, R. Lloyd, C. Hersey, D. Eagleson, Analysis of the class III biological safety cabinet integrity test. Appl. Biosafety 17, 128-131 (2012)

G.M. Sullivan, D.I. Klebe, Searching for fireballs, in American Astronomical Society Meeting, Abstracts \#204 (2004)

G.R. Taylor, W. Ellis, P.H. Johnson, K. Kropp, T. Groves, Microbial assay of lunar samples, in Lunar Science Conference, 2nd, Proc., vol. 2 (Pergamon, Oxford, 1971), pp. 1939-1948

M. Tessler, J.S. Neumann, E. Afshinnekoo, M. Pineda, R. Hersch, L.F.M. Velho, B.T. Segovia, F.A. LansacToha, M. Lemke, R. DeSalle, C.E. Mason, M.R. Brugler, Large-scale differences in microbial biodiversity discovery between $16 \mathrm{~S}$ amplicon and shotgun sequencing. Sci. Rep. 7, 14 (2017)

S.M. Tikoo, B.P. Weiss, W.S. Cassata, D.L. Shuster, J. Gattacceca, E.A. Lima, C. Suavet, F. Nimmo, M.D. Fuller, Decline of the lunar core dynamo. Earth Planet. Sci. Lett. 404, 89-97 (2014)

J. Toporski, A. Steele, Observations from a 4-year contamination study of a sample depth profile through the martian meteorite Nakhla. Astrobiology 7, 389-401 (2007) 
J. Tremblay, K. Singh, A. Fern, E.S. Kirton, S.M. He, T. Woyke, J. Lee, F. Chen, J.L. Dangl, S.G. Tringe, Primer and platform effects on 16S rRNA tag sequencing. Front. Microbiol. 6 (2015)

J.M. Trigo-Rodriguez, J. Llorca, A.J. Castro-Tirado, J.L. Ortiz, J.A. Docobo, J. Fabregat, The Spanish fireball network. Astron. Geophys. 47, 26-28 (2006)

P. Tsou, D.E. Brownlee, S.A. Sandford, F. Horz, M.E. Zolensky, Wild 2 and interstellar sample collection and Earth return. J. Geophys. Res., Planets 108, 21 (2003)

USP, Microbiological Evaluation of Cleanrooms and Other Controlled Environments. USP (United States Pharmacopeia) General Chapter $<1116>$, in USP 36 (United States Pharmacopeia Convention, North Bethesda, 2013) (2013), http://ftp.uspbpep.com/v29240/usp29nf24s0_c1116.html. Accessed 24 April 2019

P. Vaishampayan, A.J. Probst, M.T. La Duc, E. Bargoma, J.N. Benardini, G.L. Andersen, K. Venkateswaran, New perspectives on viable microbial communities in low-biomass cleanroom environments. ISME J. 7, 312-324 (2013)

K.E. Vander Kaaden, F.M. McCubbin, P.K. Byrne, N.L. Chabot, C.M. Ernst, C.L. Johnson, M.S. Thompson, Revolutionizing our understanding of the Solar System via sample return from Mercury. Space Sci. Rev. (2019, in press). https://doi.org/10.1007/s11214-019-0614-X

D. Vaniman, B.M. French, H.H. Schmitt, G.H. Heiken, Lunar Sourcebook; A User's Guide to the Moon (Cambridge University Press, Cambridge, 1991)

J.B. Vaught, M.K. Henderson, in Biological Sample Collection, Processing, Storage and Information Management. IARC Sci. Publ., vol. 163 (2011), pp. 23-42

K. Venkateswaran, N. Hattori, M.T. La Duc, R. Kern, ATP as a biomarker of viable microorganisms in cleanroom facilities. J. Microbiol. Methods 52, 367-377 (2003)

D. Venton, Inner workings: Networks of cameras are tracking meteorites with unprecedented precision. Proc. Natl. Acad. Sci. 114, 7472 (2017)

T. Větrovský, P. Baldrian, The variability of the $16 \mathrm{~S}$ rRNA gene in bacterial genomes and its consequences for bacterial community analyses. PLoS ONE 8, 10 (2013)

J. Veverka, Comet Surface Sample Return (CSSR) Mission Concept Study (2010a), pp. 1-33

J. Veverka, Cryogenic Comet Nucleus Sample Return (CNSR) Mission Technology Study (2010b), pp. 1-45

J. Vrublevskis, L. Berthoud, Y. McColluch, P. Bowman, J. Holt, J. Bridges, A. Bennett, F. Gaubert, L. Duvet, Description of European Space Agency (ESA) double walled isolator (DWI) breadboard currently under development for demonstration of critical technology foreseen to be used in the Mars sample receiving facility (MSRF), in Proceedings of the Second International Mars Sample Return Conference, Berlin, Germany, \#6009 (2018a)

J. Vrublevskis, S. Duncan, L. Berthoud, P. Bowman, R. Hills, Y. McCulloch, D. Pisla, C. Vaida, B. Gherman, M. Hofbaur, B. Dieber, N. Neythalath, C. Smith, M. van Winnendael, L. Duvet, Description of European Space Agency (ESA) remote manipulator (RM) system breadboard currently under development for demonstration of critical technology foreseen to be used in the Mars sample receiving facility (MSRF), in Proceedings of the Second International Mars Sample Return Conference, Berlin, Germany, \#6010 (2018b)

R.J. Walker, M.F. Horan, C.K. Shearer, J.J. Papike, Low abundances of highly siderophile elements in the lunar mantle: Evidence for prolonged late accretion. Earth Planet. Sci. Lett. 224, 399-413 (2004)

W. Watson, Enhancements to the Sentinel fireball network video software, in Society for Astronomical Sciences Annual Symposium, vol. 28 (2009), p. 43

$\mathrm{R}$. Webb, A fast track to zero environmental pathogens using novel ionized hydrogen peroxide technology. Infection Control Today. February 1 issue (2011)

T. Weinmaier, A.J. Probst, M.T. La Duc, D. Ciobanu, J.F. Cheng, N. Ivanova, T. Rattei, P. Vaishampayan, A viability-linked metagenomic analysis of cleanroom environments: Eukarya, prokaryotes, and viruses. Microbiome 3, 14 (2015)

R.J. Weryk, P.G. Brown, A. Domokos, W.N. Edwards, Z. Krzeminski, S.H. Nudds, D.L. Welch, The southern Ontario all-sky meteor camera network, in Advances in Meteoroid and Meteor Science, ed. by J.M. Trigo-Rodríguez, F.J.M. Rietmeijer, J. Llorca, D. Janches (Springer, New York, 2008), pp. 241-246

A.J. Westphal, R.K. Bastien, J. Borg, J.C. Bridges, D.E. Brownlee, M.J. Burchell, A.F. Cheng, B.C. Clark, Z. Djouadi, C. Floss, I. Franchi, Z. Gainsforth, G.A. Graham, S.F. Green, P.R. Heck, M. Horanyi, P. Hoppe, F.P. Hoerz, J. Huth, A.T. Kearsley, H. Leroux, K. Marhas, K. Nakamura-Messenger, S.A. Sandford, T.H. See, F.J. Stadermann, N.E. Teslich, S. Tsitrin, J.L. Warren, P.J. Wozniakiewicz, M.E. Zolensky, Discovery of non-random spatial distribution of impacts in the Stardust cometary collector. Meteorit. Planet. Sci. 43, 415-429 (2008)

G.W. Wetherill, D.O. Revelle, Which fireballs are meteorites? A study of the Prairie Network photographic meteor data. Icarus 48, 308-328 (1981)

A.F. Whitaker, D. Dooling, LDEF materials results for spacecraft applications: Executive summary. NASA STI/Recon Technical Report N (1995), p. 23549 
D.R. White, Lunar sample Processing in the Lunar Receiving Laboratory High-Vacuum Complex. Apollo Experience Report, NASA Technical Note D-8298, Johnson Space Center, Houston, TX (1976)

W. Whyte, Cleanroom Design, 2nd edn. (Wiley, New York, 2001)

W. Whyte, Cleanroom Technology: Fundamentals of Design, Testing and Operation, 2nd edn. (Wiley, New York, 2010)

M. Wiśniewski, P. Zoladek, A. Olech, Z. Tyminski, M. Maciejewski, K. Fietkiewicz, R. Rudawska, M. Gozdalski, M.P. Gawronski, T. Suchodolski, M. Myszkiewicz, M. Stolarz, K. Polakowski, Current status of Polish Fireball Network. Planet. Space Sci. 143, 12-20 (2017)

I.P. Wright, S.S. Russell, S.R. Boyd, C. Meyer, C.T. Pillinger, Xylan: A potential contaminant for lunar samples and Antarctic meteorites, in Proceedings of the 22nd Lunar and Planetary Science Conference, vol. 22 (1992), pp. 449-458

T. Yada, A. Fujimura, M. Abe, T. Nakamura, T. Noguchi, R. Okazaki, K. Nagao, Y. Ishibashi, K. Shirai, M.E. Zolensky, S. Sandford, T. Okada, M. Uesugi, Y. Karouji, M. Ogawa, S. Yakame, M. Ueno, T. Mukai, M. Yoshikawa, J. Kawaguchi, Hayabusa-returned sample curation in the planetary material sample curation facility of JAXA. Meteorit. Planet. Sci. 49, 135-153 (2014)

Y. Zhang, C-x. Xin, X. Wang, Y.-L. Deng, Detection of microorganism from China's spacecraft assembly cleanroom. Acta Astronaut. (2018, in press). https://doi.org/10.1016/j.actaastro.2018.08.024

S. Zielińska, P. Radkowski, A. Blendowska, A. Ludwig-Galezowska, J.M. Los, M. Los, The choice of the DNA extraction method may influence the outcome of the soil microbial community structure analysis. MicrobiologyOpen 6, 11 (2017)

K.-P. Zimmer, Optical designs for stereomicroscopes, in International Optical Design Conference (SPIE, Bellingham, 1998), p. 8

B. Zohuri, Physics of Cryogenics: An Ultralow Temperature Phenomenon (Elsevier, Amsterdam, 2017)

M.E. Zolensky, T. Girard, Stardust Spacecraft Program Contamination Control Plan. Astromaterials Research and Exploration Science, NASA Johnson Space Center, JSC-27954 (1997), p. 26

M.E. Zolensky, K. Nakamura, M. Gounelle, T. Mikouchi, T. Kasama, O. Tachikawa, E. Tonui, Mineralogy of Tagish Lake; an ungrouped type 2 carbonaceous chondrite. Meteorit. Planet. Sci. 37, 737-761 (2002)

M. Zolensky, P. Bland, P. Brown, I. Halliday, Flux of extraterrestrial materials, in Meteorites and the Early Solar System II, ed. by D.S. Lauretta, H.Y. McSween (University of Arizona Press, Tucson, 2006a), pp. 869-888

M.E. Zolensky, T.J. Zega, H. Yano, S. Wirick, A.J. Westphal, M.K. Weisberg, I. Weber, J.L. Warren, M.A. Velbel, A. Tsuchiyama, P. Tsou, A. Toppani, N. Tomioka, K. Tomeoka, N. Teslich, M. Taheri, J. Susini, R. Stroud, T. Stephan, F.J. Stadermann, C.J. Snead, S.B. Simon, A. Simionovici, T.H. See, F. Robert, F.J.M. Rietmeijer, W. Rao, M.C. Perronnet, D.A. Papanastassiou, K. Okudaira, K. Ohsumi, I. Ohnishi, K. Nakamura-Messenger, T. Nakamura, S. Mostefaoui, T. Mikouchi, A. Meibom, G. Matrajt, M.A. Marcus, H. Leroux, L. Lemelle, L. Le, A. Lanzirotti, F. Langenhorst, A.N. Krot, L.P. Keller, A.T. Kearsley, D. Joswiak, D. Jacob, H. Ishii, R. Harvey, K. Hagiya, L. Grossman, J.N. Grossman, G.A. Graham, M. Gounelle, P. Gillet, M.J. Genge, G. Flynn, T. Ferroir, S. Fallon, D.S. Ebel, Z.R. Dai, P. Cordier, B. Clark, M.F. Chi, A.L. Butterworth, D.E. Brownlee, J.C. Bridges, S. Brennan, A. Brearley, J.P. Bradley, P. Bleuet, P.A. Bland, R. Bastien, Report-Mineralogy and petrology of comet 81P/Wild 2 nucleus samples. Science 314, 1735-1739 (2006b)

H.A. Zook, Spacecraft measurements of the cosmic dust flux, in Accretion of Extraterrestrial Matter Throughout Earth's History, ed. by B. Peucker-Ehrenbrink, B. Schmitz (Springer, Boston, 2001), pp. 75-92 